\title{
On the Internal Structure of a Black Hole Utilizing a 4-D Spatial Blackbody Radiation Model
}

\author{
Christopher Pilot \\ Physics Department, Gonzaga University, Spokane, WA, USA \\ Email: pilot@gonzaga.edu
}

How to cite this paper: Pilot, C. (2019) On the Internal Structure of a Black Hole Utilizing a 4-D Spatial Blackbody Radiation Model. Journal of High Energy Physics, Gravitation and Cosmology, 5, 719-772. https://doi.org/10.4236/jhepgc.2019.53039

Received: April 1, 2019

Accepted: June 18, 2019

Published: June 21, 2019

Copyright (อ 2019 by author(s) and Scientific Research Publishing Inc. This work is licensed under the Creative Commons Attribution International License (CC BY 4.0).

http://creativecommons.org/licenses/by/4.0/

\section{(c) (i) Open Access}

\begin{abstract}
A black hole is treated as a self-contained, steady state, spherically symmetric, 4-dimensional spatial ball filled with blackbody radiation, which is embedded in 3-D space. To model the interior distribution of radiation, we invoke two stellar-like equations, generalized to 4-D space, and a probability distribution function (pdf) for the actual radiative mass distribution within its interior. For our purposes, we choose a truncated Gaussian distribution, although other pdf's with support, $r \in[0, R]$, are possible. The variable, $r=r^{(4)}$, refers to the 4 -D radius within the black hole. To fix the coefficients, $(\mu, \sigma)$, associated with this distribution, we choose the mode to equal zero, which will give maximum energy density at the center of the black hole. This fixes the parameter, $\mu=0$. Our black hole does not have a singularity at the center, and, moreover, it is well-behaved within its volume. The rip or tear in the space-time continuum occurs at the event horizon, as shown in a previous work, because it is there that we transition from 3-D space to 4-D space. For the shape parameter, $\sigma$, we make use of the temperature just inside the event horizon, which is determined by the mass, or radius, of the black hole. The amount of radiative heat inflow depends on mass, or radius, and temperature, $T_{2} \geq 2.725 \mathrm{~K}$, where, $T_{2}$, is the temperature just outside the event horizon. Among the interesting consequences of this model is that the entropy, $S^{(4)}$, can be calculated as an extrinsic, versus intrinsic, variable, albeit in 4-D space. It is found that $S^{(4)}$ is much less than the comparable Bekenstein result. It also scales not as, $R^{2}$, where $R$ is the radius of the black hole. Rather, it is given by an expression involving the lower incomplete gamma function, $\gamma(s, x)$, and interestingly, scales with a more complicated function of radius. Thus, within our framework, the black hole is a highly-ordered state, in sharp
\end{abstract}


contrast to current consensus. Moreover, the model-dependent gravitational "constant" in 4-D space, $G_{r}^{(4)}$, can be determined, and this will depend on radius. For the specific pdf chosen, $G_{r}^{(4)} M_{r}=0.1 c^{2}\left(r^{4} / \sigma^{2}\right)$, where $M_{r}$ is the enclosed radiative mass of the black hole, up to, and including, radius $r$. At the event horizon, where, $r=R$, this reduces to $G_{R}^{(4)}=0.2 G R^{3} / \sigma^{2}$, due to the Schwarzschild relation between mass and radius. The quantity, $G$, is Newton's constant. There is a sharp discontinuity in gravitational strength at the 3-D/4-D interface, identified as the event horizon, which we show. The 3-D and 4-D gravitational potentials, however, can be made to match at the interface. This lines up with previous work done by the author where a discontinuity between 3-D and 4-D quantities is required in order to properly define a positive-definite radiative surface tension at the event horizon. We generalize Gauss' law in 4-D space as this will enable us to find the strength of gravity at any radius within the spherically symmetric, 4-D black hole. For the pdf chosen, $g_{r}^{(4)}=G_{r}^{(4)} M_{r} / r^{3}=0.1 c^{2} r / \sigma^{2}$, a remarkably simple and elegant result. Finally, we show that the work required to assemble the black hole against radiative pressure, which pushes out, is equal to, $0.1 M_{R} c^{2}$. This factor of 0.1 is specific to $4-\mathrm{D}$ space.

\section{Keywords}

Black Hole, 4-D Spatial Blackbody Radiation Model, Internal Structure, Radiative Pressure

\section{Introduction}

This is the second paper in two-part series on black holes, where we model a black hole as a self-contained, 4-D spatial sphere, filled with black body radiation. We focus on photonic radiation only, obeying Bose-Einstein statistics in this paper, and leave open the possibility that other forms of radiation can co-exist within the black hole, some obeying Fermi-Dirac statistics. Those other radiations will depend on temperature, and the effective number of relativistic degrees of freedom at that temperature, $g^{*}(T)$. See references [1] [2] [3] [4]. The black hole, being defined as a steady state ball of radiation in 4-D space, is embedded in 3-D space, much like a gas bubble within a liquid, or, a liquid droplet within a gas. However, the droplet is 4-dimensional, and, as we shall see, not of uniform density. There are three central issues regarding black holes for which we seek an answer. First, what is the nature of the event horizon? Second, what does the internal structure of a black hole look like? And third, can we calculate key thermodynamic variables within the black hole itself? As examples of thermodynamic quantities, what are the radiative pressure, the internal energy density, the total (radiative mass) energy density, the entropy density, and the gravitational strength within the black hole as functions of, $r$, the 4 -D radius? In the 
first paper [5], we focused on giving an answer to the first question. In this paper, we will address questions two, and three.

In a previous work [5], we presented a model to answer question one. We argued that the event horizon can be thought of as a membrane, where we have a discontinuity in space, which separates the 4-D black hole from the surrounding 3-D space. Access from one space to the other is through the event horizon, which acts as flexible membrane, and which allows for zero or net radiative heat inflow, but no net outflow. The event horizon was assumed to be infinitely thin. The temperature, $T_{1}$, just inside the event horizon in 4-D space is given strictly in terms of the black hole mass, or radius. If the temperature just outside, $T_{2}$, equals $2.725 \mathrm{~K}$, then we will assume that there is no inflow. For higher temperatures, where, $T_{2}>2.725 \mathrm{~K}$, we will assume net inflow. The amount of radiative heat inflow, $\mathrm{d} Q / \mathrm{d} t$, depends on the temperature $T_{2}$, as well as on the size, or mass, of the black hole. It can be determined using a generalized version of the Stefan-Boltzmann equation, derived in reference [5], to take into account radiative transfers between adjoining spatial dimensions. With or without net inflow of radiative heat energy, we saw that the temperature drops precipitously upon entering the black hole. This is due to the change in the dimensionality of space. Upon entering the black hole, the surface area increases dramatically, and consequently, the temperature decreases abruptly. We will show, however, in this paper, that as one reduces the 4-D radius within the black hole, the temperature will increase, layer by layer, until it reaches a maximum value at the center. At the center of the black hole, we will have a maximum temperature, and a maximum radiative energy density, for a finite volume.

The temperature just outside the event horizon must be at least $2.725 \mathrm{~K}$ to account for the $\mathrm{CMB}$ photons lying just outside, and it will be higher if there is radiative heat inflow. Other forms of matter/radiation, undoubtedly, will also have to be taken into account, such as dark matter and dark energy, but $2.725 \mathrm{~K}$ photons are the minimum blackbody contribution. We will ignore neutrino blackbody radiation, as this can be considered later, as an add-on. To define a specific inside surface temperature, $T_{1}$, we hypothesized that there is no heat inflow if the outside temperature used is the $\mathrm{CMB}$ temperature. This is, of course, a working hypothesis, which cannot be justified observationally, as far as we know. Radiative heat inflows for $T_{2}=2.725 \mathrm{~K}$ would be hard to observe, if they exist at all, as they would be very inconsequential. They would not lead to any recognizable event horizon expansion, at least not in the current cosmological epoch. Thus we cannot prove or disprove this statement; we simply accept it. By accepting this hypothesis, however, we treat the black hole as if it has the ability to adjust to outside conditions. In other words, the black hole is not a passive entity; it does interact and respond to its surroundings. We must also prevent implosion due to gravity and our pdf (probability distribution function) will guarantee that, as we shall see shortly.

For temperatures on the outside, $T_{2}$, greater than $2.725 \mathrm{~K}$, we will have net 
radiative heat inflow, $\mathrm{d} Q / \mathrm{d} t>0$. This inflow will cause the black hole to expand with time. A higher temperature on the outside will increase the rate of expansion at the event horizon. As the radius increases, the inside surface temperature, just inside the event horizon in 4-D space, however, will decrease. Moreover, there is a limit to the amount of radiative heat inflow within our framework. As shown in reference [5], the inequality, $0 \leq 1 / c \mathrm{~d} Q / \mathrm{d} t<4 F_{R}^{(3)}$, must hold, where, $F_{R}^{(3)}$, is the 3-D radiative force acting just outside the event horizon, tending to push the event horizon radially in. By definition, $F_{R}^{(3)} \equiv p_{2}^{(3)} 4 \pi R^{2}$, where $p_{2}^{(3)}$ is the radiative pressure defined in terms of temperature, $T_{2}$. This radiative pressure, being 3-dimensional, acts on surface area, $A_{R}^{(3)}=4 \pi R^{2}$. For an isolated, static black hole, where, $\mathrm{d} Q / \mathrm{d} t=0$, it was found that the temperature just on the inside surface obeys the following expression, $T_{1}=0.569 R^{-1 / 5}$. This assumes that the temperature, just outside the event horizon, has the specific value, $T_{2}=2.725 \mathrm{~K}$. For the different situation where, $T_{2}>2.725 \mathrm{~K}$, we have a dynamic black hole with net inflow, and, $\mathrm{d} Q / \mathrm{d} t>0$. In this instance, $T_{1}$, also assumes the same value as above, but as the black hole expands, its value will decrease to accommodate the increase in radius. Until the radius changes, $T_{1}$ will not change. As we shall see, fixing $T_{1}$ will determine the interior distribution of radiative mass within the black hole. This quantity is crucial. In both scenarios, $R$, is the radius of the black hole, which is related to its mass, $M_{R}$, via the well-known Schwarzschild relation, $R=2 G M_{R} / c^{2}$.

Across the 3-D/4-D membrane, we have applied a generalized version of the Stefan-Boltzmann law, which we have derived for radiative transfers between adjoining spatial dimensions. This generalized law allows for the continuous exchange of electromagnetic photons back and forth, but due to the different dimensions of space, the temperature on either side has to be different. This provides a feedback mechanism, which guarantees the stability of the event horizon. If we start with a temperature, $T_{2}=2.725 \mathrm{~K}$, on the $3-\mathrm{D}$ side, then $T_{1}=0.569 R^{-1 / 5}$ is the equilibrium temperature, just inside the event horizon, on the 4 -D side. If we have a higher temperature just outside, $T_{2}>2.725 \mathrm{~K}$, which we call a "temperature load" or "load" for short, then radiative heat will flow in. This will cause the black hole to expand, i.e., $R$ now becomes, $R=R(t)$, where $R(t)$ will start to increase. This causes the inside equilibrium temperature, $T_{1}$, to decrease. As $R$ increases to, $R^{\prime}$, the 4-D surface area, $A_{R}^{(4)}$, also increases to new value, $A_{R^{\prime}}^{(4)}$. During the process of expansion, we will see that the actual inside surface temperature, within the 4-D black hole, goes down. Not only that, but so does the core temperature, at the very center of the black hole. However the black hole will have a greater mass, greater than the one before, due to its increased size.

The event horizon is also characterized by a necessarily positive, and finite, surface tension [5]. The black hole encloses a finite, fixed volume, and so this is a requirement for positive curvature. It is, after all, a ball, but a 4-dimensional one. Having derived an expression for radiative surface tension, we were able to cal- 
culate the amount of work needed to expand the black hole against radiative forces. We came up with two expressions for the work done. The first involved the surface tension. The second made use of the coefficients of surface tension. We generalized the Young-Laplace equations for this multidimensional expansion of a membrane. Normally the Young-Laplace equations apply to 3-D objects, such as liquid droplets, or thin films, and the internal pressure is always greater than the external, outside pressure in order to guarantee a positive curvature, a convex surface. As it turns out, for a positive curvature, the 4-D radiative pressure is actually less than the $3-\mathrm{D}$ radiative pressure. This surprising result has to do with the different dimensionality of space. We were also able to show that the two expressions for the positive work done upon expansion were shown to be equivalent to one another.

Because there are two surfaces expanding, the 4-D and the 3-D surfaces, there are two contributions to the radiative work done, one assisting expansion, and the other resisting it. The overall pressure is such as to cause a resultant resisting force. The radiative surface tension, $F_{\mathrm{ST} \text { radiative }}$, was defined as, $F_{\text {ST radiative }} \equiv F_{R}^{(3)}-F_{R}^{(4)}=p_{2}^{(3)} A_{R}^{(3)}-p_{1}^{(4)} A_{R}^{(4)}$, which is always positive. All superscripts within parenthesis over a physical quantity will define the space over which that quantity is defined. Sometimes, we will dispense with this convention, as in the latter sections, when it is obvious in which space, the quantity refers to. The subscripts on the radiative pressures refer to the different temperatures, over which the pressure is calculated. The quantity, $p_{2}^{(3)}$, is calculated at temperature, $T_{2}$, just outside the event horizon in $3-\mathrm{D}$ space. The quantity, $p_{1}^{(4)}$, on the other hand, is calculated at temperature, $T_{1}$, which is the temperature just inside the event horizon, in 4-D space within the black hole. The full surface tension will consist of radiative and gravitational components. In fact, as shown in reference [5], $F_{S T}=\left(F_{\mathrm{ST} \text { radiative }}+F_{G, R}^{(4)}\right)$, where $F_{G, R}^{(4)}$ is the magnitude of the gravitational force, at $r=R$, directed radially inwards. In this paper we will derive an expression for this 4 - $\mathrm{D}$, model-dependent, gravitational force.

The radiative portion of the total surface tension was found to always obey the relation, $F_{\text {ST radiative }} \geq 0.2 F_{R}^{(3)}$, where the equality holds if there is no inflow, and the inequality applies to the situation where there is net radiative heat inflow, $\mathrm{d} Q / \mathrm{d} t>0$. As one can see, $F_{\mathrm{ST} \text { radiative }}$, is always positive and finite, in spite of the fact that the $4-\mathrm{D}$ surface area is greater than the $3-\mathrm{D}$ area. This special result of a positive-definite radiative surface tension is due to the different dimensionality of space, itself. If the black hole were a three dimensional object, then we would not have an innate radiative surface tension. The radiative surface tension at the event horizon was also found to obey the equation, $F_{\text {ST radiative }}=1 / 3 a^{(3)} 4 \pi R^{2}\left[T_{2}^{4}-4 / 5(2.725)^{4}\right]$, where $R$ is the radius of the black hole, and $T_{2}$ is the temperature load outside the event horizon. This holds, with $\left(T_{2}>2.725\right)$, or without $\left(T_{2}=2.725\right)$, net radiative heat inflow. If there is net heat inflow, radiative heat and other forms of radiative energy get transferred over to the 4-D side, and the black hole expands. Unlike a star, where the inside 
surface temperature is driven by internal heat, and inside pressure, the black hole's surface temperature is determined by external conditions. Within a star, internal factors such as nuclear fusion and production rates drive the process of expansion/contraction. We assume no energy production process going on within the black hole itself.

In this paper we focus on questions, number two, and number three. We seek to determine what the internal structure of a black hole could look like given our 4-D radiative model. Specifically, we want to see how key thermodynamic quantities can be determined, i.e. calculated. We will treat the black hole much like a star in that we will consider two stellar-like equations. These equations will be applied to radiation, once we generalize them to 4-D space. Within the black hole, we will assume a particular, steady state distribution of radiative mass. We choose a probability distribution with maximum mathematical entropy, a truncated Gaussian (=truncated Normal) pdf, which, as we shall see, will serve our purpose quite well. The radiative mass distribution is assumed to equal the total radiative energy distribution divided by the speed of light squared. Both quantities will depend on the 4-D radius within the black hole. The temperature profile within the black hole is ultimately what determines the radiative energy density. For a 4-D radiative object, having spherical symmetry, the radiative mass density, $\rho_{r}^{(4)}$, will be proportional to the temperature to the fifth power, i.e., $\rho_{r}^{(4)} \sim T_{r}^{5}$, as this is the power law that one obtains in 4-D space for blackbody radiation. The subscripts, on both, $\rho_{r}^{(4)}$, and, $T_{r}$, indicate that these quantities are radius dependent. As mentioned, the weakest temperatures are to be found just inside the event horizon. From there they increase smoothly and continuously, as one approaches the very center of the black hole.

The outline of this paper is as follows. In Section 2, we generalize two very basic 3-D stellar-like equations to 4-D space. We focus on blackbody photons but leave open the possibility that other radiations can be "packed", within this same space, some obeying Fermi-Dirac statistics. These would also apply to the massive particles trapped inside the black hole. If those particles are above freeze-out temperatures within parts of the black hole, they will be in the radiative state. Otherwise, these fermions are in the particle state, when temperatures drop below freeze-out temperatures. As will be seen, there will be layers within the black hole where temperatures are just too high for massive particles to exist; they will then have to be in the radiative mode. Other sections within the black hole can accommodate real massive particles because the temperatures are low enough.

In Section 3, we give some rationale for choosing a truncated Normal distribution to represent the mass distribution. We assume a maximum radiative mass energy density near the center, which fixes the mode to equal zero. This fixes one parameter, namely, $\mu=0$. The other parameter, the shape parameter, $\sigma$, will be determined by the inside surface temperature, $T_{1}$, just inside the event horizon, which, in turn, will depend on the size, or mass, of the black hole. As shown in reference [5], the inside and outside surface temperatures are re- 
lated to one another by the generalized Stefan-Boltzmann law. For no net radiative heat inflow, $T_{2}=2.725 \mathrm{~K}$, which is an assumption. With net radiative heat inflow, $T_{2}>2.725 \mathrm{~K}$. Either way, the inside surface temperature is given by the expression, $T_{1}=0.569 R^{-1 / 5}$. The difference is that, for an isolated, static black hole with no inflow, $R=$ constant . In the second instance with inflow, $R$ will increase as a function of time, i.e., $R=R(t)$. Thus, if we know the radius of the black hole, $R=R(t)$, we can calculate the inside surface temperature, $T_{1}$, as a function of time. Until the radius has changed, the inside surface temperature will not change. For a Gaussian distribution, which is "truncated", it is important to recognize that, $\mu$, is no longer the mean. And neither is, $\sigma$, the standard deviation. The mean and variance are complicated functions of, $R, \mu$, and, $\sigma$, in this instance.

Section 4 will concern itself with derivations. We will focus on three black holes, one having the mass of the sun. The other two will have masses ten times the mass of the sun, and, a million times that of the sun. For each, we will derive key thermodynamic variables within the black hole, as a function of radius. We will focus on black holes which are isolated and static. In other words there is no net heat inflow. The thrust of this paper is to indicate a process, and not work out all scenarios. It will be clear how to proceed if the black hole is not static, i.e., if we are dealing with a black hole having net heat inflow. Some key thermodynamic variables are 4-D mass density, $\rho_{r}^{(4)}$, and the $4-\mathrm{D}$ radiative pressure, $p_{r}^{(4)}$. We also derive expressions for the 4-D internal energy density, $u_{r}^{(4)}$, the heat density, $q_{r}^{(4)}$, and the entropy density, $s_{r}^{(4)}$. These variables will be presented in table form, where there is one table for each black hole mass. Again, we will focus on an isolated, static black hole, with no inflow, i.e. $\mathrm{d} Q / \mathrm{d} t=0$, in order to keep the discussion simple. In other papers, radiative inflow can be considered using the equations developed here, and in the previous paper. All tables are to be found, at the end of the paper.

In Section 5, we calculate certain global properties within the black hole. We find expressions for the radiative mass, $M_{r}=M_{r}^{(4)}$, from, $r=0$, up to and including radius, $r$. We also look at the work done in assembling the radiative mass against radiative pressure, $W_{r}^{(4)}$. We will calculate the gravitational constant, $G_{r}^{(4)}$, the gravitational acceleration, $g_{r}^{(4)}$, the gravitational force, $F_{G, r}^{(4)}$, and the entropy, $S_{r}^{(4)}$, among other variables, within the black hole at a particular $r$ value. The total mass, total work, and total entropy are found if we specialize to, $r=R$. At the surface we also calculate the $4-\mathrm{D}$ gravitational "constant", the gravitational force, and the gravitational potential. There will be a discontinuity in the gravitational force, but the $4-\mathrm{D}$ gravitational potential at $r=R$ can be chosen to match the $3-\mathrm{D}$ gravitational potential, by a judicious choice of integration constant. In this way we guarantee the same equipotential surface in both spaces for the same $R$ value, even though the spaces are quite different on either side. Again, three tables will be presented, one for each of the black holes considered in Section 4 . The tables list various values as a function of 
radius, in incremental steps of, $r=0,0.03 R, 0.06 R, 0.09 R$, etc. They are found at the end of this paper. Finally, in Section 6, we present our summary and conclusions. We have an Appendix, where some useful identities associated with the truncated Gaussian probability distribution function can be found, and referred to. We also give a few identities as it relates to the calculation of entropy.

\section{4-D Generalization of the Standard Hydrostatic Stellar Equations for a Black Hole}

In this section, we consider two standard stellar-like equations, and generalize them for application to our 4-D blackbody radiation model. The stellar equations, which are 3-D in character, will be generalized to 4-D space. Moreover, they will be specialized to our radiative model, which will require certain modifications.

For a star, there are four standard, 3-D stellar equations, which are typically used to model the interior of a star [6] [7]. They, in effect, determine a stars internal structure. One of these equations deals with hydrostatic equilibrium. As is well known, layer by layer, the force of gravity holds in check the radiative pressure acting radially outwards. This pressure is due to nuclear fusion processes occurring deep within the interior of the star. For a black hole, we conjecture that we also have radiative pressure, but this is not due to any kind of energy production process within its interior. Rather, we claim that we have simple packing of radiative energy, which is spherically symmetric, and 4-dimensional. This packing of radiative energy will be accomplished by way of gravity. As one heads radially outward from the center of the black hole, we have a pressure gradient, $p_{r}^{(4)}$ an internal energy density gradient, $u_{r}^{(4)}$, and a radiative heat density gradient, $q_{r}^{(4)}$, which fall off with increasing radius. Each is proportional to the fifth power of temperature, $T_{r}^{5}$, where, $T=T(r)=T_{r}$, is the temperature inside the black hole. In 4-D space the internal energy density, $u_{r}^{(4)}=a^{(4)} T_{r}^{5}$, where $a^{(4)}$ is a numerical constant equal to $a^{(4)}=4.7481 \times 10^{-13} \mathrm{~J} \cdot \mathrm{m}^{-4} \cdot \mathrm{K}^{5}$. Refer to reference [5], where it is also shown that, $p_{r}^{(4)}=1 / 4 u_{r}^{(4)}$, and, $q_{r}^{(4)}=5 / 4 u_{r}^{(4)}$. MKS units will be used throughout this paper, even when not explicitly written out.

In a very real sense, our 4-D model for radiative energy trapped within a spherical surface is much like a capacitor. This capacitor is rather exotic in that it is four dimensional. It will also not have a uniform distribution of radiative energy within its confines. The size of this capacitor will determine how much radiative mass it will be able to store (and vice versa). And the radiative mass density at a particular 4-D radius will determine its permittivity. The permittivity will be a function of radius, as radiative mass density within the black hole is a function of radius. A capacitor is a passive object in that no energy production occurs within its volume. It simply stores energy, and therefore has to be "charged" in some sense. Massive and massless energy inflow into the black hole will serve this function. We shall allude to this analogy on occasion, as this is a 
good way of imagining our version of a black hole.

Since the black hole has a finite radius in 4-D space, which corresponds to the Schwarzschild radius in $3-\mathrm{D}$ space, it has support, $r \in[0, R]$. In other words, only radii within this realm can be considered, where the square brackets signify that we have to include radius, $r=0$, as well as, $r=R$. The temperature, the radiative pressure, the internal energy density, etc. within the black hole are also defined within the same realm of support. There is no probability of finding these entities outside the event horizon.

Coming back to $3-\mathrm{D}$ space, as mentioned, there are typically 4 equations that one looks at for modeling the interior of a star. Two of these fundamental equations are:

$$
\mathrm{d} M_{r}^{(3)} / \mathrm{d} r=4 \pi r^{2} \rho_{r}^{(3)} \quad \text { (mass conservation) }
$$

and,

$$
\mathrm{d} p_{r}^{(3)} / \mathrm{d} r=-G M_{r}^{(3)} \rho_{r}^{(3)} / r \quad \text { (hydrostatic equilibrium) (2-2) }
$$

In Equation, (2-1), $\mathrm{d} M_{r}^{(3)}$, is the 3-D increase in radiative mass for a corresponding increase in radius, from radius, $r$, to radius, $r+\mathrm{d} r$. The quantity, $\rho_{r}^{(3)}$, is the radiative mass density at radius, $r$, between $r$ and $r+\mathrm{d} r$. In Equation, $(2-2), \quad \mathrm{d} p_{r}^{(3)}$, is the three dimensional increase in radiative pressure for a corresponding increase in radius, $r$, from $r$ to $r+\mathrm{d} r$. Since the right hand side is negative, we actually have a decrease in pressure for an increase in radius, starting from the center of a star. On the right hand side of Equation, (2-2), we have Newton's constant, $G$. We also have, $M_{r}^{(3)}$, which is the radiative mass, enclosed within a volume of radius, $r$. Equation, (2-2), in effect, tells us that the radiative pressure pushing the layer out at radius, $r$, is counterbalanced by the gravitational force, pulling that very same layer in.

There are two other equations typically considered in conjunction with these Equations, (2-1), and, (2-2), for modeling the interior of a star. They are not relevant here. First, there is no energy production assumed within the black hole, and hence the nuclear production rate, sometimes designated as, $\varepsilon_{r}^{(3)}$, in Watts/kg is zero. Moreover, there is no assumed energy transport within the black hole. We have no diffusivity, i.e., no scattering and/or absorption of photons passing through a medium. Even though the diffusivity coefficient, $\kappa_{r}^{(3)}$, is zero, there will, however, be a temperature gradient, which is non-trivial. In other words, $\mathrm{d} T_{r}^{(4)} / \mathrm{d} r \neq 0$. This will be due to simple packing of radiant energy within the black hole. The pdf, which is chosen, will tell us how to pack the radiative mass, and gravity will ensure that the stored energy remains stable. Again, in a very real sense, the black hole will function much like a passive 4 -D capacitor, and one that can be loaded, or "charged" with extra mass/energy.

It is our hypothesis and contention that a black hole is a self-contained, spherically symmetric object filled with blackbody radiation. We therefore generalize the two equations listed above, Equations, (2-1), and, (2-2), to 4-D space. We take each in turn, and start with Equation, (2-1). The 4-D generalization is 


$$
\mathrm{d} M_{r}^{(4)} / \mathrm{d} r=2 \pi^{2} r^{3} \rho_{r}^{(4)} \quad \text { (mass conservation) (2-3) }
$$

here, $\rho_{r}^{(4)}$, is the 4-D mass density defined at radius, $r$, in $\mathrm{kg} / \mathrm{m}^{4}$, and, $2 \pi^{2} r^{3}$ is the corresponding 4-D surface area, $A_{r}^{(4)}=2 \pi^{2} r^{3}$, at radius, $r$. The quantity, $\mathrm{d} M_{r}^{(4)}$, is the infinitesimal increase in radiative mass when we increase $r$, from radius, $r$, to radius, $r+\mathrm{d} r$. The 4 -D mass density, $\rho_{r}^{(4)}$, at radius $r$, will be proportional to $T_{r}^{5}$, where, $T_{r}$ is the temperature at that radius. We can integrate Equation, (2-3), to find the mass, $M_{r}^{(4)}$, up to and including $r$. The result is

$$
M_{r}^{(4)}=\int_{0}^{r} \mathrm{~d} M_{r}^{(4)}=\int_{0}^{r} \rho_{r}^{(4)} A_{r}^{(4)} \mathrm{d} r
$$

here, $A_{r}^{(4)}=2 \pi^{2} r^{3}$ is the 4-D surface area. The maximum value for mass is obtained when the radius, $r$, equals, $R$. The radius, $R$, characterizes the event horizon. Inserting this as our limit of integration gives

$$
M_{R}^{(4)}=\int_{0}^{R} \rho_{r}^{(4)} A_{r}^{(4)} \mathrm{d} r
$$

This is our expression for the total radiative mass associated with a 4-D black hole, which is the same mass we perceive in 3-D space.

Now, we know that the mass of a black hole is proportional to its radius because of the Schwarzschild relation, $R=2 G M_{R} / c^{2}$. Comparing this to Equation, (2-5), it would appear that, $\rho_{r}^{(4)} A_{r}^{(4)}$, is a constant, i.e.,

$$
\rho_{r}^{(4)} A_{r}^{(4)}=c^{2} /(2 G)
$$

If we substitute this expression into Equation, (2-5), then Equation, (2-5), would be trivially satisfied. However, as we shall see shortly, this will not allow for any gravitational force to act within the black hole. Although this solution would allow us to "pack in" enough radiative mass to make up the mass of the black hole, it would come at a cost. The cost is that the temperature at the inside surface would then be excessive. Therefore, we will have to dismiss this solution.

We propose a more general solution of the form,

$$
\rho_{r}^{(4)} A_{r}^{(4)}=f_{r} M_{R}
$$

In this expression, $f_{r}$, is our probability distribution function (pdf), satisfying the condition,

$$
\int_{0}^{R} f_{r} \mathrm{~d} r=1
$$

The specific choice of function, $f_{r}$, will be postponed until Section 3. For now, $f_{r}$, has units of meters ${ }^{-1}$, and the probability of finding the radiative mass between $r_{1}$ and $r_{2}$, is given by the following expression, defined in 4-D space,

$$
\operatorname{Probl}\left(r_{1} \leq r \leq r_{2}\right)=\int_{r_{1}}^{r_{2}} f_{r} \mathrm{~d} r
$$

The pdf, defined by Equation, (2-8), is truncated, and has support, $r \in[0, R]$; outside of this range in radius, $r$, there is no probability of finding 4-D radiative mass. 
We next integrate Equation, (2-7), taking into account, Equation (2-8). This gives

$$
M_{R}=\int_{0}^{R} f_{r} M_{R} \mathrm{~d} r=M_{R} \int_{0}^{R} f_{r} \mathrm{~d} r
$$

This is, of course, an identity due to Equation, (2-8). And so, more generally,

$$
M_{r}=\int_{0}^{r} f_{r} M_{R} \mathrm{~d} r=M_{R} \int_{0}^{r} f_{r} \mathrm{~d} r=M_{R} F_{r}^{C D F}
$$

In the second line, we have introduced the cumulative probability distribution function, $F_{r}^{C D F}$, which is defined by the Equation

$$
F_{r}^{C D F} \equiv \int_{0}^{r} f_{r} \mathrm{~d} r
$$

This is the sum of all probabilities, from radius, $r=0$, up to, and including, radius, $r$. By definition, if the upper bound radius is reached, then, $r=R$, and, $F_{R}^{C D F}=1$. The probability of finding the radiative mass between, $r=0$, and, $r=R$, is with certainty, or $100 \%$, which equals one numerically.

There are many pdf's, and corresponding cumulative probability distribution functions (cdf's), which will satisfy Equations, (2-8), (2-9), and, (2-10). Equation, (2-6), is but one choice. This can be seen as follows. Another way to write the right hand side of Equation, (2-6), is $M_{R} / R$, because of the Schwarzschild relation. Therefore, upon comparing with Equation, (2-7), this would give,

$$
f_{r}=1 / R=\text { constant } \quad \text { (truncated uniform distribution) }(2-1
$$

This depends solely on the size, or mass, of the black hole. In this instance, we obtain a truncated uniform distribution, where the probability distribution function is everywhere the same within the black hole. That would mean that, $\rho_{r}^{(4)} A_{r}^{(4)}=$ constant, throughout the black hole. As we shall see, this is not a very interesting solution. A much better choice for pdf will be a truncated Gaussian distribution function, to be introduced in Section 3.

We now come back to Equation, (2-2). The 4-D generalization would read

$$
\mathrm{d} p_{r}^{(4)} / \mathrm{d} r=-G_{r}^{(4)} M_{r}^{(4)} \rho_{r}^{(4)} / r^{3}
$$

In Equation, (2-14), the quantity, $\mathrm{d} p_{r}^{(4)}$, is the increase in $4-\mathrm{D}$ radiative pressure, in going from radius, $r$, to radius, $r+\mathrm{d} r$. The mass, $M_{r}^{(4)}$, is the radiative mass enclosed, between $r=0$, and, radius, $r$. The quantity, $G_{r}^{(4)}$, is the 4-D analogue of Newton's constant. It has a value, which is model dependent, i.e., specifically depends of the pdf chosen. We will soon derive an expression for it, and we will notice that it will depend on radius. The MKS unit for mass density in this 4-D world is, $\mathrm{kg} / \mathrm{m}^{4}$. The MKS unit for the 4-D gravitational "constant", $G_{r}^{(4)}$, is, Newtons ${ }^{*} \mathrm{~m}^{3} / \mathrm{kg}^{2}$. It can be easily verified that Equation, (2-14), is dimensionally consistent, even though the units apply to a $4-\mathrm{D}$ world.

Equation, (2-14), however, is not quite correct. It is missing a term on the right hand side, which we will now correct for. To show this, we first need to develop some general ideas. We start with the internal energy density. As is known [8] [9] [10] [11] [12], in N-dimensional space, the internal energy density, 
$u^{(N)}$, can be written as

$u^{(N)}=u(N, T)=2(N-1) \pi^{N / 2}\left(k_{B} T\right)^{N+1} \zeta(N+1) \Gamma(N+1) /\left[(h c)^{N} \Gamma(N / 2)\right]$

In this equation, $k_{B}$ is Boltzmann's constant, $c$ equals the speed of light, $h$ is Planck's constant, $\zeta(x)$ is the zeta function, and $\Gamma(x)$ is the gamma function. Moreover, the radiative pressure, heat density, and the entropy density, $s^{(N)}$, are related to $u^{(N)}$ via the equations,

$$
p^{(N)}=u^{(N)} / N, q^{(N)}=(N+1) u^{(N)} / N, s^{(N)}=q^{(N)} / T
$$

These relations depend only on the dimensionality of space, $N$, and the temperature, $T$, because $u^{(N)}$ is, in itself, a function of temperature and dimension. In 4-D space, Equation, (2-15), reduces to

$$
u_{r}^{(4)}=u^{(4)}(r)=a^{(4)} T_{r}^{5}=3 \pi / 2 c \sigma^{(4)} T_{r}^{5}=4.7481 \times 10^{-13} T_{r}^{5}
$$

In Equation, (2-17), $\sigma^{(4)}$ is the Stefan-Boltzmann constant, generalized to 4-D space. From Equations, (2-16), it follows that

$$
p^{(4)}=u^{(4)} / 4, q^{(4)}=(5 / 4) u^{(4)}, s^{(4)}=q^{(4)} / T
$$

The particular factors, which relate pressure to internal energy density, radiative heat density to internal energy density, etc. depend on the dimension of space chosen, which in this instance equals four.

Moreover, it is possible to define a total energy "density", $e_{r}^{(4)}$, by the relation,

$$
\mathrm{d} E_{r}^{(4)}=u_{r}^{(4)} \mathrm{d} V_{r}^{(4)}+p_{r}^{(4)} \mathrm{d} V_{r}^{(4)}+q_{r}^{(4)} \mathrm{d} V_{r}^{(4)} \equiv e_{r}^{(4)} \mathrm{d} V_{r}^{(4)}
$$

By definition, the total radiative energy "density" is found by using

$$
e_{r}^{(4)} \equiv u_{r}^{(4)}+p_{r}^{(4)}+q_{r}^{(4)}
$$

In these equations, $u_{r}^{(4)}$, is the $4-\mathrm{D}$ internal energy density, $p_{r}^{(4)}$, the $4-\mathrm{D}$ radiative pressure, and $q_{r}^{(4)}$, the 4 -D heat density, all defined at a particular radius, $r$, in 4 -D space. The quantities are radius dependent because the temperature depends on radius. Hence we have the subscripts, $r$. Being 4-D quantities, the densities and pressures have different units than those of their 3-D counterparts.

In Equation, (2-19), $\mathrm{d} E_{r}^{(4)}$ is the total energy trapped in a layer between radius, $r$, and radius, $r+\mathrm{d} r$. The volume element, $\mathrm{d} V_{r}^{(4)}=A_{r}^{(4)} \mathrm{d} r$, is the corresponding, $4-\mathrm{D}$, infinitesimal volume element. In $4-\mathrm{D}$, the surface area, $A_{r}^{(4)}=2 \pi^{2} r^{3}$.

Technically, Equation, (2-20), only makes sense when multiplied by a volume element, or a surface area. This is because the units for radiative pressure (Newtons $/ \mathrm{m}^{3}$ ) are not the same as those for internal energy density, or heat energy density, (Joules $/ \mathrm{m}^{4}$ ). Both terms are represented on the right hand side of "Equation", (2-20). However, when multiplied by a volume element, or a surface area, we retrieve units which do match, as in Equation, (2-19). When we write an expression such as relations, (2-20), or, (2-18a), we implicitly assume that it is to be multiplied by such terms in order to make it dimensionally valid.

The total radiative energy density of blackbody photons, in 4-D space, is 
represented by the sum given in relation, (2-20). It consists of three components, internal energy density, plus heat energy density, plus radiative pressure. All are defined at the same particular temperature. This is the temperature within a specific layer within the black hole. Being defined at the same temperature, all three blackbody contributions exist simultaneously. In other words, it is an all or nothing situation, because of our relations, (2-18).

Furthermore, we assume that all three components, indicated in Equation, (2-20), make up the radiative mass within a particular layer inside the black hole. In other words, $e_{r}^{(4)}=\rho_{r}^{(4)} c^{2}$, where $\rho_{r}^{(4)}$ is the radiative mass density, in $\mathrm{kg} / \mathrm{m}^{4}$, at radius, $r$, between radii, $r$ and $r+\mathrm{d} r$. This gives,

$$
\rho_{r}^{(4)} A_{r}^{(4)}=e_{r}^{(4)} / c^{2} A_{r}^{(4)}=10 / 4 u_{r}^{(4)} / c^{2} A_{r}^{(4)}=10 p_{r}^{(4)} / c^{2} A_{r}^{(4)}
$$

For the second and third lines in Equation, (2-21), we have used the relations, (2-20), and, (2-18). It can be further demonstrated that,

$$
e_{r}^{(4)}=10 p_{r}^{(4)}=2 q_{r}^{(4)}=10 / 4 u_{r}^{(4)}
$$

The factors sitting out in front are specific to 4-D space. For this relation, (2-22), to make sense in terms of units matching, it is assumed that we multiply this equation by a volume, or surface element. It is under this condition, that an "equation" such as relation, (2-22), holds valid. The radiative heat energy density, $q_{r}^{(4)}$, is related to the entropy density, $s_{r}^{(4)}$, by means of the last Equation in (2-18), which for a particular radius, reads, $s_{r}^{(4)}=q_{r}^{(4)} / T_{r}$. We treat the entropy density as a state variable, which depends on the temperature within an infinitesimal layer, at a particular radius $r$.

Next, we recognize that, if we focus on a layer within the black hole, at radius, $r$, there is a 4 -D radiative force, $F_{r}^{(4)}$. This force is directed radially out, if viewed from above, and radially in, if viewed from below. By definition,

$$
F_{r}^{(4)} \equiv p_{r}^{(4)} A_{r}^{(4)}=p_{r}^{(4)} 2 \pi^{2} r^{3}
$$

It will turn out that if a layer has a thickness, $\mathrm{d} r$, between $r$ and $r+\mathrm{d} r$, there will be a greater radiative force pushing out, at $r$, than pushing in at $r+\mathrm{d} r$. This will be due to the fact that the temperature closer in to the center, will always be greater than the temperature slightly further out. We can also write, because of Equation, (2-22),

$$
e_{r}^{(4)} A_{r}^{(4)}=10 p_{r}^{(4)} A_{r}^{(4)}=10 F_{r}^{(4)}
$$

Therefore, from Equation, (2-21), it follows that

$$
\rho_{r}^{(4)} A_{r}^{(4)}=10 F_{r}^{(4)} / c^{2}=f_{r} M_{R}
$$

Equation, (2-7), has also been utilized. Relation, (2-25), is a fundamental equation.

From the last equality, in Equations, (2-25), we see very clearly, that the chosen pdf, $f_{r}$, will determine the radiative mass distribution, as well as the radiative force distribution, within the black hole. It will also determine, the distribution of internal energy density, and of radiative heat density. From Equation, 
(2-22), we notice that $u_{r}^{(4)} A_{r}^{(4)}, p_{r}^{(4)} A_{r}^{(4)}, q_{r}^{(4)} A_{r}^{(4)}$, are all proportional to $e_{r}^{(4)} A_{r}^{(4)}$. This, in turn, is proportional to, $\rho_{r}^{(4)} A_{r}^{(4)}$, by Equation, (2-21). And finally, $\rho_{r}^{(4)} A_{r}^{(4)}$, is proportional to $F_{r}^{(4)}$, and $f_{r}$, by Equation, (2-25). We see from all this that the pdf chosen will ultimately determine these other quantities as well. The units for, $u_{r}^{(4)} A_{r}^{(4)}, p_{r}^{(4)} A_{r}^{(4)}, q_{r}^{(4)} A_{r}^{(4)}$, and $e_{r}^{(4)} A_{r}^{(4)}$, are Joules/m. Those for, $\rho_{r}^{(4)} A_{r}^{(4)}$, are, $\mathrm{kg} / \mathrm{m}$. Summarizing, the pdf, $f_{r}$, will determine not only how radiative mass is packed within the black hole, but also, how the various forms of radiative energy density and radiative forces are distributed within the 4-D volume. Equation, (2-25), essentially tells us how the black hole is structured within its interior. Ultimately, it will rest upon our choice of pdf, i.e., the specific, $f_{r}$, function chosen to model the interior.

We now come back to our original Equation, (2-14). Consider a specific layer, between radii, $r$, and, $r+\mathrm{d} r$, within the 4 -D black hole. The forces acting on this layer are as follows:

$$
F_{r}^{(4)}-F_{r+\mathrm{d} r}^{(4)}=\left|\mathrm{d} F_{G, r}^{(4)}\right|
$$

In this expression, $F_{r}^{(4)}$, is the $4-\mathrm{D}$ radiative force pushing radially out at radius, $r$, and $F_{r+\mathrm{d} r}^{(4)}$ is the 4-D radiative force pushing the layer radially in at radius, $r+\mathrm{d} r$, from above. The temperature at radius, $r$, is slightly (infinitesimally) larger than that at radius, $r+\mathrm{d} r$, and so we have a slight difference in radiative pressure. We also have a slight difference due to the larger surface area at radius, $r+\mathrm{d} r$, versus that at radius, $r$. On the right hand side of Equation, (2-26), we have the infinitesimal gravitational force acting on this layer between, $r$, and, $r+\mathrm{d} r$. This is acting on the radiative mass trapped within that layer, and this force is directly radially inwards. We will prove in Section 5, due to Gauss's law in $4-\mathrm{D}$ space, that

$$
\begin{aligned}
\left|\mathrm{d} F_{G, r}^{(4)}\right| & =G_{r}^{(4)} M_{r}^{(4)} \mathrm{d} M_{r}^{(4)} / r^{3} \\
& =G_{r}^{(4)} M_{r}^{(4)} \rho_{r}^{(4)} A_{r}^{(4)} \mathrm{d} r / r^{3} \\
& =G_{r}^{(4)} M_{r}^{(4)} \rho_{r}^{(4)} 2 \pi^{2} \mathrm{~d} r
\end{aligned}
$$

The infinitesimal gravitational force depends on the strength of gravity, the enclosed radiative mass, $M_{r}^{(4)}$, and the radiative mass density at radius, $r$. This is the force acting on the layer of thickness, $\mathrm{d} r$, out at a specific distance, $r$, from the center of the black hole.

We next rewrite Equation, (2-26), as follows:

$$
\begin{aligned}
\left|\mathrm{d} F_{G, r}^{(4)}\right| & =F_{r}^{(4)}-F_{r+\mathrm{d} r}^{(4)}=F_{r}^{(4)}-\left(F_{r}^{(4)}+\mathrm{d} F_{r}^{(4)}\right)=-\mathrm{d} F_{r}^{(4)} \\
& =-\mathrm{d}\left(p_{r}^{(4)} A_{r}^{(4)}\right)=-\mathrm{d} p_{r}^{(4)} A_{r}^{(4)}-p_{r}^{(4)} \mathrm{d} A_{r}^{(4)} \\
& =-\mathrm{d} p_{r}^{(4)} A_{r}^{(4)}-p_{r}^{(4)} 6 \pi^{2} r^{2} \mathrm{~d} r
\end{aligned}
$$

In the last line, we used the 4-D surface area, $A_{r}^{(4)}=2 \pi^{2} r^{3}$, to calculate, $\mathrm{d} A_{r}^{(4)}=6 \pi^{2} r^{2} \mathrm{~d} r$. From the $4^{\text {th }}$ line, we notice that if, $p_{r}^{(4)} A_{r}^{(4)}=$ constant, then there can be no gravitational force within the black hole. The left hand side 
would then equal zero. This allowed us to disqualify Equation, (2-6), as a solution. By Equation, (2-21), $\rho_{r}^{(4)} A_{r}^{(4)}$ is proportional to $p_{r}^{(4)} A_{r}^{(4)}$. Thus, if one expression, is a constant, then, so too, is the other expression. We also see that Equation, (2-13), is inadmissible, because, if it were, then by Equation, (2-7), $\rho_{r}^{(4)} A_{r}^{(4)}$ would be proportional to a constant. This, in turn, would make the right hand side of the fourth line, in Equations, (2-28), equal to zero. To make a long story short, a uniform probability distribution function is not feasible. Both Equations, (2-6), and, (2-13), refer to this scenario. A uniform distribution is too simplistic to model the interior of a black hole. It would also lead to much too high inner-surface temperatures for a black hole, if we want to pack the required mass within its confines.

We next substitute the right hand side of Equation, (2-27), into the left hand side of relation, (2-28), since these are equivalent. We thereby obtain

$$
G_{r}^{(4)} M_{r}^{(4)} \rho_{r}^{(4)} 2 \pi^{2} \mathrm{~d} r=-\mathrm{d} p_{r}^{(4)} A_{r}^{(4)}-p_{r}^{(4)} 6 \pi^{2} r^{2} \mathrm{~d} r
$$

This we divide by, $\mathrm{d} r$, and rearrange terms. The result is

$$
\mathrm{d} p_{r}^{(4)} / \mathrm{d} r A_{r}^{(4)}=-G_{r}^{(4)} M_{r}^{(4)} \rho_{r}^{(4)} 2 \pi^{2}-p_{r}^{(4)} 6 \pi^{2} r^{2}
$$

or, dividing by 4 -D surface area,

$$
\mathrm{d} p_{r}^{(4)} / \mathrm{d} r=-G_{r}^{(4)} M_{r}^{(4)} \rho_{r}^{(4)} / r^{3}-3 p_{r}^{(4)} / r
$$

We can compare this to our original ansatz, Equation, (2-14). Right away, we notice that the second term in Equation, (2-30), is new! Equation, (2-14), is missing this term, and cannot be correct because it does not take into account the change in surface area. Equation, (2-14), assumes that, $A_{r}^{(4)}=A_{r+d r}^{(4)}$, which is patently false. If we retrace our steps, we recognize that $p_{r+\mathrm{d} r}^{(4)}$ acts radially inwards, at radius, $r+\mathrm{d} r$, and it acts on surface area, $A_{r+\mathrm{d} r}^{(4)}$. The radiative pressure, $p_{r}^{(4)}$, on the other hand, is directed radially out, and it acts on a different surface area, $A_{r}^{(4)}$. The difference in radiative forces in Equation, (2-28), requires us to take into account not only a change in pressure, but also, at the same time, the change in areas, upon which these pressures are acting. In the $4^{\text {th }}$ and $5^{\text {th }}$ lines of Equations, (2-28), we see this very explicitly. The change in area is what leads to the second term on the right hand side, in the last line of Equation, (2-28). In 3-D stellar hydrostatics, apparently, the second term must be negligible when compared to the first term, and for this reason, it is probably ignored. We do not do so here. That second term on the right hand side of Equation, (2-30), is crucial.

\section{Choosing a Truncated Gaussian as a PDF for a Radiative Mass Distribution within the Black Hole; Determining the Shape Parameter}

As was shown in Section 2, Equations, (2-6), and, (2-13), led to a naïve solution for determining the distribution of radiative mass within the black hole. If the distribution of radiative mass within the black hole were uniform, then a gravi- 
tational force within its interior cannot be accommodated. Nor would we be able to keep the inner surface temperature small with such a choice, and still pack the requisite mass within radius, $R$. We are thus led to consider other pdfs which will bypass these problems. One good choice for our purposes is a truncated Gaussian [13]-[18]. In this section, we will fix the parameters associated with this distribution, such that we can model the interior for any size black hole. In this section, and the remaining sections, we will typically drop the superscript (4) on variables as a matter of convenience, as all quantities are defined in 4-D space.

First and foremost, we should recognize that other choices [19] [20] [21] [22] for a continuous truncated pdf are possible. They would lead, like the truncated Gaussian we are about to choose, to steep increases in radiative densities and pressures within the interior. Among the possibilities, we could list the truncated exponential distribution, the beta distribution, the Kumaraswamy distribution, etc. These all have support, $r \in[0, R]$, and satisfy Equations, (2-8), (2-9) and (2-12). Moreover, upon a judicious choice of parameters, these probability distributions can mimic the truncated Gaussian in many ways. The exponential involves only one parameter; the other two listed, require two parameters in order to specify the distribution. We will however choose the Gaussian or Normal distribution, $f_{r}=f(r, \mu, \sigma ; a, b)=f(r, \mu, \sigma ; 0, R)$, where $(\mu, \sigma)$ are the parameters to be determined, and $[a, b]=[0, R]$ are the lower and upper bounds for the $r=$ radius value.

The truncated Gaussian, or truncated Normal, distribution with parameters, $(\mu, \sigma)$, will serve our purposes well for the following reasons. We list them in bullet form.

1) The pdf is relatively simple, somewhat familiar, and non-trivial

2) Being a continuous distribution, this pdf can be made to smoothly increase, starting from a minimum value at, $r=R$, to a maximum value at, $r=0$.

3) This pdf becomes particularly simple if the mode is chosen at, $r=0$. In this situation, $\mu=0$, and our two parameters reduce effectively to one, the shape parameter, $\sigma$.

4) This pdf can be made to pack an incredible mass within a relatively small volume, especially in 4-D space, and still keep the inside surface temperature very low.

5) The pdf has maximum entropy of all the continuous distributions. In fact, random error follows this kind of distribution. It seems, in this regard, to be an efficient way to pack radiation energy. We have a distribution of blackbody energy based on temperature called Planck's law. Superimposed on this is a distribution based on radius, our truncated Gaussian.

For these reasons, we pursue this choice. It may turn out, however, that other truncated pdf's have more desirable characteristics and behavior. This can be worked out in other papers. We emphasize that all properties within the black hole in this paper are pdf-model dependent. This includes the calculation of en- 
tropy, gravitational constant, gravitational force, etc. and so the choice is very important. Great flexibility is present, however, for working out various scenarios, with this, and other probability distribution functions.

We will assume a-priori, that the mode is to be set at, $r=0$. As mentioned several times, this fixes the parameter $\mu$. Under this assumption, $\mu=0$, and our pdf, $f_{r}$, takes the following, much-simplified form,

$$
f_{r}=f(r, 0, \sigma ; 0, R)=\sqrt{(2 / \pi)} 1 / \sigma \exp \left(-r^{2} /\left(2 \sigma^{2}\right)\right) 1 / \operatorname{erf}(R /(\sqrt{2} \sigma))
$$

Thus, the maximum radiative energy density is at, $r=0$, the very center of the black hole. In this equation, $\operatorname{erf}(x)$ is the error function, and $\sigma$ is the shape parameter. The corresponding cumulative distribution function, $F_{r}^{C D F}$, is found using the defining relation, (2-12). The result of this integration is,

$$
F_{r}^{C D F}=F(r, 0, \sigma ; 0, R)=\operatorname{erf}(r / \sqrt{2} \sigma) / \operatorname{erf}(R / \sqrt{2} \sigma)
$$

This leads trivially to, $F_{R}^{C D F}=1$. See Equations, (2-12), and, (2-8). In the special case where, $\mu=0$, the mean is given by the following, much-simplified, function,

$$
\text { Mean }=\sqrt{(2 / \pi)} \sigma\left[1-\exp \left(-R^{2} /\left(2 \sigma^{2}\right)\right)\right] 1 / \operatorname{erf}(R / \sqrt{2} \sigma)
$$

The variance can also be calculated. The result is

$$
\begin{aligned}
\text { Var }= & \sigma^{2}[1-R / \sigma[1+\operatorname{erf}(R / \sqrt{2} \sigma)] / \operatorname{erf}(R / \sqrt{2} \sigma)] \\
& -\frac{2}{\pi}\left[1-\exp \left(-R^{2} /\left(2 \sigma^{2}\right)\right) / \operatorname{erf}(R / \sqrt{2} \sigma)\right]^{2}
\end{aligned}
$$

Other higher moments can be calculated. We note that for the truncated Gaussian, the mean is not simply equal to, $\mu$. Neither is the standard deviation, equal to, $\sigma$. The mean is calculated using Equation, (3-3). We see that its value depends on $\sigma$, the shape parameter, and radius, $R$. The standard deviation is given by the expression, $S T D=\sqrt{V a r}$, where the variance is written out in Equation, (3-4).

We know that Equation, (2-7), is a requirement. We can specialize this equation to zero radius, and, $r=R$. In the first instance, we obtain, with the help of Equation, (3-1),

$$
\rho_{0} A_{0}=M_{R} f_{0}=M_{R} \sqrt{(2 / \pi)} 1 / \sigma 1 / \operatorname{erf}(R / \sqrt{2} \sigma)
$$

In the second instance, we find, again with the help of Equation, (3-1),

$$
\rho_{R} A_{R}=M_{R} f_{R}=M_{R} \sqrt{(2 / \pi)} 1 / \sigma \exp \left(-R^{2} / 2 \sigma^{2}\right) / \operatorname{erf}(R / \sqrt{2} \sigma)
$$

The superscripts have been dropped as we are dealing here exclusively with 4-D quantities.

Equation, (3-5), is to be understood, in the following sense. In the limit where, $r \rightarrow 0$, this equality holds. A density function such as, $\rho_{0}$, requires a finite volume, and so, on the left hand side of Equation, (3-5), we require a minimum volume. The right hand side is definitely not volume dependent, and unequal to 
zero. For left and right hand sides to match, we imagine a limiting process where the right hand side is always finite, but the left hand side approaches, but never reaches, $r=0$. It is in this limit that we are to understand Equation, (3-5). We also notice that the right hand side of Equation, (3-6), is unequal to zero (unless $\sigma=\infty)$, and thus finite in value.

We next divide the left hand side of Equation, (3-5), by the left hand side of Equation, (3-6). We do the same thing on the right hand side. Performing this operation lets us write

$$
\rho_{0} A_{0} /\left(\rho_{R} A_{R}\right)=f_{0} / f_{R}=\exp \left(R^{2} / 2 \sigma^{2}\right)
$$

We shall see, shortly, that the quantity, $R^{2} / 2 \sigma^{2}$, is, in practice, already large when applied to black holes. Thus the ratio, $f_{0} / f_{R}$, is dramatically large.

More generally, following the steps above, if the radius lies within the range, $0 \leq r \leq R$, we can use Equations, (2-7), and, (3-1), to demonstrate that

$$
\rho_{r} A_{r} /\left(\rho_{R} A_{R}\right)=f_{r} / f_{R}=\exp \left[\left(R^{2}-r^{2}\right) / 2 \sigma^{2}\right]
$$

With this equation, we see that the product, $\rho_{r} A_{r}$, is monotonically decreasing with increasing radius. We have a maximum value for linear radiative mass density, $\rho_{r} A_{r}$, at, $r=0$, and this steadily decreases, until we achieve a minimum at $r=R$. Not only is radiative mass density, $\rho_{r} A_{r}$, decreasing with increasing $r$, but so too are, the internal energy density, $u_{r} A_{r}$, the radiative force, $p_{r} A_{r}=F_{r}=F_{r}^{(4)}$, and the radiative heat density, $q_{r} A_{r}$. Refer to Equations, $(2-21),(2-22),(2-23)$, and, (2-24), where these specific proportionalities are established. These quantities, must therefore, also decrease according to Equation, (3-8), for increasing radius. It remains to determine the parameter, $\sigma$, and this we do next.

One way to find the shape parameter, $\sigma$, is to start with Equation, (2-25). We rewrite it here in the form,

$$
f_{r} M_{R}=10 F_{r} / c^{2}
$$

On the left hand side, we have the pdf, whereas on the right hand side we have the 4 -D radiative force, at radius, $r$, within the black hole. We specialize Equation, (3-9), to radius, $r=R$. So we are just inside the event horizon, on the $4-\mathrm{D}$ side. This gives

$$
f_{R} M_{R}=10 F_{R} / c^{2}
$$

We next recognize that for an isolated, static black hole (ISBH), with no heat inflow,

$$
5 F_{R}^{(4)}=4 F_{R}^{(3)}
$$

This was proven in reference [5], using the generalized Stefan-Boltzmann law at the 3-D/4-D interface. Substituting this expression into the right hand side of Equation, (3-10), renders

$$
f_{R} M_{R}=8 F_{R}^{(3)} / c^{2}
$$


The quantity, $F_{R}^{(3)}$, is the radiative force right outside the event horizon, on the 3-D side. For an isolated, static black hole, it was assumed that the radiative temperature just outside the event horizon is equal to, $2.725 \mathrm{~K}$, the CMB temperature. Thus, $F_{R}^{(3)}$, can easily be determined numerically. The result is

$$
\begin{aligned}
F_{R}^{(3)} & =p_{R}^{(3)} A_{R}^{(3)}=1 / 3 u_{R}^{(3)} 4 \pi R^{2} \\
& =1 / 3 a^{(3)}(2.725)^{4} 4 \pi R^{2} \\
& =1 / 3\left(7.5657 \times 10^{-16}\right)(2.725)^{4} 4 \pi R^{2} \\
& =1.747 \times 10^{-13} R^{2}
\end{aligned}
$$

In the first line, we made use of the first equality in Equations, (2-16). For the $2^{\text {nd }}$ and $3^{\text {rd }}$ lines, it is well-known that, in 3-D space, the internal energy density can be expressed as, $u_{R}^{(3)}=a^{(3)} T^{4}=\left(7.5657 \times 10^{-16}\right) T^{4}$, in MKS units.

We next substitute the right hand side of Equation, (3-13), into the right hand side of Equation, (3-12), in order to eliminate 3-D radiative force, $F_{R}^{(3)}$. Bringing over the factor of total radiative mass, $M_{R}$, to the other side, we obtain

$$
f_{R}=8\left(1.747 \times 10^{-13}\right) R^{2} /\left(M_{R} c^{2}\right)
$$

Next, because of the Schwarzschild relation, $R=2 G M_{R} / c^{2}$, we can relate the radius of the black hole to its mass, and vice versa. This allows us to rewrite Equation, (3-14), entirely in terms of the mass of the black hole, or entirely, in terms of its radius. In terms of its mass, we find,

$$
f_{R}=\left(3.416 \times 10^{-83}\right) M_{R}
$$

In terms, of its radius, we obtain,

$$
f_{R}=\left(2.303 \times 10^{-56}\right) R
$$

Either way, for a given size, or mass, we now have an expression for, $f_{R}$, the probability density function, just inside the 4-D event horizon.

Once we know, $f_{R}$, we can determine, $\sigma$, the shape parameter. Using Equation, (3-1), it is clear that

$$
f_{R}=\sqrt{(2 / \pi)} 1 / \sigma \exp \left(-R^{2} / 2 \sigma^{2}\right) / \operatorname{erf}(R / \sqrt{2} \sigma)
$$

Unfortunately, the right hand side of this equation is a complicated function of $\sigma$, as $\sigma$ occurs in three separate places. Thus, an analytical solution does not seem possible. We therefore resort to a numerical solution, using a trial and error approach. We step through various $\sigma$ values and look for a match between the right hand side of Equation, (3-17), and either one of Equations, (3-15), or (3-16). This is best achieved by using a spreadsheet, and we will give specific solutions for various black holes.

We will consider three black holes. The first will have the mass which equals that of the sun (not realistic), which we call black hole, $A$, abbreviated, $B H_{A}$. The second will have a mass ten times that of the sun. This we refer to as black hole, $B$, or $B H_{B}$. The third black hole is supermassive black hole with a mass, a million times that of the sun. These kinds of black holes are found at galactic 
centers. This is black hole, $C$, or $B H_{C}$, for short. Using these masses, and the Schwarzschild relation, we find

$$
\begin{aligned}
&\left(M_{A}, M_{B}, M_{C}\right)=\left(1.989 \times 10^{30}, 1.989 \times 10^{31}, 1.989 \times 10^{36}\right) \mathrm{kg} \\
& \rightarrow\left(R_{A}, R_{B}, R_{C}\right)=\left(2.954 \times 10^{3}, 2.954 \times 10^{4}, 2.954 \times 10^{9}\right) \text { meters }
\end{aligned}
$$

We next determine the probability distribution function at radius, $R$, which is, $f_{R}$, for all three black holes using either Equation, (3-15), or Equation, (3-16), whichever is convenient. We find

$$
\left(f_{R, A}, f_{R, B}, f_{R, C}\right)=\left(6.8292 \times 10^{-53}, 6.8292 \times 10^{-52}, 6.8292 \times 10^{-47}\right) \text { meters }^{-1}
$$

Using these values, we next determine the associated $\sigma$ values. We use Equation, (3-17), and our trial and error approach utilizing a spread sheet. We obtain a match for the following shape parameter values, which apply for each of the black holes considered.

$$
\left(\sigma_{A}, \sigma_{B}, \sigma_{C}\right)=\left(1.951 \times 10^{2}, 1.992 \times 10^{3}, 2.241 \times 10^{8}\right) \text { meters }
$$

In this way, we can fix the parameters associated with our choice of probability distribution function. Fixing the $\mu$ values is easy, if we assume a maximum in $f_{r}$ at the origin. If the mode is at, $r=0$, then necessarily, we must set, $\left(\mu_{A}, \mu_{B}, \mu_{C}\right)=(0,0,0)$. Finding the shape parameter, $\sigma$, however, is much more complicated. There does not appear to be an easier way of determining the $\sigma$ value, other than this trial and error approach, and looking for a match in solution.

Once more, we have focused on an isolated, static black hole (ISBH) because our goal in this paper is to illustrate a method, and not work out all possibilities. If we have net radiative inflow, then necessarily, $\mathrm{d} Q / \mathrm{d} t>0$. For this situation, we would have an evolving inside surface temperature at the event horizon, one that is continuously changing with increasing event horizon radius, $R=R(t)$. The inside surface temperature, $T_{1}$, decreases as $R$ increases. The net radiative heat inflow can be determined from the outside blackbody temperature, and the size (or mass) of the black hole. This changing inside surface temperature, on the 4-D side, would translate into other values for, $f_{R}=f_{R}(t)$, and, consequently, $\sigma=\sigma(t)$, which hypothetically can be worked out. We would obtain a different distribution of radiative mass density, or better said, a changing distribution, within the black hole because of Equation, (3-8), and the different $\sigma$, and, $f_{R}$ values. The point is that we would know how to proceed for this more involved situation. In addition, in the interests of keeping the discussion manageable, we have only considered three massive black holes. A whole catalog can be created for differing masses, and different heat inflows.

We close this section by giving the dimensionless ratio of $\sigma$ value, to radius, $R$, for the three black holes under consideration. This is sometimes referred to as the Pearson ratio. It occurs very often, such as in Equations, (3-1), (3-2), (3-3), and (3-4). We divide the shape parameters indicated in Equations, (3-20), by the 
corresponding radii in relations, (3-18). The results are,

$$
\left(\sigma_{A} / R_{A}, \sigma_{B} / R_{B}, \sigma_{C} / R_{C}\right)=(0.0660,0.0674,0.0759)
$$

Even though $\sigma$ is not the standard deviation for a truncated Normal distribution (see Equation, (3-4)), the Pearson ratio is a measure of dispersion. Due to the relatively small values indicated by Equations, (3-21), we can expect a high concentration of radiative mass, radiative pressure, radiative heat density, etc. located close to the center of the black hole. Towards the surface these values will drop down to almost insignificant amounts. This will be made apparent when we construct tables, in Sections 4 and 5, for the three black holes under consideration. There, we will see very clearly how the values are concentrated within a relatively narrow radius, close to the very core of the black hole. It is interesting to notice that this dispersion does not increase significantly, even for a very massive black hole.

\section{Determining Temperature, Radiative Pressure, Internal Energy Densities, and Radiative Forces within the Black Hole}

We have spent some time determining the shape parameter associated with our choice of probability distribution. This will enable us to determine the temperature as a function of radius, within the black hole, itself. Once we have the temperature distribution, we can also find other key thermodynamic quantities of interest within the black hole, and specify these as a function of 4-D radius. We are thinking of the radiative pressure, the internal energy density, the radiative heat density, and the total (radiative mass) energy density. These quantities depend on the temperature and temperature only. Moreover, if temperature is a function of radius, so too, are these quantities.

The radiative force within the black hole will also depend on temperature, but, in addition, on the surface area. The surface area, in turn, depends on the 4-D radius. By definition, according to Equation, (2-23), $F_{r} \equiv p_{r} A_{r}=p_{r} 2 \pi^{2} r^{3}$, where we have dropped the superscript on, $F_{r}, p_{r}, A_{r}$, for ease of writing. Unless otherwise indicated, all quantities in this section, and the next, are 4-dimensional quantities. Therefore, we will dispense with the superscripts, unless we have to distinguish between 3-D and 4-D quantities. At radius, $r$, the force, $F_{r}$, is a radiative force pushing the surface area, $A_{r}=2 \pi^{2} r^{3}$, out. At radius, $r+\mathrm{d} r$, we have another radiative force, $F_{r+\mathrm{d} r}$, which pushes the surface area, $A_{r+\mathrm{d} r}$, at $r+\mathrm{d} r$, from the outside in, because it acts from the top down. Together with gravity, which also pulls the layer between radii, $r$, and, $r+\mathrm{d} r$, in, these forces guarantee for hydrostatic equilibrium, according to Equation, (2-26). Layer by layer, the black hole is thus kept in stable equilibrium. For a given radius, $r$, the forces, $F_{r}$, and $F_{r+\mathrm{d} r}$, depend only on temperature, $T_{r}=T(r)$. And because, $T_{r}=T(r)>T_{r+\mathrm{d} r}=T(r+\mathrm{d} r)$, we have a greater radiative force pushing out at radius, $r$, than pushing in at radius, $r+\mathrm{d} r$. In other words, $F_{r}>F_{r+\mathrm{d} r}$. Refer to Equation, (2-26). 
From Section 2, we know that Equation, (2-17), holds. With this equation we can calculate the internal energy density at any temperature, $T_{r}$. Once we have the internal energy density, it is easy to calculate the radiative pressure, the heat density, and the entropy density. We use Equations, (2-18). To find the total energy "density", and the radiative mass density, we employ Equations, (2-20), and, (2-21). Thus, the temperature, $T_{r}$, is needed for all these quantities. But, $T_{r}$, can be determined. We start with Equation, (2-25). This equation can be rewritten in the forms,

$$
\begin{gathered}
\rho_{r} A_{r}=f_{r} M_{R} \\
\left(e_{r} / c^{2}\right) A_{r}=f_{r} M_{R} \\
(10 / 4)\left(u_{r} / c^{2}\right) A_{r}=f_{r} M_{R} \\
(10 / 4)\left(a^{(4)} T_{r}^{5}\right) / c^{2} A_{r}=f_{r} M_{R} \\
(10 / 4)\left(a^{(4)} T_{r}^{5}\right) / c^{2} 2 \pi^{2} r^{3}=\sqrt{(2 / \pi)} 1 / \sigma \exp \left(-r^{2} /\left(2 \sigma^{2}\right)\right) 1 / \operatorname{erf}(R /(\sqrt{2} \sigma)) M_{R}
\end{gathered}
$$

For the $2^{\text {nd }}$ line, we substituted the first line in Equation, $(2-21)$. In the $3^{\text {rd }}$ line, we employed the last equality in relations, (2-22). The $1^{\text {st }}$ line in Equation, (2-17), was invoked for the $4^{\text {th }}$ line in Equations, (4-1). And finally for the $5^{\text {th }}$ line, we substituted, Equation, (3-1), on the right hand side, and our, by now familiar, expression for the 4-dimensional surface area, $A_{r}=2 \pi^{2} r^{3}$, on the left hand side. Upon inspection of the right hand side in the last of the series of Equations, in, (4-1), we see that this depends on the variables, $r, R$, and, $\sigma$. The left hand side depends on the temperature, $T_{r}$, and the radius, $r$. Therefore, for a given $r$ value, we can use this equation to determine the temperature as a function of radius. All that we need is the shape parameter and the mass, or size, of the black hole. Those values were determined in the previous section for the various specific black holes under consideration.

Equation, (4-1), however, is a rather complicated function. Another way to calculate the temperature, $T_{r}$, within the black hole is less complicated, and offers additional insight. We shall follow this approach, even though it is less direct, as it will allow us to derive the other quantities in a more systematic manner. We proceed in a series of steps, and use the three black holes introduced in the previous section, to illustrate the results. In this way numerical comparisons can be made, and highlighted. The three massive black holes under consideration, are those specified in Equations, (3-18), where we have specified both their masses, and their associated radii.

The first step is to determine the temperature just inside the event horizon, $T_{1}$. For this we use our generalized Stefan-Boltzmann law, derived in reference [5]. The equation reads,

$$
(1 / c) \mathrm{d} Q / \mathrm{d} t=(4 / 3) a^{(3)} T_{2}^{4} 4 \pi R^{2}-(5 / 4) a^{(4)} T_{1}^{5} 2 \pi^{2} R^{3}
$$

In this equation, the constants, $a^{(3)}$, and $a^{(4)}$, have the numerical values in MKS units, 


$$
\begin{gathered}
a^{(4)} \equiv(3 \pi / 2 c) \sigma^{(4)}=4.7481 \times 10^{-13} \\
a^{(3)} \equiv(4 / c) \sigma^{(3)}=7.5657 \times 10^{-16}
\end{gathered}
$$

The quantity, $\sigma^{(3)}=5.67 \times 10^{-8}$ Watts $/ \mathrm{m}^{2} \cdot \mathrm{K}^{4}$, is the Stefan-Boltzmann constant in three dimensional space. In 4-D space, its extension [8] is given by, $\sigma^{(4)}=3.021 \times 10^{-5}$ Watts $/ \mathrm{m}^{3} \cdot \mathrm{K}^{5}$. In Equation, (4-2), the quantity, $\mathrm{d} Q / \mathrm{d} t$, is the net radiative heat entering the black hole from the 3 -D side. We have shown in a previous work [5] that, $\mathrm{d} Q / \mathrm{d} t \geq 0$, is a direct consequence of our 4-D black hole radiation model, as we are ignoring/discounting radiative evaporation, for example, due to Hawking radiation. These are second order effects, at best, if they exist, as shown in reference [5]. The temperatures, $T_{2}$, and $T_{1}$, in Equation, (4-2), are the temperatures just outside, and just inside, the event horizon, respectively. The temperature, $T_{2}$, is on the 3 -dimensional side, whereas, the temperature, $T_{1}$, is on the 4-D side within the black hole. The, $R$, is, of course, the radius of the black hole event horizon, proportional to its mass, because of the Schwarzschild relation.

We notice that Equation, (4-2), contains two terms on the right hand side. The first term on the right hand side, is the radiative heat leaving 3-D space, and entering the 4-D black hole. We assume that the event horizon is infinitely thin. The second term on the right hand side is the radiative heat exiting the 4-D black hole, and entering 3-D space. Both propagate at velocity, $c$. Another way of writing Equation, (4-2), is thus

$$
1 / c \mathrm{~d} Q / \mathrm{d} t=1 / c \mathrm{~d} Q^{(3)} / \mathrm{d} t-1 / c \mathrm{~d} Q^{(4)} / \mathrm{d} t
$$

In this expression, $\mathrm{d} Q^{(3)} / \mathrm{d} t$ is the radiative heat streaming into the black hole, and, $\mathrm{d} Q^{(4)} / \mathrm{d} t$, is the radiative heat flowing out. This expression leads to a necessarily positive radiative surface tension, and was a primary motivation for the construction of a black hole as a 4-D object.

There are other ways to write Equation, (4-2), the generalized Stefan-Boltzmann law. A particularly straight-forward way is in terms of radiative pressure, and radiative force. In this formulation,

$$
(1 / c) \mathrm{d} Q / \mathrm{d} t=4 p_{2}^{(3)} A_{R}^{(3)}-5 p_{1}^{(4)} A_{R}^{(4)}
$$

here, the surface areas, $A_{R}^{(3)}=4 \pi R^{2}$, and, $A_{R}^{(4)}=2 \pi^{2} R^{3}$, are the 3-dimensional and 4-dimensional surface areas, respectively, at the event horizon. Also, it is to be noticed that the 3 -D radiative pressure, $p_{2}^{(3)}=1 / 3 u_{2}^{(3)}=1 / 3 a^{(3)} T_{2}^{4}$. Thus, $p_{2}^{(3)}$, is defined in terms of the temperature right outside the event horizon. By contrast, the 4-D radiative pressure is defined in terms of a different temperature, $T_{1}$. There, we have, $p_{1}^{(4)}=1 / 4 u_{1}^{(4)}=1 / 4 a^{(4)} T_{1}^{5}$, where, $T_{1}$, is the temperature right inside the event horizon, within the 4 -D black hole.

Equation, (4-6), for net radiative heat inflow, can also be rewritten as,

$$
(1 / c) \mathrm{d} Q / \mathrm{d} t=4 F_{R}^{(3)}-5 F_{R}^{(4)}
$$

where, 


$$
F_{R}^{(3)} \equiv p_{2}^{(3)} A_{R}^{(3)} \text { and } F_{R}^{(4)} \equiv p_{1}^{(4)} A_{R}^{(4)}
$$

The particular factors of, 4, and, 5, in Equations, (4-6), and, (4-7), are imposed upon us by the dimensionality of the differing spaces. The radiative force, $F_{R}^{(3)}$, acts on the event horizon and pulls the event horizon membrane radially in. The radiative force, $F_{R}^{(4)}$, by contrast, acts on the infinitely thin event horizon, and pushes the membrane radially out. Because $\mathrm{d} Q / \mathrm{d} t \geq 0$, we see that,

$4 F_{R}^{(3)} \geq 5 F_{R}^{(4)}$. The radiative surface tension, defined as, $F_{\mathrm{ST} \text { radiative }} \equiv F_{R}^{(3)}-F_{R}^{(4)}$, was thus shown to be always positive.

We will next assume that there is no net inflow. In other words, $\mathrm{d} Q / \mathrm{d} t=0$, and we have what we refer to as an isolated, static black hole (ISBH). Net inflow will be treated in another paper and it will be easy to extend these ideas once we understand the process for an isolated static black hole. For an ISBH, we assume that the outside temperature is, $T_{2}=2.725 \mathrm{~K}$, a working hypothesis. This cannot be proven, just surmised, because, at this small temperature, net inflows will not lead to appreciable expansion. In other words, expansion, if it existed, cannot be observed as it would be so slight. As such, at $T_{2}=2.725 \mathrm{~K}$, we may, or, may not, have inflow. We assume no inflow. Under this assumption, the CMB photons surrounding the black hole will lead to a temperature, called the equilibrium temperature, just inside the event horizon equal to,

$$
T_{1}^{5} R=0.0597 \text { or } T_{1}=0.569 R^{-1 / 5}
$$

(ISBH) $(4-9 a, b)$

This is obtained by solving Equation, (4-2), after we set the left hand side equal to zero. Remember that, for, $T_{2}=2.725 \mathrm{~K}$, we assume we have an isolated, static black hole.

Even though Equations, (4-9a,b), were derived for an isolated, static black hole, they tell us how to proceed for a dynamic black hole (DBH), with $\mathrm{d} Q / \mathrm{d} t>0$. For a different outside surface temperature, $T_{2}>2.725 \mathrm{~K}$, we will have net inflow, and, $R=R(t)$. The relation to be used here for $T_{1}$ is the same as before, except that now, $R$ is evolving, and getting larger. As a consequence, $T_{1}$, will start to decrease by the equations above. The radius will start to increase with net heat inflow, but until it has achieved a new value, we must assume that the inside temperature hasn't caught up yet. In other words it is still the temperature as determined by Equation (4-9b). We can argue as follows. The inside surface temperature determines, through the shape parameter $\sigma$, the inner mass distribution within the black hole. This, in turn, determines its total mass. The total mass determines the total radius. And the total radius relates back to the inside temperature by use of Equations, (4-9a,b). Until such time that the radius increases, $T_{1}$, will be fixed by the above equation. In a sense, a dynamic black hole is an evolving set of isolated, static black holes, each with a different radius. The energy absorbed will determine the final radius, irrespective of how much time it takes. The rate of inflow will determine that.

If we go back in cosmological time, the CMB temperature was higher. Thus, Equations, (4-9a,b), would have to be modified as we assumed that the CMB temperature was $2.725 \mathrm{~K}$ to obtain the factors of 0.0597 and 0.569 in these ex- 
pressions. For higher (or lower) CMB temperatures, we have to scale both factors as follows:

$$
\begin{gathered}
(0.0597) \rightarrow\left(T_{C M B} / 2.725\right)^{4}(0.0597), \text { and, } \\
(0.569) \rightarrow\left(T_{C M B} / 2.725\right)^{4 / 5}(0.569),
\end{gathered}
$$

to take into account different $\mathrm{CMB}$ temperatures. If we go back in cosmological time, for the same radius, the equilibrium inside surface temperature was higher. That means for the same outside temperature load, $T_{2}>T_{C M B}$, inflow must have been less. In future epoch, it will be greater under the same conditions. As, $T_{C M B} \rightarrow 0$, we obtain (reproduce) the permanent inflow model discussed in the introduction.

From Equations, (4-9a), we see that the mass (size) of a black hole determines the inside surface temperature. The greater the radius, the less of an inside surface temperature, we will have. For the three black holes considered in relations, (3-18), upon using Equations, (4-9a), we find that

$$
\left(T_{1, A}, T_{1, B}, T_{1, C}\right)=(0.115 \mathrm{~K}, 0.0726 \mathrm{~K}, 0.00726 \mathrm{~K})
$$

These temperatures are many orders of magnitude greater than the corresponding Hawking temperatures, and yet, they are below the minimum outside surface temperature of, $2.725 \mathrm{~K}$. For example, the predicted Hawking temperature for a black hole having a mass equal to that of the sun is only, $62 \mathrm{nK}$, which is much less than $0.115 \mathrm{~K}$, shown in Equation (4-10). But then, the space considered was 3-dimensional, and a totally different specific process was considered. We also remark that the variation in temperature between the various massive black holes is not as dramatic as might be expected. This is due to the $R^{-1 / 5}$ factor in Equation, (4-9b), which in effect, is equivalent to $M_{R}^{-1 / 5}$ because of the Schwarzschild proportionality between mass and radius. The Hawking temperature, by contrast, equals, $T_{H}=\hbar c^{3} /\left(8 \pi G M k_{B}\right)$, and therefore is proportional to $M_{R}^{-1}$. His temperature, therefore, falls off much more dramatically than ours, as one increases black hole mass. This first step of finding the inside 4-D surface temperature, really consists of applying Equation, (4-9a), or, Equation, (4-9b), to find the inside surface temperature.

The second step consists of strict calculations. In this step, we determine the internal energy density, the radiative pressure, the radiative heat density, and the total entropy density, defined by Equations, (2-17), and, (2-18). These quantities are all to be calculated just inside the event horizon, where we know the temperature, $T_{1}$. For the internal energy density, $u_{R}=u_{1}=u_{1}^{(4)}$, we use Equation, (2-17). The respective temperatures are listed in Equations, (4-10). The results for the three black holes considered are thus,

$$
\left(u_{1, A}, u_{1, B}, u_{1, C}\right)=\left(9.60 \times 10^{-18}, 9.60 \times 10^{-19}, 9.60 \times 10^{-24}\right) \mathrm{J} / \mathrm{m}^{4}
$$

These values are very small, but then the temperature, just within the event horizon is very small. We can now use Equations, (2-18), to find the radiative pressures, the heat densities, the entropy densities. The results, with appropriate 
units, are:

$$
\begin{gathered}
\left(p_{1, A}, p_{1, B}, p_{1, C}\right)=\left(2.40 \times 10^{-18}, 2.40 \times 10^{-19}, 2.40 \times 10^{-24}\right) \mathrm{N} / \mathrm{m}^{3} \\
\left(q_{1, A}, q_{1, B}, q_{1, C}\right)=\left(1.20 \times 10^{-17}, 1.20 \times 10^{-18}, 1.20 \times 10^{-23}\right) \mathrm{J} / \mathrm{m}^{4} \\
\left(s_{1, A}, s_{1, B}, s_{1, C}\right)=\left(1.04 \times 10^{-16}, 1.65 \times 10^{-17}, 1.65 \times 10^{-21}\right) \mathrm{J} /\left(\mathrm{m}^{4} \cdot \mathrm{K}\right)
\end{gathered}
$$

Equations, (4-11), (4-12), (4-13), and (4-14), hold for an isolated, static black hole (ISBH), and until the radius has changed, for a dynamic black hole (DBH), as well.

In step three, we calculate the total (radiative mass) energy "density", the 4-D mass density, and the radiative force, all at, $r=R$, just inside the 4-D event horizon. For the total energy density, we use Equation, (2-20), in conjunction with Equations, (4-11), (4-12), and, (4-13). The results are:

$$
\left(e_{1, A}, e_{1, B}, e_{1, C}\right)=\left(2.40 \times 10^{-17}, 2.40 \times 10^{-18}, 2.40 \times 10^{-23}\right)
$$

These values, numerically, are twice the radiative heat density, which confirms the second equality in the Equations, (2-22). In fact, all the equalities in Equation, $(2-22)$, are found to hold true. For the mass density, we can use the first line in Equation, (2-21), together with Equation, (4-15). Here, we obtain

$$
\left(\rho_{1, A}, \rho_{1, B}, \rho_{1, C}\right)=\left(2.67 \times 10^{-34}, 2.67 \times 10^{-35}, 2.67 \times 10^{-40}\right) \mathrm{kg} / \mathrm{m}^{4}
$$

These values are exceedingly low, but then we are in $4-\mathrm{D}$ space. And, for the radiative force, Equation, (2-23), can be utilized where we use the radiative pressure values indicated in Equations, (4-12), together with the radii specified in Equations, (2-18). The radii are needed to calculate the 4 -D surface areas using the formula, $A_{R}=2 \pi^{2} R^{3}$. The net results of these calculations give,

$$
\left(F_{1, A}, F_{1, B}, F_{1, C}\right)=\left(1.22 \times 10^{-6}, 1.22 \times 10^{-4}, 1.22 \times 10^{6}\right) \text { Newtons }
$$

These 4-D radiative forces, pushing out against the event horizon, seem incredibly small, considering the fact that we are dealing with very massive objects. They, in fact, are. We should keep in mind, however, that they push against a greater 4-D surface area. Radiative forces, as it will turn out, are insignificant when compared to gravitational forces, except in the most extreme of circumstances, close to the event horizon of a black hole. This will be seen shortly. Within the interior however, radiative forces will have to be comparable to gravitational forces for hydrostatic equilibrium. See Equation, (2-26). On the surface we do not have hydrostatic equilibrium; we have, instead, an intrinsic surface tension which is non-zero due to the changing dimensionality of space. Equations, (4-15), (4-16), and (4-17), hold only at the event horizon, just inside the 4-D black hole, because it is there that we have established a temperature. As we shall see shortly, all the values indicated by Equations, (4-11), through to, (4-17), will increase dramatically as we enter the interior of the black hole. The subscript, " 1 " in all these relations can be interchanged with, " $R$ ", because the temperature, $T_{1}$, holds at radius $R$, inside the black hole. As examples, we can write 
$p_{1, A}=p_{R, A}, p_{1, B}=p_{R, B}, q_{1, C}=q_{R, C}$, etc.

Thus far, all the pressures, densities, and forces are given at radius, $R$, because at that radius, we know the temperature. We now look into the interior, where $r<R$. In step 4 , we will calculate the mass density as a function of radius, $\rho_{r}=\rho(r)$. For that, we will employ Equation, (3-8). This can be written in the form,

$$
\left(\rho_{r} r^{3}\right) /\left(\rho_{R} R^{3}\right)=f_{r} / f_{R}=\exp \left[\left(R^{2}-r^{2}\right) / 2 \sigma^{2}\right]
$$

We notice that in the limit where, $r \rightarrow 0$, the right hand side is non-zero. Therefore the left hand side must also be finite in this limit. This can only mean one thing for the left hand side, namely, that $\rho_{r}$ increases more rapidly, than $r^{3}$ decreases, in this limit. Also remember that $\rho_{r}$ is really a density. A density, a-priori, requires a finite volume, however small. The pdf chosen is seen to give us a finite result for the right hand side given this limit. And so, without further analysis, we must accept that the left hand side is also finite in the limit where, $r \rightarrow 0$.

For each of the black holes considered, we know the shape parameter, $\sigma$, and the total radii, $R$. Therefore, for a specified $r<R$ value, we can evaluate the right hand side of Equation, (4-18). We also know the $\rho_{R}$ values on the left hand side, which hold at the surface. These are given by Equations, (4-16). We can therefore determine the inner radiative mass density, $\rho_{r}$, as a function of radius. These results have to be given in table form, because we are considering separate, $r / R$ values. The tables are presented in Tables 1-3. Table 1 , applies to a black hole having a mass equal to that of the sun. Table 2, holds for a black hole, having a mass ten times that of the sun. And Table 3 refers to the $3^{\text {rd }}$ black hole under consideration, one having a mass a million times that of the sun. All tables will increase the radius in steps indicated. And so, in the first column, we have $r=0.03 R, 0.06 R, 0.09 R, \cdots$. Notice that we do not start with, $r=0$, as it is meaningless to define a density, or for that matter, a temperature, for zero volume. We therefore start with an almost infinitesimally small, but finite radius, $r=10^{-6} R$. The associated, $\rho_{r}$, values are calculated under column 3 in these tables. Column 2 is reserved for, $f_{r} / f_{R}=\exp \left[\left(R^{2}-r^{2}\right) / 2 \sigma^{2}\right]$, which is specific to our choice of pdf.

The fifth step is about calculating the total energy "density", using the first line in relation, (2-21). And then, by using Equations, (2-22), we evaluate the radiative pressure, the radiative heat density, and the internal energy density. Since the 4-D mass density, $\rho_{r}$, within the black hole is a function of radius, $r$, so too are, $e_{r}, p_{r}, q_{r}$ and, $u_{r}$. The total energy density is given by the simple relation, $e_{r}=\rho_{r} c^{2}$, where $c$ is the speed of light. Again, because we are dealing with functions that depend on, $r / R$, we have to give the results in table form. These are presented in Tables 1-3, where each table refers to a black hole of a specific mass. The values for, $u_{r}, p_{r}$ and, $q_{r}$, are highlighted under columns, 4,5 , and 6 , respectively. The values for total (radiative mass) energy densities, $e_{r}$, are not 
Table 1. For a blackhole having 1 solar mass.

\begin{tabular}{|c|c|c|c|c|c|c|c|c|c|c|c|}
\hline$r / R$ & $\mathrm{f}_{\mathrm{r}} / \mathrm{f}_{\mathrm{R}}$ & $\rho_{\mathrm{r}}$ & $\mathbf{u}_{\mathrm{r}}$ & $\mathrm{p}_{\mathrm{r}}$ & $\mathrm{q}_{\mathrm{r}}$ & $\mathrm{T}_{\mathrm{r}}$ & $s_{\mathrm{r}}$ & $\mathbf{F}_{\mathrm{r}}$ & $\mathrm{dT}_{\mathrm{r}} / \mathrm{dr}$ & $\mathrm{C}_{\mathrm{r}}=\mathrm{du}_{\mathrm{r}} / \mathrm{dT}_{\mathrm{r}}$ & $\mathrm{du}_{\mathrm{r}} / \mathrm{dr}$ \\
\hline & & $\left(\mathrm{kg} / \mathrm{m}^{4}\right)$ & (Joules $/ \mathrm{m}^{4}$ ) & $\begin{array}{c}\text { (Newtons/ } \\
\mathrm{m}^{3} \text { ) }\end{array}$ & $\left(\right.$ Joules $\left./ \mathrm{m}^{4}\right)$ & $(\mathrm{K})$ & $\begin{array}{c}\text { (Joules/ } \\
\left.\left(\mathrm{m}^{4} \mathrm{~K}\right)\right)\end{array}$ & (Newtons) & $(\mathrm{K} / \mathrm{m})$ & $\begin{array}{c}\text { (Joules/ } \\
\left.\mathrm{m}^{4} \mathrm{~K}\right)\end{array}$ & $\left(\right.$ Joules $/ \mathrm{m}^{5}$ ) \\
\hline 0.000001 & $5.99 \mathrm{E}+49$ & $1.60 \mathrm{E}+34$ & $5.75 \mathrm{E}+50$ & $1.44 \mathrm{E}+50$ & $7.18 \mathrm{E}+50$ & $4.14 \mathrm{E}+12$ & $1.74 \mathrm{E}+38$ & $7.31 \mathrm{E}+43$ & $-8.40 \mathrm{E}+14$ & $6.95 \mathrm{E}+38$ & $-5.84 \mathrm{E}+53$ \\
\hline 0.03 & $5.40 \mathrm{E}+49$ & $5.34 \mathrm{E}+20$ & $1.92 \mathrm{E}+37$ & $4.80 \mathrm{E}+36$ & $2.40 \mathrm{E}+37$ & $8.34 \mathrm{E}+09$ & $2.88 \mathrm{E}+27$ & $6.59 \mathrm{E}+43$ & $-6.04 \mathrm{E}+07$ & $1.15 \mathrm{E}+28$ & $-6.95 E+35$ \\
\hline 0.06 & $3.96 \mathrm{E}+49$ & $4.90 \mathrm{E}+19$ & $1.76 \mathrm{E}+36$ & $4.40 \mathrm{E}+35$ & $2.20 \mathrm{E}+36$ & $5.17 \mathrm{E}+09$ & $4.25 \mathrm{E}+26$ & $4.84 \mathrm{E}+43$ & $-2.23 \mathrm{E}+07$ & $1.70 \mathrm{E}+27$ & $-3.80 \mathrm{E}+34$ \\
\hline 0.09 & $2.37 \mathrm{E}+49$ & $8.67 \mathrm{E}+18$ & $3.12 \mathrm{E}+35$ & $7.79 \mathrm{E}+34$ & $3.89 \mathrm{E}+35$ & $3.66 \mathrm{E}+09$ & $1.06 \mathrm{E}+26$ & $2.89 \mathrm{E}+43$ & $-1.34 \mathrm{E}+07$ & $4.26 \mathrm{E}+26$ & $-5.69 \mathrm{E}+33$ \\
\hline 0.12 & $1.15 \mathrm{E}+49$ & $1.78 \mathrm{E}+18$ & $6.38 \mathrm{E}+34$ & $1.60 \mathrm{E}+34$ & $7.98 \mathrm{E}+34$ & $2.67 \mathrm{E}+09$ & $2.99 \mathrm{E}+25$ & $1.40 \mathrm{E}+43$ & $-9.47 \mathrm{E}+06$ & $1.20 \mathrm{E}+26$ & $-1.13 \mathrm{E}+33$ \\
\hline 0.15 & $4.54 \mathrm{E}+48$ & $3.59 \mathrm{E}+17$ & $1.29 \mathrm{E}+34$ & $3.23 \mathrm{E}+33$ & $1.61 \mathrm{E}+34$ & $1.94 \mathrm{E}+09$ & $8.34 \mathrm{E}+24$ & $5.55 \mathrm{E}+42$ & $-7.13 \mathrm{E}+06$ & $3.34 \mathrm{E}+25$ & $-2.38 \mathrm{E}+32$ \\
\hline 0.18 & $1.46 \mathrm{E}+48$ & $6.69 \mathrm{E}+16$ & $2.40 \mathrm{E}+33$ & $6.01 \mathrm{E}+32$ & $3.00 \mathrm{E}+33$ & $1.38 \mathrm{E}+09$ & $2.17 \mathrm{E}+24$ & $1.78 \mathrm{E}+42$ & $-5.42 \mathrm{E}+06$ & $8.69 \mathrm{E}+24$ & $-4.71 \mathrm{E}+31$ \\
\hline 0.21 & $3.82 \mathrm{E}+47$ & $1.10 \mathrm{E}+16$ & $3.96 \mathrm{E}+32$ & $9.90 \mathrm{E}+31$ & $4.95 E+32$ & $9.64 \mathrm{E}+08$ & $5.13 \mathrm{E}+23$ & $4.66 \mathrm{E}+41$ & $-4.08 \mathrm{E}+06$ & $2.05 \mathrm{E}+24$ & $-8.37 \mathrm{E}+30$ \\
\hline 0.24 & $8.13 \mathrm{E}+46$ & $1.57 \mathrm{E}+15$ & $5.64 \mathrm{E}+31$ & $1.41 \mathrm{E}+31$ & $7.06 \mathrm{E}+31$ & $6.53 \mathrm{E}+08$ & $1.08 \mathrm{E}+23$ & $9.93 \mathrm{E}+40$ & $-2.99 \mathrm{E}+06$ & $4.32 \mathrm{E}+23$ & $-1.29 \mathrm{E}+30$ \\
\hline 0.27 & $1.41 \mathrm{E}+46$ & $1.91 \mathrm{E}+14$ & $6.86 \mathrm{E}+30$ & $1.72 \mathrm{E}+30$ & $8.58 \mathrm{E}+30$ & $4.29 \mathrm{E}+08$ & $2.00 \mathrm{E}+22$ & $1.72 \mathrm{E}+40$ & $-2.12 \mathrm{E}+06$ & $8.01 \mathrm{E}+22$ & $-1.70 \mathrm{E}+29$ \\
\hline 0.3 & $1.98 \mathrm{E}+45$ & $1.96 \mathrm{E}+13$ & $7.05 \mathrm{E}+29$ & $1.76 \mathrm{E}+29$ & $8.81 \mathrm{E}+29$ & $2.72 \mathrm{E}+08$ & $3.24 \mathrm{E}+21$ & $2.42 \mathrm{E}+39$ & $-1.45 \mathrm{E}+06$ & $1.30 \mathrm{E}+22$ & $-1.88 \mathrm{E}+28$ \\
\hline 0.5 & $2.15 \mathrm{E}+37$ & $4.60 \mathrm{E}+04$ & $1.65 \mathrm{E}+21$ & $4.13 \mathrm{E}+20$ & $2.07 \mathrm{E}+21$ & $5.11 \mathrm{E}+06$ & $4.04 \mathrm{E}+14$ & $2.63 \mathrm{E}+31$ & $-4.17 \mathrm{E}+04$ & $1.62 \mathrm{E}+15$ & $-6.75 E+19$ \\
\hline 1 & $1.00 \mathrm{E}+00$ & $2.67 \mathrm{E}-34$ & $9.60 \mathrm{E}-18$ & $2.40 \mathrm{E}-18$ & $1.20 \mathrm{E}-17$ & $1.15 \mathrm{E}-01$ & $1.04 \mathrm{E}-16$ & $1.22 \mathrm{E}-06$ & $-1.81 \mathrm{E}-03$ & $4.17 \mathrm{E}-16$ & $-7.54 \mathrm{E}-19$ \\
\hline
\end{tabular}

Table 2. For a Blackhole having 10 solar masses.

\begin{tabular}{|c|c|c|c|c|c|c|c|c|c|c|c|}
\hline $\mathrm{r} / \mathrm{R}$ & $\mathrm{f}_{\mathrm{r}} / \mathrm{f}_{\mathrm{R}}$ & $\rho_{\mathrm{r}}$ & $\mathbf{u}_{\mathrm{r}}$ & $\mathrm{p}_{\mathrm{r}}$ & $\mathrm{q}_{\mathrm{r}}$ & $\mathrm{T}_{\mathrm{r}}$ & $\mathbf{s}_{\mathrm{r}}$ & $\mathbf{F}_{\mathrm{r}}$ & $\mathbf{d T}_{\mathrm{r}} / \mathrm{dr}$ & $\mathrm{C}_{\mathrm{r}}=\mathrm{du}_{\mathrm{r}} / \mathrm{dT}_{\mathrm{r}}$ & $d \mathbf{u}_{\mathrm{r}} / \mathrm{dr}$ \\
\hline & & $\left(\mathrm{kg} / \mathrm{m}^{4}\right)$ & $($ Joules/m²) & $\begin{array}{c}\text { (Newtons/ } \\
\mathrm{m}^{3} \text { ) }\end{array}$ & (Joules/m $)$ & $(\mathrm{K})$ & $\begin{array}{l}\text { (Joules/ } \\
\left.\left(\mathrm{m}^{4} \mathrm{~K}\right)\right)\end{array}$ & (Newtons) & $(\mathrm{K} / \mathrm{m})$ & $\begin{array}{c}\text { (Joules/ } \\
\left.\mathrm{m}^{4} \mathrm{~K}\right)\end{array}$ & (Joules $/ \mathrm{m}^{5}$ ) \\
\hline 0.000001 & $5.87 \mathrm{E}+47$ & $1.57 \mathrm{E}+31$ & $5.63 \mathrm{E}+47$ & $1.41 \mathrm{E}+47$ & $7.04 \mathrm{E}+47$ & $1.03 \mathrm{E}+12$ & $6.80 \mathrm{E}+35$ & $7.16 \mathrm{E}+43$ & $-2.10 \mathrm{E}+13$ & $2.72 \mathrm{E}+36$ & $-5.72 \mathrm{E}+49$ \\
\hline 0.03 & $5.31 \mathrm{E}+47$ & $5.25 \mathrm{E}+17$ & $1.89 \mathrm{E}+34$ & $4.72 \mathrm{E}+33$ & $2.36 \mathrm{E}+34$ & $2.09 \mathrm{E}+09$ & $1.13 E+25$ & $6.49 \mathrm{E}+43$ & $-1.51 \mathrm{E}+06$ & $4.52 \mathrm{E}+25$ & $-6.81 \mathrm{E}+31$ \\
\hline 0.06 & $3.95 \mathrm{E}+47$ & $4.88 \mathrm{E}+16$ & $1.75 \mathrm{E}+33$ & $4.39 \mathrm{E}+32$ & $2.19 \mathrm{E}+33$ & $1.30 \mathrm{E}+09$ & $1.69 \mathrm{E}+24$ & $4.82 \mathrm{E}+43$ & $-5.56 \mathrm{E}+05$ & $6.75 \mathrm{E}+24$ & $-3.75 E+30$ \\
\hline 0.09 & $2.41 \mathrm{E}+47$ & $8.81 \mathrm{E}+15$ & $3.17 \mathrm{E}+32$ & $7.92 \mathrm{E}+31$ & $3.96 \mathrm{E}+32$ & $9.22 \mathrm{E}+08$ & $4.29 \mathrm{E}+23$ & $2.94 \mathrm{E}+43$ & $-3.32 \mathrm{E}+05$ & $1.72 \mathrm{E}+24$ & $-5.70 \mathrm{E}+29$ \\
\hline 0.12 & $1.20 \mathrm{E}+47$ & $1.86 \mathrm{E}+15$ & $6.68 \mathrm{E}+31$ & $1.67 \mathrm{E}+31$ & $8.36 \mathrm{E}+31$ & $6.76 \mathrm{E}+08$ & $1.24 \mathrm{E}+23$ & $1.47 \mathrm{E}+43$ & $-2.35 \mathrm{E}+05$ & $4.95 \mathrm{E}+23$ & $-1.16 \mathrm{E}+29$ \\
\hline 0.15 & $4.94 \mathrm{E}+46$ & $3.91 \mathrm{E}+14$ & $1.40 \mathrm{E}+31$ & $3.51 \mathrm{E}+30$ & $1.76 \mathrm{E}+31$ & $4.95 \mathrm{E}+08$ & $3.55 \mathrm{E}+22$ & $6.03 E+42$ & $-1.77 \mathrm{E}+05$ & $1.42 \mathrm{E}+23$ & $-2.52 \mathrm{E}+28$ \\
\hline 0.18 & $1.66 \mathrm{E}+46$ & $7.61 \mathrm{E}+13$ & $2.74 \mathrm{E}+30$ & $6.84 \mathrm{E}+29$ & $3.42 \mathrm{E}+30$ & $3.57 \mathrm{E}+08$ & $9.59 \mathrm{E}+21$ & $2.03 \mathrm{E}+42$ & $-1.36 \mathrm{E}+05$ & $3.84 \mathrm{E}+22$ & $-5.21 \mathrm{E}+27$ \\
\hline 0.21 & $4.59 \mathrm{E}+45$ & $1.32 \mathrm{E}+13$ & $4.76 \mathrm{E}+29$ & $1.19 \mathrm{E}+29$ & $5.94 \mathrm{E}+29$ & $2.51 \mathrm{E}+08$ & $2.37 \mathrm{E}+21$ & $5.60 \mathrm{E}+41$ & $-1.03 \mathrm{E}+05$ & $9.46 \mathrm{E}+21$ & $-9.74 \mathrm{E}+26$ \\
\hline 0.24 & $1.04 \mathrm{E}+45$ & $2.01 \mathrm{E}+12$ & $7.22 \mathrm{E}+28$ & $1.80 \mathrm{E}+28$ & $9.02 \mathrm{E}+28$ & $1.72 \mathrm{E}+08$ & $5.24 \mathrm{E}+20$ & $1.27 \mathrm{E}+41$ & $-7.62 \mathrm{E}+04$ & $2.09 \mathrm{E}+21$ & $-1.60 \mathrm{E}+26$ \\
\hline 0.27 & $1.93 \mathrm{E}+44$ & $2.62 \mathrm{E}+11$ & $9.42 \mathrm{E}+27$ & $2.36 \mathrm{E}+27$ & $1.18 \mathrm{E}+28$ & $1.15 \mathrm{E}+08$ & $1.03 \mathrm{E}+20$ & $2.36 \mathrm{E}+40$ & $-5.47 \mathrm{E}+04$ & $4.11 \mathrm{E}+20$ & $-2.25 \mathrm{E}+25$ \\
\hline 0.3 & $2.95 \mathrm{E}+43$ & $2.91 \mathrm{E}+10$ & $1.05 \mathrm{E}+27$ & $2.62 \mathrm{E}+26$ & $1.31 \mathrm{E}+27$ & $7.39 \mathrm{E}+07$ & $1.77 \mathrm{E}+19$ & $3.60 \mathrm{E}+39$ & $-3.80 \mathrm{E}+04$ & $7.08 \mathrm{E}+19$ & $-2.69 \mathrm{E}+24$ \\
\hline 0.5 & $6.70 \mathrm{E}+35$ & $1.43 \mathrm{E}+02$ & $5.15 \mathrm{E}+18$ & $1.29 \mathrm{E}+18$ & $6.43 \mathrm{E}+18$ & $1.61 \mathrm{E}+06$ & $3.99 \mathrm{E}+12$ & $8.18 \mathrm{E}+31$ & $-1.26 \mathrm{E}+03$ & $1.60 \mathrm{E}+13$ & $-2.02 \mathrm{E}+16$ \\
\hline 1 & $1.00 \mathrm{E}+00$ & $2.67 \mathrm{E}-35$ & $9.60 \mathrm{E}-19$ & $2.40 \mathrm{E}-19$ & $1.20 \mathrm{E}-18$ & $7.26 \mathrm{E}-02$ & $1.65 \mathrm{E}-17$ & $1.22 \mathrm{E}-04$ & $-1.10 \mathrm{E}-04$ & $6.61 \mathrm{E}-17$ & $-7.24 \mathrm{E}-21$ \\
\hline
\end{tabular}


Table 3. For a Blackhole having $10^{6}$ solar masses.

\begin{tabular}{|c|c|c|c|c|c|c|c|c|c|c|c|}
\hline $\mathrm{r} / \mathrm{R}$ & $\mathrm{f}_{\mathrm{r}} / \mathrm{f}_{\mathrm{R}}$ & $\rho_{\mathrm{r}}$ & $\mathbf{u}_{\mathrm{r}}$ & $\mathrm{p}_{\mathrm{r}}$ & $\mathrm{q}_{\mathrm{r}}$ & $\mathrm{T}_{\mathrm{r}}$ & $\mathbf{s}_{\mathrm{r}}$ & $\mathrm{F}_{\mathrm{r}}$ & $\mathrm{dT}_{\mathrm{r}} / \mathrm{dr}$ & $\mathrm{C}_{\mathrm{r}}=\mathrm{du}_{\mathrm{r}} / \mathrm{dT}_{\mathrm{r}}$ & $\mathrm{du}_{\mathrm{r}} / \mathrm{dr}$ \\
\hline & & $\left(\mathrm{kg} / \mathrm{m}^{4}\right)$ & $\left(\right.$ Joules $\left./ \mathrm{m}^{4}\right)$ & $\begin{array}{c}\text { (Newtons/ } \\
\mathrm{m}^{3} \text { ) }\end{array}$ & $\left(\right.$ Joules $\left./ \mathrm{m}^{4}\right)$ & $(\mathrm{K})$ & $\begin{array}{l}\text { (Joules/ } \\
\left.\left(\mathrm{m}^{4} \mathrm{~K}\right)\right)\end{array}$ & (Newtons) & $(\mathrm{K} / \mathrm{m})$ & $\begin{array}{c}\text { (Joules/ } \\
\left.\mathrm{m}^{4} \mathrm{~K}\right)\end{array}$ & $\left(\right.$ Joules $\left./ \mathrm{m}^{5}\right)$ \\
\hline 0.000001 & $5.21 \mathrm{E}+37$ & $1.39 \mathrm{E}+16$ & $5.00 \mathrm{E}+32$ & $1.25 \mathrm{E}+32$ & $6.25 \mathrm{E}+32$ & $1.01 \mathrm{E}+09$ & $6.19 \mathrm{E}+23$ & $6.36 \mathrm{E}+43$ & $-2.05 E+05$ & $2.48 \mathrm{E}+24$ & $-5.08 \mathrm{E}+29$ \\
\hline 0.03 & $4.82 \mathrm{E}+37$ & $4.77 \mathrm{E}+02$ & $1.71 \mathrm{E}+19$ & $4.28 \mathrm{E}+18$ & $2.14 \mathrm{E}+19$ & $2.05 \mathrm{E}+06$ & $1.05 \mathrm{E}+13$ & $5.89 \mathrm{E}+43$ & $-1.46 \mathrm{E}-02$ & $4.18 \mathrm{E}+13$ & $-6.10 \mathrm{E}+11$ \\
\hline 0.06 & $3.81 \mathrm{E}+37$ & $4.71 \mathrm{E}+01$ & $1.69 \mathrm{E}+18$ & $4.24 \mathrm{E}+17$ & $2.12 \mathrm{E}+18$ & $1.29 \mathrm{E}+06$ & $1.64 \mathrm{E}+12$ & $4.65 \mathrm{E}+43$ & $-5.28 \mathrm{E}-03$ & $6.57 \mathrm{E}+12$ & $-3.47 \mathrm{E}+10$ \\
\hline 0.09 & $2.58 \mathrm{E}+37$ & $9.45 \mathrm{E}+00$ & $3.40 \mathrm{E}+17$ & $8.49 \mathrm{E}+16$ & $4.24 \mathrm{E}+17$ & $9.35 \mathrm{E}+05$ & $4.54 \mathrm{E}+11$ & $3.15 \mathrm{E}+43$ & $-3.10 \mathrm{E}-03$ & $1.82 \mathrm{E}+12$ & $-5.63 \mathrm{E}+09$ \\
\hline 0.12 & $1.49 \mathrm{E}+37$ & $2.31 E+00$ & $8.29 \mathrm{E}+16$ & $2.07 \mathrm{E}+16$ & $1.04 \mathrm{E}+17$ & $7.05 \mathrm{E}+05$ & $1.47 \mathrm{E}+11$ & $1.82 \mathrm{E}+43$ & $-2.19 \mathrm{E}-03$ & $5.88 \mathrm{E}+11$ & $-1.29 \mathrm{E}+09$ \\
\hline 0.15 & $7.39 \mathrm{E}+36$ & $5.84 \mathrm{E}-01$ & $2.10 \mathrm{E}+16$ & $5.25 \mathrm{E}+15$ & $2.63 \mathrm{E}+16$ & $5.36 \mathrm{E}+05$ & $4.90 \mathrm{E}+10$ & $9.02 \mathrm{E}+42$ & $-1.67 \mathrm{E}-03$ & $1.96 \mathrm{E}+11$ & $-3.27 \mathrm{E}+08$ \\
\hline 0.18 & $3.13 \mathrm{E}+36$ & $1.43 \mathrm{E}-01$ & $5.14 \mathrm{E}+15$ & $1.29 \mathrm{E}+15$ & $6.43 \mathrm{E}+15$ & $4.05 \mathrm{E}+05$ & $1.59 \mathrm{E}+10$ & $3.82 \mathrm{E}+42$ & $-1.31 \mathrm{E}-03$ & $6.36 \mathrm{E}+10$ & $-8.35 \mathrm{E}+07$ \\
\hline 0.21 & $1.13 \mathrm{E}+36$ & $3.26 \mathrm{E}-02$ & $1.17 \mathrm{E}+15$ & $2.93 \mathrm{E}+14$ & $1.47 \mathrm{E}+15$ & $3.01 \mathrm{E}+05$ & $4.87 \mathrm{E}+09$ & $1.38 \mathrm{E}+42$ & $-1.03 \mathrm{E}-03$ & $1.95 \mathrm{E}+10$ & $-2.02 \mathrm{E}+07$ \\
\hline 0.24 & $3.50 \mathrm{E}+35$ & $6.77 \mathrm{E}-03$ & $2.43 \mathrm{E}+14$ & $6.08 \mathrm{E}+13$ & $3.04 \mathrm{E}+14$ & $2.20 \mathrm{E}+05$ & $1.38 \mathrm{E}+09$ & $4.28 \mathrm{E}+41$ & $-8.06 \mathrm{E}-04$ & $5.53 \mathrm{E}+09$ & $-4.46 \mathrm{E}+06$ \\
\hline 0.27 & $9.28 \mathrm{E}+34$ & $1.26 \mathrm{E}-03$ & $4.52 \mathrm{E}+13$ & $1.13 \mathrm{E}+13$ & $5.65 \mathrm{E}+13$ & $1.57 \mathrm{E}+05$ & $3.60 \mathrm{E}+08$ & $1.13 \mathrm{E}+41$ & $-6.16 \mathrm{E}-04$ & $1.44 \mathrm{E}+09$ & $-8.88 \mathrm{E}+05$ \\
\hline 0.3 & $2.10 \mathrm{E}+34$ & $2.08 \mathrm{E}-04$ & $7.47 \mathrm{E}+12$ & $1.87 \mathrm{E}+12$ & $9.34 \mathrm{E}+12$ & $1.09 \mathrm{E}+05$ & $8.53 \mathrm{E}+07$ & $2.57 \mathrm{E}+40$ & $-4.60 \mathrm{E}-04$ & $3.41 \mathrm{E}+08$ & $-1.57 \mathrm{E}+05$ \\
\hline 0.5 & $1.94 \mathrm{E}+28$ & $4.14 \mathrm{E}-11$ & $1.49 \mathrm{E}+06$ & $3.72 \mathrm{E}+05$ & $1.86 \mathrm{E}+06$ & $5.00 \mathrm{E}+03$ & $3.72 \mathrm{E}+02$ & $2.37 \mathrm{E}+34$ & $-3.15 \mathrm{E}-05$ & $1.49 \mathrm{E}+03$ & $-4.68 \mathrm{E}-02$ \\
\hline 1 & $1.00 \mathrm{E}+00$ & $2.67 \mathrm{E}-40$ & $9.60 \mathrm{E}-24$ & $2.40 \mathrm{E}-24$ & $1.20 \mathrm{E}-23$ & $7.26 \mathrm{E}-03$ & $1.65 \mathrm{E}-21$ & $1.22 \mathrm{E}+06$ & $-8.69 \mathrm{E}-11$ & $6.61 \mathrm{E}-21$ & $-5.74 \mathrm{E}-31$ \\
\hline
\end{tabular}

tabulated, as this is simply the sum of columns, 4,5 and 6 . Or, what is equivalent, we could take the entries under column, 3 , for, $\rho_{r}$, in the tables, and, multiply those by $c^{2}$ to obtain $e_{r}$.

In step 6, we finally evaluate the temperature, $T_{r}$. This step is relatively easy. Instead of using Equation, (4-1), we will use a much simpler expression, Equation (2-17). Solving Equation, (2-17), for temperature, $T_{r}$, gives

$$
T_{r}=\left(u_{r} / a^{(4)}\right)^{1 / 5}
$$

In this equation, $a^{(4)}=4.7481 \times 10^{-13}$, in MKS units, as specified in Equation, (2-17). Using the $u_{r}$ values specified in the tables, given under column, 4 , we can thus evaluate the corresponding temperatures by use of Equation, (4-19). These temperatures are listed under column, 7 , in each of our three tables. When comparing the temperatures at the very core for the three black holes considered, we see something very interesting. The temperature actually goes down at the core as the black hole increases its mass. But then the surface temperature just inside the event horizon, $T_{1}$, also decreases its temperature as radius, $R$, increases in value. This would mean that the smallest black holes have the highest temperatures at their core. This fact could have important ramifications for high energy physics, and perhaps even modeling massive particles as mini black holes. 
It also shows that, for a dynamic black hole, the inside surface temperatures will decrease as the radius expands. Assume that a black hole expands from a mass of one solar radius to a mass of ten times that value. Depending on the rate of inflow, this will occur within a certain period of time. Due to the Schwarzschild condition, the radius also increases ten-fold. We are in effect going from Table 1 and Table 2. Now look under the temperature column. We see that not only has the surface temperature just inside the event horizon decreased, but also the core temperature. Think of a dynamic black hole as a series of snapshots of isolated, static black holes, each with an increased radius.

Retracing our steps, we should recognize that, $u_{r}, q_{r}, p_{r}, e_{r}$, are all proportional to one another, and thus to $\rho_{r}$. Using the proportionalities, we can claim that,

$$
u_{r} / u_{R}=q_{r} / q_{R}=p_{r} / p_{R}=e_{r} / e_{R}=\rho_{r} / \rho_{R}=\left(T_{r} / T_{R}\right)^{5}
$$

By setting up a ratio, the constants relating these individual quantities factor out. For the final equality, in Equations, (4-20), we employed the relation, (2-17). Moreover, the mass density, $\rho_{r}$, in turn, is related to $\rho_{R}$, through Equation, (3-18). Because of Equation, (3-18), we can further write,

$$
\begin{aligned}
u_{r} A_{r} /\left(u_{R} A_{R}\right) & =q_{r} A_{r} /\left(q_{R} A_{R}\right)=p_{r} A_{r} /\left(p_{R} A_{R}\right)=e_{r} A_{r} /\left(e_{R} A_{R}\right) \\
& =\rho_{r} A_{r} /\left(\rho_{R} A_{R}\right)=f_{r} / f_{R}=\exp \left[\left(R^{2}-r^{2}\right) / 2 \sigma^{2}\right]
\end{aligned}
$$

An equivalent way of writing this equation is in terms of radii. Because, $A_{r}=2 \pi^{2} r^{3}$, and $A_{R}=2 \pi^{2} R^{3}$, it is possible to write,

$$
\begin{aligned}
u_{r} r^{3} /\left(u_{R} R^{3}\right) & =q_{r} r^{3} /\left(q_{R} R^{3}\right)=p_{r} r^{3} /\left(p_{R} R^{3}\right)=e_{r} r^{3} /\left(e_{R} R^{3}\right) \\
& =\rho_{r} r^{3} /\left(\rho_{R} R^{3}\right)=f_{r} / f_{R}=\exp \left[\left(R^{2}-r^{2}\right) / 2 \sigma^{2}\right]
\end{aligned}
$$

Moreover, we recognize that, by Equation, (2-23), the radiative force at radius, $r$, is given by, $F_{r}=p_{r} A_{r}$. From this, it follows that, $F_{R}=p_{R} A_{R}$. The $3^{\text {rd }}$ equality in Equation, (4-21), can therefore be re-expressed as,

$$
F_{r} / F_{R}=f_{r} / f_{R}=\exp \left[\left(R^{2}-r^{2}\right) / 2 \sigma^{2}\right]
$$

We see from Equations, (4-21), (4-22), and (4-23), that all quantities on the left hand side, are, in effect, determined by the ratio, $f_{r} / f_{R}$. The numerical values for $f_{r} / f_{R}$ are specified in the $2^{\text {nd }}$ column in each of the tables. Again, this specific ratio is fixed by our choice of pdf.

The temperature is also related to this ratio, $f_{r} / f_{R}$. The temperature profile within the black hole, is found using the internal energy density, by Equations, (2-17), or, what is equivalent, (4-19). Using the version indicated by Equation, (4-19), and combining this with the first equality in Equations, (4-20), we are justified in writing,

$$
T_{r} / T_{R}=\left(u_{r} / u_{R}\right)^{1 / 5}=\left(\rho_{r} / \rho_{R}\right)^{1 / 5}
$$

Moreover, multiplying both left and right hand sides by, $(r / R)^{3 / 5}$, we find 
that,

$$
\left(T_{r} / T_{R}\right)(r / R)^{3 / 5}=\left[\rho_{r} r^{3} /\left(\rho_{R} R^{3}\right)\right]^{1 / 5}=\left(f_{r} / f_{R}\right)^{1 / 5}=\exp \left[\left(R^{2}-r^{2}\right) / 10 \sigma^{2}\right]
$$

The last two equalities in Equation, (2-22), were utilized in obtaining the results in Equations, (4-25). We notice from relation, (4-25), that

$$
\left(T_{r} / T_{R}\right)=(R / r)^{3 / 5}\left(f_{r} / f_{R}\right)^{1 / 5}
$$

Equations, (4-24), (4-25), and, (4-26), are some other ways to calculate the temperature, $T_{r}$, within the black hole. However, Equation, (4-19), seems to us to be the most direct way to evaluate these temperatures.

The seventh step is to evaluate the 4-D entropy density function, $s_{r}=s(r)$. This is considered a state variable, i.e., it will depend on the state of the system, defined as a particular layer of the black hole, at a specific temperature. The easiest way to find, $s_{r}$, is to use the last equation, in relations, (2-18). We know the heat density, $q_{r}$, as well as temperature within a specific layer, $T_{r}$. These values were tabulated under columns, 6 , and 7 , respectively. Thus we can find, $s_{r}$, by simply dividing the entry in one column, column 6 , by the corresponding entry in the other, column 7 . The results for, $s_{r}$, are listed under column 8 , in Tables 1-3.

An interesting formulation for entropy density is to use a ratio. We can compare the entropy density within the interior of the black hole, $s_{r}$, to its value just inside the event horizon, where $s_{r}=s_{R}$. In step 2, we evaluated,

$\left(s_{R, A}, s_{R, B}, s_{R, C}\right)=\left(s_{1, A}, s_{1, B}, s_{1, C}\right)$. See Equation, (4-14). We find, upon constructing the ratio,

$$
\begin{aligned}
s_{r} / s_{R} & =\left(q_{r} / q_{R}\right)\left(T_{R} / T_{r}\right)=(R / r)^{3}\left(f_{r} / f_{R}\right)\left(T_{R} / T_{r}\right) \\
& =(R / r)^{3}\left(f_{r} / f_{R}\right)(R / r)^{-3 / 5}\left(f_{r} / f_{R}\right)^{-1 / 5} \\
& =(R / r)^{12 / 5}\left(f_{r} / f_{R}\right)^{4 / 5} \\
& =(R / r)^{12 / 5} \exp \left[0.4\left(R^{2}-r^{2}\right) / \sigma^{2}\right]
\end{aligned}
$$

For the $1^{\text {st }}$ line, we used the last relation in Equations, $(2-18)$, and the $2^{\text {nd }}$ equality in Equations, (4-22). For the second line, Equation, (4-26), was employed. The $3^{\text {rd }}$ line involves simplification. And, as for the $4^{\text {th }}$ line, the last equality, in Equation, (4-21), was utilized. From the last line in relations, (4-27), we see that the entropy density increases rather dramatically if one decreases the radius, $r$. As stated, the values for, $s_{r}$, are specified in the tables. They are to be found under column, 8 .

In step 9, we calculate the 4-D surface area the 4-D radiative force, $F_{r}=p_{r} A_{r}$. These values depend on the radius, and also, on the temperature at radius, $r$. The evaluation of surface area is easy; we simply use $A_{r}=2 \pi^{2} r^{3}=2 \pi^{2} R^{3}(r / R)^{3}$. These values are not tabulated. For, $F_{r}=p_{r} A_{r}$, we multiply the radiative pressure, entered under column 5 , by the corresponding surface area. Those results are tabulated under column, 9 , in the three tables.

As was done for entropy density, we can construct a ratio, which will tell us 
how the radiative force increases within the interior of the black hole. But this was already done by way of Equation, (4-23). Again, the factor, $f_{r} / f_{R}$, comes into play, and this factor is listed under the $2^{\text {nd }}$ column in all three tables. Therefore, because, $F_{r} / F_{R}=f_{r} / f_{R}$, we need only look under the $2^{\text {nd }}$ column in the three tables, to see how radiative force increases as one penetrates into the interior. Needless to say, the radiative force increases dramatically with decreasing $r$ value. Although ridiculously weak at the surface of the black hole, its value within the interior is strong. We can think of the black hole as a multi-layered, 4-D capacitor, where energy densities, radiative forces, and radiative pressures increase substantially with decreasing radii.

In step 10 , we determine the temperature gradient, $\mathrm{d} T_{r} / \mathrm{d} r$, within the $4-\mathrm{D}$ black hole. For this, we will utilize Equation, (4-25), the first and the last entry. Written out somewhat differently, we have

$$
T_{r}^{5} r^{3}=T_{R}^{5} R^{3} \exp \left[\left(R^{2}-r^{2}\right) / 10 \sigma^{2}\right]
$$

We differentiate both the left, and the right, hand sides with respect to radius, $r$. The result is

$$
5 T_{r}^{4} \mathrm{~d} T_{r} / \mathrm{d} r r^{3}+T_{r}^{5} 3 r^{2}=T_{R}^{5} R^{3} \exp \left[\left(R^{2}-r^{2}\right) / 10 \sigma^{2}\right]\left(-r / \sigma^{2}\right)
$$

We next divide the left hand side of Equation, (4-29), by the left hand side of Equation, (4-28). We do the same with the right hand side; divide the right hand side of Equation, (4-29), by the right hand side of Equation, (4-28). In this way we obtain,

$$
\left(5 / T_{r}\right) \mathrm{d} T_{r} / \mathrm{d} r+3 / r=\left(-r / \sigma^{2}\right)
$$

Hence, it follows that,

$$
\mathrm{d} T_{r} / \mathrm{d} r=-\left(r / \sigma^{2}+3 / r\right) T_{r} / 5
$$

The negative sign tells us that we will have a decrease in temperature, in the amount of, $\mathrm{d} T_{r}$, for a corresponding increase in radius, from $r$ to $r+\mathrm{d} r$. This decrease depends, of course, on the shape parameter, $\sigma$, as well as on the radius, $r$, and temperature, $T_{r}$.

We know the values on the right hand side of Equation, (4-31). The radius is specified under column, 1 , in all tables. We use, $r=(r / R) R$. For the temperatures, $T_{r}$, we utilize the corresponding entries under column 7 in all three tables. And the shape parameters are listed in Equations, (3-20). Thus, the right hand side of Equation, (4-31), can be evaluated. We list, $\mathrm{d} T_{r} / \mathrm{d} r$, under column, 10 , in Tables 1-3.

For step, 11, we calculate the 4-D blackbody specific heat, $C_{r} \equiv \mathrm{d} u_{r} / \mathrm{d} T_{r}$, at radius, $r$. Using Equation, (2-17), we see that,

$$
C_{r} \equiv \mathrm{d} u_{r} / \mathrm{d} T_{r}=5 a^{(4)} T_{r}^{4}
$$

However, we also know from the last two equalities in relations, (2-18), that it 
is permissible to write,

$$
s_{r}=5 / 4 u_{r} / T_{r}=5 / 4 a^{(4)} T_{r}^{4}
$$

We utilized Equation, (2-17), for the last equality. Upon comparing Equations, (4-32), with Equation, (4-33), it is obvious that,

$$
C_{r}=4 s_{r}
$$

This very simple result makes it easy to calculate the specific heat, or, $\mathrm{d} u_{r} / \mathrm{d} T_{r}$. We simply take the entries for, $s_{r}$, specified under column 8 in the tables, and multiply them by a factor of 4 . Those values are listed under column 11 in all tables.

Our final step, in this section, is step, 12. Here, we calculate the internal energy density gradient, $\mathrm{d} u_{r} / \mathrm{d} r$. We'll use the fact that,

$$
\mathrm{d} u_{r} / \mathrm{d} r=\left(\mathrm{d} u_{r} / \mathrm{d} T_{r}\right)\left(\mathrm{d} T_{r} / \mathrm{d} r\right)=C_{r}\left(\mathrm{~d} T_{r} / \mathrm{d} r\right)
$$

The entries for, $C_{r}$, are given under column 11 in Tables 1-3. We also know the corresponding values for, $\mathrm{d} T_{r} / \mathrm{d} r$, as these are listed under column 10 . Thus, we are able to evaluate the right hand side of Equation, (4-35). We display those values, in our final column, column 12, in all tables. We remark that because, $\mathrm{d} T_{r} / \mathrm{d} r$, is negative, $\mathrm{d} u_{r} / \mathrm{d} r$, will also be negative. Therefore, for an increase in 4-D radius, from radius, $r$, to radius, $r+\mathrm{d} r$, we expect a decrease in internal energy density, $\mathrm{d} u_{r}$, at a specific radius, $r$. Not only that, but the radiative pressure, the heat density, and the radiative mass density, will also decrease. Refer to Equations, (4-20), where this can be seen directly.

\section{Determining Radiative Mass, Work Done against Radiative Pressure, Gravitational Forces, and Entropy within the Black Hole}

In this section, we will use the two stellar-like equations, which have been generalized to four spatial dimensions, in Section 2. We wish to calculate some important global attributes which hold within, and on the surface, of the black hole. By doing so, we will appreciate the role that the chosen pdf plays in the determination of these physical characteristics. We are looking specifically, at the radiative mass, the work needed to assemble the black hole against radiative pressure, the gravitational forces both within, and on the surface of the black hole, and the entropy. To keep the discussion simple, we will consider only an isolated, static black hole, where there is no net radiative inflow. Net inflow can be considered in a follow up paper. We will also assume that the radiative temperature just outside the event horizon, on the 3-D side, is the CMB temperature, $2.725 \mathrm{~K}$.

As in the previous section, we will proceed in steps, as it will help us to organize our thoughts in a coherent way. Also, we will dispense with the superscripts as all quantities, because, unless otherwise indicated, the quantities are 4-D. The three black holes under consideration are the same as before. Black hole, $B H_{A}$, 
will have a mass equal to that of the sun. Black holes, $B H_{B}$ and, $B H_{C}$, have masses, ten and a million times that of the sun, respectively. Finally, because these global quantities or attributes are dependent on radius, we will have to present our results in table form. We will have three tables; Table 4 refers to black hole, $B H_{A}$, Table 5 to black hole, $B H_{B}$, and Table 6 relates to black hole, $\mathrm{BH}_{\mathrm{C}}$.

We start with the cumulative distribution function (cdf), given by Equation, (3-2). The defining relation, for, $F_{r}^{C D F}$, is Equation, (2-12). Written out once more, we have

$$
F_{r}^{C D F} \equiv \int_{0}^{r} f_{r} \mathrm{~d} r=\operatorname{erf}(r / \sqrt{2} \sigma) / \operatorname{erf}(R / \sqrt{2} \sigma)
$$

For each of the black holes under consideration, black holes $B H_{A}, B H_{B}$, and, $B H_{C}$, we have a specific $R$, and, a specific $\sigma$ value. These are indicated in Equations, (3-18) and (3-20). Therefore, it is possible to evaluate, numerically, the right hand side of Equation, (5-1), for various $r=(r / R) R$ values. These $F_{r}^{C D F}$ values are listed under column 2, in Tables 4-6. Under column 1, we list the internal radii, in steps of, $r=0,0.03 R, 0.06 R, 0.09 R$, etc.. This is step 1 . All tables are presented at the end of the paper.

Step 2 will determine the radiative mass, $M_{r}$, starting from $r=0$, up to, and including radius, $r$. From Equation, (2-11), we know that,

$$
M_{r}=M_{R} F_{r}^{C D F}
$$

\begin{tabular}{|c|c|c|c|c|c|c|c|c|c|c|c|}
\hline $\mathbf{r} / \mathbf{R}$ & $\mathrm{F}^{\mathrm{CDF}}{ }_{\mathrm{r}}$ & Mass $=\mathbf{M}_{\mathrm{r}}$ & $\mathrm{U}_{\mathrm{r}}$ & $\mathrm{Q}_{\mathrm{r}}$ & $\mathrm{W}_{\mathrm{r}}$ & $\mathrm{G}_{\mathrm{r}} \mathrm{M}_{\mathrm{r}}$ & $\mathrm{G}_{\mathrm{r}}$ & $\mathrm{g}_{\mathrm{r}}$ & $\varphi_{\mathrm{G}, \mathrm{r}}$ & $\mathbf{F}_{\mathrm{G}, \mathrm{r}}$ & $\mathrm{S}_{\mathrm{r}}$ \\
\hline & & $(\mathrm{kg})$ & (Joules) & (Joules) & (Joules) & $\left(\mathrm{N} \mathrm{m}^{3} / \mathrm{kg}\right)$ & $\left(\mathrm{N} \mathrm{m}^{3} / \mathrm{kg}^{2}\right)$ & $\left(\mathrm{m} / \mathrm{s}^{2}\right)$ & (Joules/kg) & (Newtons) & (Joules/K) \\
\hline 0.000001 & $1.21 \mathrm{E}-05$ & $2.40 \mathrm{E}+25$ & $8.64 \mathrm{E}+41$ & $1.08 \mathrm{E}+42$ & $2.16 \mathrm{E}+41$ & $1.80 \mathrm{E}+01$ & $7.48 \mathrm{E}-25$ & $-6.97 \mathrm{E}+08$ & $-1.08 \mathrm{E}+18$ & $8.37 \mathrm{E}+33$ & $1.63 \mathrm{E}+29$ \\
\hline 0.03 & $3.50 \mathrm{E}-01$ & $6.97 \mathrm{E}+29$ & $2.50 \mathrm{E}+46$ & $3.13 \mathrm{E}+46$ & $6.26 \mathrm{E}+45$ & $1.46 \mathrm{E}+19$ & $2.09 \mathrm{E}-11$ & $-2.09 \mathrm{E}+13$ & $-1.07 \mathrm{E}+18$ & $7.16 \mathrm{E}+42$ & $2.29 \mathrm{E}+36$ \\
\hline 0.06 & $6.36 \mathrm{E}-01$ & $1.27 \mathrm{E}+30$ & $4.55 \mathrm{E}+46$ & $5.69 \mathrm{E}+46$ & $1.14 \mathrm{E}+46$ & $2.33 \mathrm{E}+20$ & $1.84 \mathrm{E}-10$ & $-4.18 \mathrm{E}+13$ & $-1.07 \mathrm{E}+18$ & $2.47 \mathrm{E}+43$ & $6.25 \mathrm{E}+36$ \\
\hline 0.09 & 8.27E-01 & $1.64 \mathrm{E}+30$ & $5.91 \mathrm{E}+46$ & $7.39 \mathrm{E}+46$ & $1.48 \mathrm{E}+46$ & $1.18 \mathrm{E}+21$ & 7.17E-10 & $-6.28 \mathrm{E}+13$ & $-1.07 \mathrm{E}+18$ & $4.42 \mathrm{E}+43$ & $1.01 \mathrm{E}+37$ \\
\hline 0.12 & $9.31 \mathrm{E}-01$ & $1.85 \mathrm{E}+30$ & $6.66 \mathrm{E}+46$ & $8.32 \mathrm{E}+46$ & $1.66 \mathrm{E}+46$ & $3.73 \mathrm{E}+21$ & $2.01 \mathrm{E}-09$ & $-8.37 \mathrm{E}+13$ & $-1.06 \mathrm{E}+18$ & $5.90 \mathrm{E}+43$ & $1.31 \mathrm{E}+37$ \\
\hline 0.15 & $9.77 \mathrm{E}-01$ & $1.94 \mathrm{E}+30$ & $6.99 \mathrm{E}+46$ & $8.73 \mathrm{E}+46$ & $1.75 \mathrm{E}+46$ & $9.10 \mathrm{E}+21$ & $4.68 \mathrm{E}-09$ & $-1.05 \mathrm{E}+14$ & $-1.05 \mathrm{E}+18$ & $6.75 \mathrm{E}+43$ & $1.48 \mathrm{E}+37$ \\
\hline 0.18 & $9.94 \mathrm{E}-01$ & $1.98 \mathrm{E}+30$ & $7.10 \mathrm{E}+46$ & $8.88 \mathrm{E}+46$ & $1.78 \mathrm{E}+46$ & $1.89 \mathrm{E}+22$ & $9.55 \mathrm{E}-09$ & $-1.26 \mathrm{E}+14$ & $-1.04 \mathrm{E}+18$ & $7.13 \mathrm{E}+43$ & $1.57 \mathrm{E}+37$ \\
\hline 0.21 & $9.99 \mathrm{E}-01$ & $1.99 \mathrm{E}+30$ & $7.14 \mathrm{E}+46$ & $8.92 \mathrm{E}+46$ & $1.78 \mathrm{E}+46$ & $3.50 \mathrm{E}+22$ & $1.76 \mathrm{E}-08$ & $-1.46 \mathrm{E}+14$ & $-1.03 \mathrm{E}+18$ & $7.26 \mathrm{E}+43$ & $1.61 \mathrm{E}+37$ \\
\hline 0.24 & $1.00 \mathrm{E}+00$ & $1.99 \mathrm{E}+30$ & $7.15 \mathrm{E}+46$ & $8.94 \mathrm{E}+46$ & $1.79 \mathrm{E}+46$ & $5.96 \mathrm{E}+22$ & $3.00 \mathrm{E}-08$ & $-1.67 \mathrm{E}+14$ & $-1.02 \mathrm{E}+18$ & $7.30 \mathrm{E}+43$ & $1.62 \mathrm{E}+37$ \\
\hline 0.27 & $1.00 \mathrm{E}+00$ & $1.99 \mathrm{E}+30$ & $7.15 \mathrm{E}+46$ & $8.94 \mathrm{E}+46$ & $1.79 \mathrm{E}+46$ & $9.55 \mathrm{E}+22$ & $4.80 \mathrm{E}-08$ & $-1.88 \mathrm{E}+14$ & $-1.00 \mathrm{E}+18$ & $7.30 \mathrm{E}+43$ & $1.63 \mathrm{E}+37$ \\
\hline 0.3 & $1.00 \mathrm{E}+00$ & $1.99 \mathrm{E}+30$ & $7.15 \mathrm{E}+46$ & $8.94 \mathrm{E}+46$ & $1.79 \mathrm{E}+46$ & $1.46 \mathrm{E}+23$ & $7.32 \mathrm{E}-08$ & $-2.09 \mathrm{E}+14$ & $-9.82 \mathrm{E}+17$ & $7.30 \mathrm{E}+43$ & $1.63 \mathrm{E}+37$ \\
\hline 0.5 & $1.00 \mathrm{E}+00$ & $1.99 \mathrm{E}+30$ & $7.15 \mathrm{E}+46$ & $8.94 \mathrm{E}+46$ & $1.79 \mathrm{E}+46$ & $1.12 \mathrm{E}+24$ & $5.65 \mathrm{E}-07$ & $-3.49 \mathrm{E}+14$ & $-8.18 \mathrm{E}+17$ & $7.30 \mathrm{E}+43$ & $1.63 \mathrm{E}+37$ \\
\hline 1 & $1.00 \mathrm{E}+00$ & $1.99 \mathrm{E}+30$ & $7.15 \mathrm{E}+46$ & $8.94 \mathrm{E}+46$ & $1.79 \mathrm{E}+46$ & $1.80 \mathrm{E}+25$ & $9.04 \mathrm{E}-06$ & $-6.97 \mathrm{E}+14$ & $-4.49 \mathrm{E}+16$ & $7.30 \mathrm{E}+43$ & $1.63 \mathrm{E}+37$ \\
\hline
\end{tabular}

Table 4. For a blackhole having 1 solar mass. 
Table 5. For a blackhole having 10 solar masses.

\begin{tabular}{|c|c|c|c|c|c|c|c|c|c|c|c|}
\hline$r / R$ & $\mathrm{~F}_{\mathrm{r}}^{\mathrm{CDF}}$ & Mass $=\mathbf{M}_{\mathrm{r}}$ & $\mathrm{U}_{\mathrm{r}}$ & $\mathrm{Q}_{\mathrm{r}}$ & $\mathrm{W}_{\mathrm{r}}$ & $\mathrm{G}_{\mathrm{r}} \mathrm{M}_{\mathrm{r}}$ & $\mathrm{G}_{\mathrm{r}}$ & $\mathrm{g}_{\mathrm{r}}$ & $\varphi_{\mathrm{G}, \mathrm{r}}$ & $\mathbf{F}_{\mathrm{G}, \mathrm{r}}$ & $S_{r}$ \\
\hline & & $(\mathrm{kg})$ & (Joules) & (Joules) & (Joules) & $\left(\mathrm{N} \mathrm{m}^{3} / \mathrm{kg}\right)$ & $\left(\mathrm{N} \mathrm{m}^{3} / \mathrm{kg}^{2}\right)$ & $\left(\mathrm{m} / \mathrm{s}^{2}\right)$ & (Joules/kg) & (Newtons) & (Joules/K) \\
\hline 0.000001 & $1.18 \mathrm{E}-05$ & $2.35 E+26$ & $8.46 \mathrm{E}+42$ & $1.06 \mathrm{E}+43$ & $2.12 \mathrm{E}+42$ & $1.73 \mathrm{E}+03$ & $7.33 \mathrm{E}-24$ & $-6.69 \mathrm{E}+07$ & $-1.03 \mathrm{E}+18$ & $7.87 \mathrm{E}+35$ & $6.38 \mathrm{E}+32$ \\
\hline 0.03 & $3.44 \mathrm{E}-01$ & $6.84 \mathrm{E}+30$ & $2.46 \mathrm{E}+47$ & $3.07 \mathrm{E}+47$ & $6.14 \mathrm{E}+46$ & $1.40 \mathrm{E}+21$ & $2.04 \mathrm{E}-10$ & $-2.01 \mathrm{E}+12$ & $-1.03 \mathrm{E}+18$ & $6.74 \mathrm{E}+44$ & $8.98 \mathrm{E}+39$ \\
\hline 0.06 & $6.26 \mathrm{E}-01$ & $1.25 \mathrm{E}+31$ & $4.48 \mathrm{E}+47$ & $5.60 \mathrm{E}+47$ & $1.12 \mathrm{E}+47$ & $2.24 \mathrm{E}+22$ & $1.79 \mathrm{E}-09$ & $-4.02 \mathrm{E}+12$ & $-1.03 \mathrm{E}+18$ & $2.34 \mathrm{E}+45$ & $2.46 \mathrm{E}+40$ \\
\hline 0.09 & $8.18 \mathrm{E}-01$ & $1.63 \mathrm{E}+31$ & $5.85 \mathrm{E}+47$ & $7.31 \mathrm{E}+47$ & $1.46 \mathrm{E}+47$ & $1.13 \mathrm{E}+23$ & $6.96 \mathrm{E}-09$ & $-6.02 E+12$ & $-1.03 \mathrm{E}+18$ & $4.22 \mathrm{E}+45$ & $4.02 \mathrm{E}+40$ \\
\hline 0.12 & $9.25 \mathrm{E}-01$ & $1.84 \mathrm{E}+31$ & $6.61 \mathrm{E}+47$ & $8.27 \mathrm{E}+47$ & $1.65 \mathrm{E}+47$ & $3.58 \mathrm{E}+23$ & $1.94 \mathrm{E}-08$ & $-8.03 E+12$ & $-1.02 \mathrm{E}+18$ & $5.69 \mathrm{E}+45$ & $5.21 \mathrm{E}+40$ \\
\hline 0.15 & $9.74 \mathrm{E}-01$ & $1.94 \mathrm{E}+31$ & $6.96 \mathrm{E}+47$ & $8.70 \mathrm{E}+47$ & $1.74 \mathrm{E}+47$ & $8.73 \mathrm{E}+23$ & $4.51 \mathrm{E}-08$ & $-1.00 \mathrm{E}+13$ & $-1.01 \mathrm{E}+18$ & $6.55 \mathrm{E}+45$ & $5.95 \mathrm{E}+40$ \\
\hline 0.18 & $9.92 \mathrm{E}-01$ & $1.97 \mathrm{E}+31$ & $7.10 \mathrm{E}+47$ & $8.87 \mathrm{E}+47$ & $1.77 \mathrm{E}+47$ & $1.81 \mathrm{E}+24$ & $9.18 \mathrm{E}-08$ & $-1.20 \mathrm{E}+13$ & $-1.00 \mathrm{E}+18$ & $6.95 \mathrm{E}+45$ & $6.34 \mathrm{E}+40$ \\
\hline 0.21 & $9.98 \mathrm{E}-01$ & $1.99 \mathrm{E}+31$ & $7.14 \mathrm{E}+47$ & $8.92 \mathrm{E}+47$ & $1.78 \mathrm{E}+47$ & $3.36 \mathrm{E}+24$ & $1.69 \mathrm{E}-07$ & $-1.41 \mathrm{E}+13$ & $-9.90 \mathrm{E}+17$ & $7.10 \mathrm{E}+45$ & $6.50 \mathrm{E}+40$ \\
\hline 0.24 & $1.00 \mathrm{E}+00$ & $1.99 \mathrm{E}+31$ & $7.15 \mathrm{E}+47$ & $8.93 \mathrm{E}+47$ & $1.79 \mathrm{E}+47$ & $5.72 \mathrm{E}+24$ & $2.88 \mathrm{E}-07$ & $-1.61 \mathrm{E}+13$ & $-9.77 \mathrm{E}+17$ & $7.14 \mathrm{E}+45$ & $6.56 \mathrm{E}+40$ \\
\hline 0.27 & $1.00 \mathrm{E}+00$ & $1.99 \mathrm{E}+31$ & $7.15 \mathrm{E}+47$ & $8.94 \mathrm{E}+47$ & $1.79 \mathrm{E}+47$ & $9.17 \mathrm{E}+24$ & 4.61E-07 & $-1.81 \mathrm{E}+13$ & $-9.61 \mathrm{E}+17$ & $7.15 \mathrm{E}+45$ & $6.58 \mathrm{E}+40$ \\
\hline 0.3 & $1.00 \mathrm{E}+00$ & $1.99 \mathrm{E}+31$ & $7.15 \mathrm{E}+47$ & $8.94 \mathrm{E}+47$ & $1.79 \mathrm{E}+47$ & $1.40 \mathrm{E}+25$ & 7.03E-07 & $-2.01 \mathrm{E}+13$ & $-9.45 \mathrm{E}+17$ & $7.16 \mathrm{E}+45$ & $6.59 \mathrm{E}+40$ \\
\hline 0.5 & $1.00 \mathrm{E}+00$ & $1.99 \mathrm{E}+31$ & $7.15 \mathrm{E}+47$ & $8.94 \mathrm{E}+47$ & $1.79 \mathrm{E}+47$ & $1.08 \mathrm{E}+26$ & $5.42 \mathrm{E}-06$ & $-3.35 E+13$ & $-7.86 \mathrm{E}+17$ & $7.16 \mathrm{E}+45$ & $6.59 \mathrm{E}+40$ \\
\hline 1 & $1.00 \mathrm{E}+00$ & $1.99 \mathrm{E}+31$ & $7.15 \mathrm{E}+47$ & $8.94 \mathrm{E}+47$ & $1.79 \mathrm{E}+47$ & $1.73 \mathrm{E}+27$ & 8.67E-05 & $-6.69 \mathrm{E}+13$ & $-4.49 \mathrm{E}+16$ & $7.16 \mathrm{E}+45$ & $6.59 \mathrm{E}+40$ \\
\hline
\end{tabular}

Table 6. For a blackhole having $10^{6}$ solar masses.

\begin{tabular}{|c|c|c|c|c|c|c|c|c|c|c|c|}
\hline $\mathbf{r} / \mathbf{R}$ & $\mathrm{F}^{\mathrm{CDF}}{ }_{\mathrm{r}}$ & Mass $=M_{r}$ & $\mathrm{U}_{\mathrm{r}}$ & $\mathrm{Q}_{\mathrm{r}}$ & $\mathrm{W}_{\mathrm{r}}$ & $\mathrm{G}_{\mathrm{r}} \mathrm{M}_{\mathrm{r}}$ & $\mathrm{G}_{\mathrm{r}}$ & $\mathrm{g}_{\mathrm{r}}$ & $\varphi_{\mathrm{G}, \mathrm{r}}$ & $\mathbf{F}_{\mathrm{G}, \mathrm{r}}$ & $\mathrm{S}_{\mathrm{r}}$ \\
\hline & & $(\mathrm{kg})$ & (Joules) & (Joules) & (Joules) & $\left(\mathrm{N} \mathrm{m}^{3} / \mathrm{kg}\right)$ & $\left(\mathrm{N} \mathrm{m}^{3} / \mathrm{kg}^{2}\right)$ & $\left(\mathrm{m} / \mathrm{s}^{2}\right)$ & (Joules/kg) & (Newtons) & (Joules/K) \\
\hline 0.000001 & $1.05 \mathrm{E}-05$ & $2.09 \mathrm{E}+31$ & $7.52 \mathrm{E}+47$ & $9.40 \mathrm{E}+47$ & $1.88 \mathrm{E}+47$ & $1.36 \mathrm{E}+13$ & $6.51 \mathrm{E}-19$ & $-5.28 \mathrm{E}+02$ & $-8.25 \mathrm{E}+17$ & $5.52 \mathrm{E}+33$ & $5.81 \mathrm{E}+38$ \\
\hline 0.03 & $3.07 \mathrm{E}-01$ & $6.11 \mathrm{E}+35$ & $2.20 \mathrm{E}+52$ & $2.75 \mathrm{E}+52$ & $5.50 \mathrm{E}+51$ & $1.10 \mathrm{E}+31$ & $1.80 \mathrm{E}-05$ & $-1.59 \mathrm{E}+07$ & $-8.25 \mathrm{E}+17$ & $4.78 \mathrm{E}+42$ & $8.23 \mathrm{E}+45$ \\
\hline 0.06 & $5.71 \mathrm{E}-01$ & $1.14 \mathrm{E}+36$ & $4.08 \mathrm{E}+52$ & $5.10 \mathrm{E}+52$ & $1.02 \mathrm{E}+52$ & $1.77 \mathrm{E}+32$ & $1.55 \mathrm{E}-04$ & $-3.17 \mathrm{E}+07$ & $-8.23 \mathrm{E}+17$ & $1.71 \mathrm{E}+43$ & $2.30 \mathrm{E}+46$ \\
\hline 0.09 & 7.64E-01 & $1.52 \mathrm{E}+36$ & $5.47 \mathrm{E}+52$ & $6.83 \mathrm{E}+52$ & $1.37 \mathrm{E}+52$ & $8.94 \mathrm{E}+32$ & $5.88 \mathrm{E}-04$ & $-4.76 \mathrm{E}+07$ & $-8.19 \mathrm{E}+17$ & $3.21 \mathrm{E}+43$ & $3.87 \mathrm{E}+46$ \\
\hline 0.12 & $8.86 \mathrm{E}-01$ & $1.76 \mathrm{E}+36$ & $6.34 \mathrm{E}+52$ & $7.92 \mathrm{E}+52$ & $1.58 \mathrm{E}+52$ & $2.82 \mathrm{E}+33$ & $1.60 \mathrm{E}-03$ & $-6.34 \mathrm{E}+07$ & $-8.14 \mathrm{E}+17$ & $4.54 \mathrm{E}+43$ & $5.20 \mathrm{E}+46$ \\
\hline 0.15 & $9.52 \mathrm{E}-01$ & $1.89 \mathrm{E}+36$ & $6.81 \mathrm{E}+52$ & $8.51 \mathrm{E}+52$ & $1.70 \mathrm{E}+52$ & $6.90 \mathrm{E}+33$ & $3.64 \mathrm{E}-03$ & $-7.93 \mathrm{E}+07$ & $-8.08 \mathrm{E}+17$ & $5.46 \mathrm{E}+43$ & $6.15 \mathrm{E}+46$ \\
\hline 0.18 & $9.82 \mathrm{E}-01$ & $1.95 \mathrm{E}+36$ & $7.02 \mathrm{E}+52$ & $8.78 \mathrm{E}+52$ & $1.76 \mathrm{E}+52$ & $1.43 \mathrm{E}+34$ & 7.32E-03 & $-9.51 \mathrm{E}+07$ & $-8.00 \mathrm{E}+17$ & $5.98 \mathrm{E}+43$ & $6.72 \mathrm{E}+46$ \\
\hline 0.21 & $9.94 \mathrm{E}-01$ & $1.98 \mathrm{E}+36$ & $7.11 \mathrm{E}+52$ & $8.89 \mathrm{E}+52$ & $1.78 \mathrm{E}+52$ & $2.65 \mathrm{E}+34$ & $1.34 \mathrm{E}-02$ & $-1.11 \mathrm{E}+08$ & $-7.91 \mathrm{E}+17$ & $6.22 \mathrm{E}+43$ & $7.02 \mathrm{E}+46$ \\
\hline 0.24 & $9.98 \mathrm{E}-01$ & $1.99 \mathrm{E}+36$ & $7.14 \mathrm{E}+52$ & $8.92 \mathrm{E}+52$ & $1.78 \mathrm{E}+52$ & $4.52 \mathrm{E}+34$ & $2.28 \mathrm{E}-02$ & $-1.27 \mathrm{E}+08$ & $-7.81 \mathrm{E}+17$ & $6.32 \mathrm{E}+43$ & $7.16 \mathrm{E}+46$ \\
\hline 0.27 & $1.00 \mathrm{E}+00$ & $1.99 \mathrm{E}+36$ & $7.15 \mathrm{E}+52$ & $8.93 \mathrm{E}+52$ & $1.79 \mathrm{E}+52$ & $7.24 \mathrm{E}+34$ & $3.64 \mathrm{E}-02$ & $-1.43 \mathrm{E}+08$ & $-7.69 \mathrm{E}+17$ & $6.35 \mathrm{E}+43$ & $7.21 \mathrm{E}+46$ \\
\hline 0.3 & $1.00 \mathrm{E}+00$ & $1.99 \mathrm{E}+36$ & $7.15 \mathrm{E}+52$ & $8.94 \mathrm{E}+52$ & $1.79 \mathrm{E}+52$ & $1.10 \mathrm{E}+35$ & $5.55 \mathrm{E}-02$ & $-1.59 \mathrm{E}+08$ & $-7.55 \mathrm{E}+17$ & $6.36 \mathrm{E}+43$ & $7.23 \mathrm{E}+46$ \\
\hline 0.5 & $1.00 \mathrm{E}+00$ & $1.99 \mathrm{E}+36$ & $7.15 \mathrm{E}+52$ & $8.94 \mathrm{E}+52$ & $1.79 \mathrm{E}+52$ & $8.51 E+35$ & $4.28 \mathrm{E}-01$ & $-2.64 \mathrm{E}+08$ & $-6.30 \mathrm{E}+17$ & $6.36 \mathrm{E}+43$ & $7.24 \mathrm{E}+46$ \\
\hline 1 & $1.00 \mathrm{E}+00$ & $1.99 \mathrm{E}+36$ & $7.15 \mathrm{E}+52$ & $8.94 \mathrm{E}+52$ & $1.79 \mathrm{E}+52$ & $1.36 \mathrm{E}+37$ & $6.85 \mathrm{E}+00$ & $-5.28 \mathrm{E}+08$ & $-4.49 \mathrm{E}+16$ & $6.36 \mathrm{E}+43$ & $7.24 \mathrm{E}+46$ \\
\hline
\end{tabular}


Thus, for a determination of, $M_{r}$, all that is needed is for us to multiply the total mass of the black hole, $M_{R}$, by the cdf, specified in the $2^{\text {nd }}$ column, in Tables 4-6. These values are entered in the third column in the corresponding tables. It is to be noticed, upon inspection of the results, that most of the radiative mass is concentrated relatively close to the center of the black hole. This holds for all three black holes considered. This is a consequence of the pdf chosen, a truncated Gaussian, and the fact that the values indicated in Equations, (3-21), are relatively low in value. In other words, the dispersion is low.

In step 3, we seek to find the total radiative energy, $E_{r}$, starting from, $r=0$, up to, and including radius, $r$. We are also interested in finding the total internal energy, $U_{r}$, the total heat trapped, $Q_{r}$, and the total work done against radiative pressure in assembling the black hole, $W_{r}$. The quantities are to be evaluated, starting from, $r=0$, up to, and including radius, $r$. We start with, $E_{r}$. To find the total radiative energy, we integrate over the energy density function, but 4-dimensionally, from, $r=0$, to a final value, $r$. Within an infinitesimally small, 4-D volume element, $\mathrm{d} V_{r}=A_{r} \mathrm{~d} r=2 \pi^{2} r^{3} \mathrm{~d} r$, we will have trapped electromagnetic energy in the amount, $\mathrm{d} E_{r}=e_{r} \mathrm{~d} V_{r}=\rho_{r} c^{2} \mathrm{~d} V_{r}$. This is stored energy in a 4-D layer, between radius, $r$, and radius, $r+\mathrm{d} r$. We integrate to find the total energy contained up to radius, $r$. The result is,

$$
E_{r}=\int_{0}^{r} \mathrm{~d} E_{r}=\int_{0}^{r} e_{r} \mathrm{~d} V_{r}=\int_{0}^{r} \rho_{r} c^{2} \mathrm{~d} V_{r}=M_{r} c^{2}
$$

The mass of the black hole is thought to be made up of radiative energy, in all its forms, internal, plus pressure, plus heat. For the last equality, we made use of Equation, (2-4). If we specialize Equation, (5-3), to $r=R$, we obtain the full mass, $M_{R}$, for the mass of the black hole on the right hand side. See Equation, $(2-5)$. This is the same mass that we observe three-dimensionally.

The next quantity to be determined is the internal energy, $U_{r}$. We follow the same steps as before. Within an infinitesimal volume element, $\mathrm{d} V_{r}=A_{r} \mathrm{~d} r$, we must have internal energy in the amount, $\mathrm{d} U_{r}=u_{r} A_{r} \mathrm{~d} r$. We integrate to find

$$
U_{r}=\int_{0}^{r} \mathrm{~d} U_{r}=\int_{0}^{r} u_{r} \mathrm{~d} V_{r}=\int_{0}^{r} \frac{4}{10} e_{r} \mathrm{~d} V_{r}=\frac{4}{10} M_{r} c^{2}
$$

For the $3^{\text {rd }}$ equality, we recognized that, $u_{r}=\frac{4}{10} e_{r}$. See the last equality in Equations, (2-22). We proceed similarly for the heat trapped, and the work done against radiative pressure. For the former, we find,

$$
Q_{r}=\int_{0}^{r} \mathrm{~d} Q_{r}=\int_{0}^{r} q_{r} \mathrm{~d} V_{r}=\int_{0}^{r} \frac{1}{2} e_{r} \mathrm{~d} V_{r}=\frac{1}{2} M_{r} c^{2}
$$

And for the latter, we obtain,

$$
W_{r}=\int_{0}^{r} \mathrm{~d} W_{r}=\int_{0}^{r} p_{r} \mathrm{~d} V_{r}=\int_{0}^{r} \frac{1}{10} e_{r} \mathrm{~d} V_{r}=\frac{1}{10} M_{r} c^{2}
$$

We made use of the relations in Equations, (2-22), in order to relate heat density, and radiative pressure, to, $e_{r}$. If we specialize the Equations, (5-4), (5-5), 
and (5-6), to $r=R$, we see that,

$$
U_{R}=\frac{4}{10} M_{R} c^{2}, Q_{R}=\frac{1}{2} M_{R} c^{2}, W_{R}=\frac{1}{10} M_{R} c^{2}
$$

The factors of $\frac{4}{10}, \frac{5}{10}$, and, $\frac{1}{10}$, in all these relations remain the same, whether we consider, a layer, an enclosing volume, or the total volume. The factors are imposed upon us by the dimensionality of space and the fact that we are dealing with blackbody radiation, period. What is obvious with these relations is the fact that the internal energy makes up $40 \%$ of the total energy, the trapped heat represents $50 \%$ of the total energy, and the work done against pressure makes up $10 \%$ of the total. In $3-\mathrm{D}$ space, the proportions would be different. In three dimensions, the corresponding proportions would be, $\frac{3}{8}=37.5 \%$, $\frac{4}{8}=50 \%$, and, $\frac{1}{8}=12.5 \%$.

Whenever we integrate pressure over volume, we obtain work done. Equation, (5-6), is particularly noteworthy from that point of view. We interpret this equation as the work done in assembling the black hole against radiative pressure, which will want to blow the black hole out. What keeps this radiative mass held together (contained) must be gravity. Our hydrostatic equation shows that. Layer by layer, gravity holds the radiative forces in check. See Equation, (2-26). In a sense, a black hole is something like a 4-D version of ball lightning, but because of the 4-D space, it is nonluminous. See the waterfall model described in reference [4]. We can think of it as an exotic type of 4-D capacitor, filled with blackbody radiative energy in a multi-layered fashion.

Coming back to our tables, we list $U_{r}, Q_{r}$, and, $W_{r}$, under columns 4,5 and 6 , respectively. In each of our Tables 4-6, we have calculated these values. The total (radiative mass) energy is not tabulated. This can be found most easily, by multiplying, the radiative mass, entered under column 3 , by $c^{2}$. Another way is to sum columns 4,5 , and 6 , which gives the same results.

The next quantity to be considered is the product, $G_{r} M_{r}=G_{r}^{(4)} M_{r}^{(4)}$, which brings us to step 4 . In this step, we introduce the 4-D gravitational "constant", $G_{r}=G_{r}^{(4)}$, which is not really a constant as it will depend on the 4-D radius. It is model dependent, unlike Newton's constant, $G_{N}$. In other words, its value depends on the pdf chosen. To determine the product of, $G_{r} M_{r}$, we will use Equation, (2-30). This can be rewritten in the form,

$$
G_{r} M_{r} \rho_{r}=-\left(\mathrm{d} p_{r} / \mathrm{d} r\right) r^{3}-3 p_{r} r^{2}
$$

On the left hand side of this equation, we express the radiative mass density, $\rho_{r}$, in terms of the radiative pressure, $p_{r}$. From Equation, (2-21), it can be seen that,

$$
\rho_{r} A_{r}=10 p_{r} / c^{2} A_{r}
$$

Moreover, using Equation, (4-21), it is also true that, 


$$
p_{r} A_{r} /\left(p_{R} A_{R}\right)=\exp \left[\left(R^{2}-r^{2}\right) / 2 \sigma^{2}\right]
$$

We differentiate both left and right hand sides of this equation with respect to radius, $r$. The result is

$$
\left[\left(\mathrm{d} p_{r} / \mathrm{d} r\right) A_{r}+p_{r}\left(\mathrm{~d} A_{r} / \mathrm{d} r\right)\right] /\left(p_{R} A_{R}\right)=\exp \left[\left(R^{2}-r^{2}\right) / 2 \sigma^{2}\right]\left(-r / \sigma^{2}\right)
$$

Next, we divide the left hand side of Equation, (5-11), by the left hand side of Equation, (5-10), and we do the same on the right hand side. We obtain in this way, the new result

$$
\left(1 / p_{r}\right)\left(\mathrm{d} p_{r} / \mathrm{d} r\right)+\left(1 / A_{r}\right)\left(\mathrm{d} A_{r} / \mathrm{d} r\right)=\left(-r / \sigma^{2}\right)
$$

However, $\left(1 / A_{r}\right)\left(\mathrm{d} A_{r} / \mathrm{d} r\right)=3 / r$. And therefore, Equation, (5-12), can be rewritten as,

$$
\left(\mathrm{d} p_{r} / \mathrm{d} r\right)=\left(-p_{r}\right)\left(3 / r+r / \sigma^{2}\right)
$$

We next substitute both Equations, (5-9), and, (5-13), into Equation, (5-8). The relation, (5-9), is used to eliminate, $\rho_{r}$, on the left side of Equation, (5-8), whereas relation,(5-13), will eliminate the $\left(\mathrm{d} p_{r} / \mathrm{d} r\right)$ term on the right hand side. In this way, we obtain,

$$
G_{r} M_{r} 10 p_{r} / c^{2}=p_{r}\left(3 / r+r / \sigma^{2}\right) r^{3}-3 p_{r} r^{2}
$$

Two terms cancel on the right hand side, and we divide the remaining expression by, $p_{r}$. After rearranging terms, we find the much simpler result,

$$
G_{r} M_{r}=0.1 c^{2}\left(r^{4} / \sigma^{2}\right)
$$

This is a very interesting expression because it depends on radius, and the shape parameter, $\sigma$.

Now, for the three black holes considered we know the shape parameters. These are given in Equations, (3-20). Therefore, we can evaluate, $G_{r} M_{r}$, as a function of radius, using the above Equation, (5-15). The results are present under column, 7, in each of the three Tables 4-6.

This brings us to step 5. In this step, we calculate, $G_{r}=G_{r}^{(4)}$. From Equation, (2-11), we saw that that the radiative mass, $M_{r}$, at radius, $r$, is proportional to the cumulative distribution function, $F_{r}^{C D F}$, by means of the relation, $M_{r}=F_{r}^{C D F} M_{R}$. Moreover, $M_{R}$, being the total mass of the black hole, can be related to its total radius, $R$, by way of the Schwarzschild relation, $R=2 G M_{R} / c^{2}$. We can therefore write in place of $M_{r}$, the equation, $M_{r}=F_{r}^{C D F} M_{R}=F_{r}^{C D F}\left(R c^{2} / 2 G\right)$. We substitute this for, $M_{r}$ on the left hand side of Equation, (5-15). This gives us,

$$
G_{r} F_{r}^{C D F}\left(R c^{2} / 2 G\right)=0.1 c^{2}\left(r^{4} / \sigma^{2}\right)
$$

Simplifying, and rearranging terms, it follows that,

$$
G_{r} F_{r}^{C D F}=0.2(G / R)\left(r^{4} / \sigma^{2}\right)
$$

This equation shows us the relation between the 4-D gravitational "strength", 
$G_{r}$, and Newton's constant, $G$. If we consider the special case where, $r=R$, this reduces to,

$$
G_{R}=0.2 G\left(R^{3} / \sigma^{2}\right)
$$

At, $r=R$, we have a sharp discontinuity in gravitational strength due to the fact that $0.2\left(R^{3} / \sigma^{2}\right) \neq 1$. In other words the gravitational strength does not match at the event horizon, when we compare its value on the 3-D side versus on the 4-D side. As one enters the 4-D black hole from 3-D space, the gravitational strength will increase abruptly in value, from, $G$, to $G_{R}=G_{R}^{(4)}$. However, as argued in the previous paper [5], this is to be expected. Many physical quantities will change their value abruptly due to the discontinuity of space. Gravity is no exception. We referred to this state of affairs in our previous work as our "waterfall model". We emphasize, once more, that the results indicated by Equations, (5-15), and, (5-18), are pdf dependent. If we had introduced a different pdf, other than the truncated Gaussian, to model the interior, radiative mass, distribution of the black hole, then we would have obtained different expressions for the strength of gravity both within, and on the surface, of the black hole.

We also notice, from Equation, (5-17), that in the limit where, $r \rightarrow 0$, both the left hand side and right hand side vanish. In this limit, $F_{r}^{C D F} \rightarrow 0$, as can be seen from Equation, (2-12). At the very center of the black hole, it would appear as if there is no gravitational strength. In actual fact, it can take on any value in this singular limit, as Equation, (5-17), would still be satisfied. However, as soon as the radius becomes finite, not any value is allowed; Equation, (5-17), determines a specific value for, $G_{r}$. For a given size black hole, where we have a definitive $\sigma$ value, the gravitational strength will increase as, $G_{r} \sim r^{4}$, as indicated by relation, (5-17). For each of the black hole masses considered, we have the corresponding $R$ and $\sigma$ values. See Equations, (3-18), and, (3-20). These values can be substituted in Equation, (5-17), to find $G_{r}$, for various $r$ values. We also need the $F_{r}^{C D F}$ values, but these are specified under column 2, in each of the tables. The calculated $G_{r}$ values, using Equation, (5-17), are listed under column 8, in each of the Tables 4-6.

It would have also been possible to obtain, $G_{r}$, using the product, $G_{r} M_{r}$, specified under column 7, and dividing out the corresponding $M_{r}$ terms, which are indicated under column 3 . However, this approach would not have given us explicit mathematical expressions for, $G_{r}$.

In step 6, we determine the gravitational acceleration, $g_{r}=g_{r}^{(4)}$, within the 4 -D black hole. This is most easily accomplished using a $4-\mathrm{D}$ version of Gauss's law. In 4-D space, we generalize Gauss's law to read,

$$
\oint \boldsymbol{g}_{r} \cdot \mathrm{d} \boldsymbol{A}_{r}=-2 \pi^{2} r^{3} g_{r}=M_{r} / \varepsilon_{r}
$$

The $M_{r}$ stands for the radiative mass which is enclosed within radius, $r$, and we have assumed spherical symmetry. The negative sign is due to $\boldsymbol{g}_{r}$ pointing radially inwards. We have introduced a "gravitational permittivity", $\varepsilon_{r}$, on the right hand side. As we shall see, this quantity will be radius dependent, and 
hence the subscript, $r$. This is the gravitational permittivity to be associated with the photon-packed blackbody medium. The permittivity really refers to the permittivity of a material substance, which, in this instance, is blackbody radiation at a specific temperature. Hence, it is radius dependent.

We rewrite Equation, (5-19), as

$$
g_{r}=g_{r}^{(4)}=-\left(\frac{1}{2 \pi^{2} \varepsilon_{r}}\right) M_{r} / r^{3}=-G_{r} M_{r} / r^{3}
$$

where,

$$
G_{r}=G_{r}^{(4)} \equiv 1 /\left(2 \pi^{2} \varepsilon_{r}\right)
$$

We can think of Equation, (5-21), as the defining equation for $\varepsilon_{r}$. Because, $G_{r}$, is a function of radius, $r$, so too, is, $\varepsilon_{r}$. This makes sense as an energetically denser medium should be reflected in the gravitational permittivity of the medium. Moreover, Equation, (5-20), seems a logical 4-D extension of the familiar 3-D equation, $g^{(3)}=-G M_{r} / r^{2}$.

We next substitute Equation, (5-15), into the right hand side of Equation, (5-20), and cancel terms. We thereby obtain a relatively simple result,

$$
g_{r}=g_{r}^{(4)}=-0.1 c^{2} r / \sigma^{2}=-0.2 G M_{R} / R\left(r / \sigma^{2}\right)
$$

For the second equality, we made use of the Schwarzschild equation. Equation, (5-22), is a very elegant result. It tells us that the gravitational acceleration increases linearly with radius, $r$, within the 4-D black hole of a specified mass, or radius. This is so in spite of the fact that the medium does not have uniform density. Also remember that the mass, or radius, of the black hole will determine its shape parameter, $\sigma$, as was seen in Section 3. As such, $\sigma$, is, effectively, a constant. Equation, (5-22), is specific to the pdf chosen, which, in our situation, is a truncated Normal distribution. At the event horizon where the radius, $r=R$, we see that, $g_{R}=-0.1 c^{2} R / \sigma^{2}$. Equations, (5-20), and, (5-22), are to be noted for their simplicity. A different pdf would have led to different results.

We have calculated the gravitational acceleration, using Equation, (5-22), for the three black holes we are analyzing. The results are presented in Tables 4-6, under column 9. The gravitational acceleration will have its greatest value at the event horizon, much like the Earth.

For step 7, we calculate the gravitational potential, $\varphi_{r}$, as a function of radius, $r$, which also will include, $r=R$. We know that the gravitational acceleration is negative the gradient of the gravitational potential. If we have spherical symmetry, then, $g_{r}=-\mathrm{d} \varphi_{r} / \mathrm{d} r$, where $g_{r}$ is directed radially in. Therefore, $\mathrm{d} \varphi_{r}=-g_{r} \mathrm{~d} r$, and we can integrate. For the gravitational acceleration, $g_{r}$, we will use the first equality in Equation, (5-22). Thus, upon substitution,

$$
\begin{gathered}
\int_{0}^{r} \mathrm{~d} \varphi_{r}=\int_{0}^{r}\left(0.1 c^{2}\right)\left(r / \sigma^{2}\right) \mathrm{d} r \\
\varphi_{r}-\varphi_{0}=\left(0.1 c^{2}\right)\left(r^{2} / 2 \sigma^{2}\right)
\end{gathered}
$$


This can also be rewritten as,

$$
\varphi_{r}-\varphi_{0}=\left(0.2 G M_{R} / R\right)\left(r^{2} / 2 \sigma^{2}\right)
$$

because by the Schwarzschild condition, $c^{2}=2 G M_{R} / R$. At the particular radius, $r=R$, Equations, (5-23), and, (5-24), become,

$$
\varphi_{R}=\varphi_{0}+\left(0.1 c^{2}\right)\left(R^{2} / 2 \sigma^{2}\right)=\varphi_{0}+\left(0.1 G M_{R} R / \sigma^{2}\right)
$$

The value, $\varphi_{0}$, is our constant of integration.

We want the 4-D gravitational potential to match the 3-D gravitational potential at, $r=R$. This will guarantee an equipotential surface for the event horizon, which has exactly the same value in both 3-D and 4-D space. For this purpose, we will demand that, $\varphi_{R}^{(4)}=\varphi_{R}^{(3)}$, at radius, $R$. Using the second expression in Equation, (5-25), we obtain

$$
\varphi_{0}+\left(0.1 G M_{R} R / \sigma^{2}\right)=-G M_{R} / R
$$

This fixes the constant of integration to equal,

$$
\varphi_{0}=-G M_{R} / R\left[1+0.1 R^{2} / \sigma^{2}\right]
$$

Coming back to our original expression, (5-24), we see that this can now be rewritten as,

$$
\varphi_{r}=\left(-G M_{R} / R\right)\left[1+0.1\left(R^{2}-r^{2}\right) / \sigma^{2}\right]
$$

This is our expression for the gravitational potential, within the black hole, and on its surface. We note that when, $r=R$, we retrieve our familiar, 3-D expression for the gravitational potential.

Utilizing Equation, (5-28), we can find $\varphi_{r}$ for any value of radius, $r$. The right hand side can easily be evaluated for a specific black hole mass. Given the black hole radius, or mass, we can also find, $\sigma$, using the methods illustrated in Section 3. We have found the shape parameter for three black holes; they are indicated in Equations, (3-20). For these very specific black holes, we have calculated the gravitational potential as a function of $r$. The values are listed under column 10, in Tables 4-6. The gravitational potential is most negative at the very interior of the black hole.

The next step is step 8. In this step, we will evaluate the gravitational force. This calculation is very significant is that it will allow us to determine the total surface tension, which is needed to find the total work done in expanding the event horizon. See reference [5]. To determine the gravitational force, we will make use of the gravitational acceleration. If we consider a layer of thickness, $\mathrm{d} r$, between radii, $r$, and, $r+\mathrm{d} r$, the gravitational force acting on that layer, must be,

$$
\begin{aligned}
\mathrm{d} F_{G, r} & =\left(g_{r}\right)\left(\mathrm{d} M_{r}\right)=\left(g_{r}\right)\left(\rho_{r} \mathrm{~d} V_{r}\right) \\
& =\left(g_{r}\right)\left(\rho_{r} A_{r} \mathrm{~d} r\right)=\left(-0.1 c^{2} r / \sigma^{2}\right)\left(M_{R} f_{r} \mathrm{~d} r\right)
\end{aligned}
$$

For the $2^{\text {nd }}$ equality, we have made use of Equations, (5-22), and, (2-25). To 
find the total gravitational force, at a specific radius $r$ within the black hole, we integrate Equation, (5-29). We obtain as a result,

$$
\begin{aligned}
F_{G, r} & =\int_{0}^{r}\left(g_{r}\right)\left(\rho_{r} A_{r} \mathrm{~d} r\right)=\int_{0}^{r}\left(-0.1 c^{2} r / \sigma^{2}\right)\left(M_{R} f_{r} \mathrm{~d} r\right) \\
& =\left(-0.1 M_{R} c^{2}\right) \int_{0}^{r}\left(r / \sigma^{2}\right)\left(f_{r} \mathrm{~d} r\right) \\
& =\left(-0.1 M_{R} c^{2}\right) \int_{0}^{r}\left(r / \sigma^{2}\right)\left(f_{R} \exp \left[\frac{R^{2}-r^{2}}{2 \sigma^{2}}\right] \mathrm{d} r\right) \\
& =\left(-0.1 M_{R} c^{2} f_{R}\right) \int_{0}^{r}\left(r / \sigma^{2}\right)\left(\exp \left[\frac{R^{2}-r^{2}}{2 \sigma^{2}}\right] \mathrm{d} r\right) \\
& =-F_{R}\left[\mathrm{e}^{R^{2} / 2 \sigma^{2}}-\mathrm{e}^{\left(R^{2}-r^{2}\right) / 2 \sigma^{2}}\right]
\end{aligned}
$$

For the $3^{\text {rd }}$ line, we have substituted Equation, (4-21). And for the $5^{\text {th }}$ line, we have used Equation, (3-10), to simplify the constant factor in front of the integral.

We notice that Equation, (5-30), equals zero when $r=0$, as it must. We also notice that, if $r=R$, then we obtain,

$$
F_{G, R}=-F_{R}\left[\mathrm{e}^{R^{2} / 2 \sigma^{2}}-1\right]
$$

Both Equations, (5-30), and (5-31), are rather simple. As always, they are a direct consequence of the pdf chosen. We can use these equations directly to find the 4-D gravitational force as a function of radius. We know, the, $\sigma$, as well as the, $R$, values for the three black holes under consideration. See Equations, (3-20), and, (3-18). We also know the value for the radiative force, $F_{R}=F_{R}^{(4)}$, just inside the event horizon. These are given by Equations, (4-17), for the three black holes. The values for the gravitational force are tabulated under column 11 in each of the Tables 4-6.

In the first paper [5], we spent a considerable of time talking about the significance of the surface tension. In fact, we noted that it consisted of two components, a radiative as well as a gravitational component,

$$
F_{\mathrm{ST}}=\left(F_{\mathrm{ST} \text { radiative }}+F_{G, R}^{(4)}\right)=F_{R}^{(3)}-F_{R}^{(4)}+F_{G, R}^{(4)}
$$

See Equations, (3-10), or, (3-13), in reference [5]. The radiative force, $F_{R}^{(3)}$, is the 3-D radiative force pushing the event horizon in, whereas, $F_{R}^{(4)}$, is the 4-D radiative force pushing the event horizon out. The surface tension is important in determining the work needed in expanding the black hole. Very simply, to increase the radius of a black hole by an amount, $\mathrm{d} R$, from surface radius, $R$, to, $R+\mathrm{d} R$, it takes the following amount of work, $\mathrm{d} W=F_{\mathrm{ST}} \mathrm{d} R$. We compare the radiative surface tension in Equation, (5-32), to the gravitational surface tension within the same equation. From reference [5], we obtained

$$
\begin{aligned}
M_{B H} & =\left(M_{\text {sun }}, 10 M_{\text {sun }}, 10^{6} M_{\text {sun }}\right) \\
\rightarrow F_{\text {ST radiative }} & =\left(3.05 \times 10^{-7}, 3.05 \times 10^{-5}, 3.05 \times 10^{+5}\right)
\end{aligned}
$$


Refer to Equation, (3-19), specifically, in the work cited. These values hold for an isolated, static black hole, where there is virtually no net radiative heat coming in. We compare these values to the gravitational force values, indicated in Tables 4-6. There we obtain, at the surface,

$$
\begin{gathered}
M_{B H}=\left(M_{\text {sun }}, 10 M_{\text {sun }}, 10^{6} M_{\text {sun }}\right) \\
\rightarrow F_{\text {ST gravity }}=F_{G, R}=\left(7.30 \times 10^{43}, 7.16 \times 10^{45}, 6.36 \times 10^{43}\right)
\end{gathered}
$$

In both equations, forces are measured in Newtons. We see that there is no comparison. Radiative forces cannot compete against gravitational forces when expanding a black hole in this situation, where there is virtually no net radiative heat inflow.

If there is substantial net heat inflow, the radiative surface tension contribution may not negligible. Consider an external, outside temperature of, $T_{2}=10^{9} \mathrm{~K}$, which would correspond to hard X-rays, having energies in the 100 $\mathrm{keV}$ range. We can work out the radiative forces, in terms of radius, $R$. We obtain using our, by now, familiar relations,

$$
\begin{gathered}
F_{R}^{(3)}=p_{2}^{(3)} A_{R}^{(3)}=\frac{1}{3} a^{(3)}\left(10^{9}\right)^{4} 4 \pi R^{2}=3.17 \times 10^{21} R^{2} \\
F_{R}^{(4)}=p_{1}^{(4)} A_{R}^{(4)}=\frac{1}{4} a^{(4)}\left(0.0597 \times R^{-1}\right)^{5} 2 \pi^{2} R^{3}=1.40 \times 10^{-13} R^{2}
\end{gathered}
$$

We have used Equations, (4-8a,b), (2-16a), (2-17) and, (4-9a), to obtain the above. The 3-D internal energy density can be expressed as, $u_{R}^{(3)}=a^{(3)} T^{4}=\left(7.5657 \times 10^{-16}\right) T^{4}$, in MKS units, a well-known result. The radiative surface tension is defined as, $F_{\mathrm{ST} \text { radiative }} \equiv F_{R}^{(3)}-F_{R}^{(4)}$. For the three black holes, we therefore find, using the definition for radiative surface tension, and Equations, (5-35), with, (5-36),

$$
\begin{gathered}
M_{B H}=\left(M_{\text {sun }}, 10 M_{\text {sun }}, 10^{6} M_{\text {sun }}\right) \\
\rightarrow F_{\text {ST radiative }}=\left(2.77 \times 10^{28}, 2.77 \times 10^{30}, 2.77 \times 10^{40}\right)
\end{gathered}
$$

We have used the radii listed in Equations, (3-18). These values in Equations, (5-37), are still much less than those indicated by Equation, (5-34). However, for very massive black holes $\left(M_{B H}>10^{6} M_{\text {sun }}\right)$, with external temperatures very high $\left(T_{2}>10^{9} \mathrm{~K}\right)$, we can see that we are approaching a situation where the radiative contributions to surface tension can no longer simply be ignored.

Our final step is step 9. In this step, we evaluate the entropy of a black hole, $S_{r}$, as a function of radius. We treat the entropy as an extrinsic quantity, dependent on volume. Our volume, however, is a 4-dimensional one. In a volume element, $\mathrm{d} V_{r}$, between $r$ and $r+\mathrm{d} r$, we can expect a value for entropy in the amount, $\mathrm{d} S_{r}=s_{r} \mathrm{~d} V_{r}$, where $s_{r}$ is the entropy density in layer, $r$ and $r+\mathrm{d} r$. The entropy up to radius, $r$, can be found by integrating this expression. We find that,

$$
S_{r}=S_{r}^{(4)}=\int_{0}^{r} S_{r} \mathrm{~d} V_{r}=\int_{0}^{r} S_{r} A_{r} \mathrm{~d} r
$$


The, $A_{r}=2 \pi^{2} r^{3}$, is the 4-D surface area at radius, $r$. On the left hand side, we have fixed the constant of integration, $S_{0}=0$, as we believe that there can be no entropy if the volume is zero.

We know, however, that the entropy density can be related to radiative pressure, when multiplied by a volume or surface area. From Equations, (2-18), we can write,

$$
s_{r} A_{r}=5 / 4 u_{r} A_{r} / T_{r}=5 p_{r} A_{r} / T_{r}
$$

This expression will be substituted into Equation, (5-29), and we obtain,

$$
S_{r}=\int_{0}^{r} S_{r} A_{r} \mathrm{~d} r=\int_{0}^{r} 5 p_{r} A_{r} / T_{r} \mathrm{~d} r=5 \int_{0}^{r} F_{r} / T_{r} \mathrm{~d} r
$$

For the last equality, we employed Equation, (2-23), which relates radiative force to radiative pressure.

Next, we utilize, Equation, (4-23). Substituting this for, $F_{r}$, allows us to re-express Equation, (5-40), as follows.

$$
S_{r}=5 \int_{0}^{r} F_{r} / T_{r} \mathrm{~d} r=5 F_{R} \int_{0}^{r} \exp \left[\left(R^{2}-r^{2}\right) / 2 \sigma^{2}\right] / T_{r} \mathrm{~d} r
$$

We will also make use of relation, (4-25), which we will rewrite as,

$$
1 / T_{r}=1 / T_{R}(r / R)^{3 / 5} \exp \left[-\left(R^{2}-r^{2}\right) / 10 \sigma^{2}\right]
$$

We substitute this Equation, (5-42), into the integrand of Equation, (5-41), in order to eliminate the $1 / T_{r}$ term. Upon simplifying, we find that

$$
S_{r}=\left(5 F_{R} / T_{R}\right)\left(1 / R^{3 / 5}\right) \int_{0}^{r} r^{3 / 5} \exp \left[0.4\left(R^{2}-r^{2}\right) / \sigma^{2}\right] \mathrm{d} r
$$

This is an expression, which can be integrated. The result is

$$
S_{r}=\left(-F_{R} / T_{R}\right) R^{-\frac{3}{5}}(5 / 2)^{\frac{9}{5}}\left[\Gamma\left(\frac{4}{5}, \frac{2 r^{2}}{5 \sigma^{2}}\right)-\Gamma\left(\frac{4}{5}\right)\right] \sigma^{8 / 5} \mathrm{e}^{\frac{2 R^{2}}{5 \sigma^{2}}}
$$

In this equation, $\Gamma(s)$, is the gamma function and, $\Gamma(s, x)$, is the upper incomplete gamma function, defined in the Appendix. Using the identities given in the Appendix, we can re-express Equation, (5-44), in terms of the lower incomplete gamma function, $\gamma(s, x)$. The result is,

$$
S_{r}=\left(+F_{R} / T_{R}\right) R^{-\frac{3}{5}}(5 / 2)^{\frac{9}{5}} \gamma\left(\frac{4}{5}, \frac{2 r^{2}}{5 \sigma^{2}}\right) \sigma^{8 / 5} \mathrm{e}^{\frac{2 R^{2}}{5 \sigma^{2}}}
$$

This gives the entropy as a function of radius within the black hole. In hindsight, Equation, (5-45), could have been obtained by using Equation, (4-27). This would have been a little more direct, but then we would not have seen the connection between temperature and radius, as specified in Equation, (5-42). And the quantity, $s_{R}$, would also have to be worked out.

The lower incomplete gamma function, $\Gamma(s, x)$, in Equation, (5-45), can be evaluated for various radii within the black hole since we know the $\sigma$ value. We also know the radiative force, $F_{R}$, just inside the black hole, as well as its radius. Finally, the temperature, $T_{R}=T_{1}$, is also known. Therefore, the entropy, 
$S_{r}$, can be calculated for each of the black holes considered. The results are presented under column 12 in the Tables 4-6. For the various radii, Equations, (3-18), were utilized. For the shape parameters, Equations, (3-20), were employed. The corresponding 4-D inside surface temperatures were specified by Equations, (4-10). And in relations, (4-17), we have the respective radiative forces, just inside the event horizons for the black holes being analyzed. All the quantities in Equation, (5-36), are 4-D quantities.

We note that because of the mathematical inequality, $\Gamma(s)>\Gamma(s, x)$, the entropy, as given by expression, (5-35), will always be positive. We also give the Excel function for the lower incomplete gamma function, as this is somewhat unusual. To calculate, $\gamma(s, x)$, we may use

$$
\gamma(s, x)=\operatorname{EXP}(\operatorname{GAMMALN}(s)) * \operatorname{GAMMA} \cdot \operatorname{DIST}(x, s, 1, T R U E)
$$

Utilizing this expression, one can verify these results.

We are now in a position to discuss our results. We first notice upon inspection of Tables 4-6, that the radiative mass in each instance is very much concentrated within the core of the black holes. In fact, within a radius of $15 \%$ of the total radius, we have the following percentages of total mass contained, for the three black holes being considered:

$$
\left(M_{r=0.15 R}^{A}, M_{r=0.15 R}^{B}, M_{r=0.15 R}^{C}\right)=\left(97.7 \% M_{R}^{A}, 97.4 \% M_{R}^{B}, 95.2 \% M_{R}^{C}\right)
$$

These percentages indicate that the radiative mass is very much localized within a relatively small core volume. The cumulative probability distribution function, $F_{r}^{C D F}$, listed under column 2, represents the actual percentage of total mass contained within a given radius. See Equation, (2-11).

The same percentages hold for the total radiative energy, $E_{r=0.15 R}$, at $r=0.15 R$, the internal energy, $U_{r=0.15 R}$, at $r=0.15 R$, and the heat energy, $Q_{r=0.15 R}$, at $r=0.15 R$. All these quantities are proportional to, $M_{r=0.15 R}$, as can be seen by Equations, (4-22), and, (4-23). Once we know how radiative mass is distributed within the black hole, we effectively know how energy, in all its forms, is likewise distributed. We have chosen a specific pdf to model the mass distribution within the interior of the black hole. This fixes column 2 in all these tables. And from column 2, we derive the other quantities. Again, we can think of the black hole as an exotic type of capacitor in that it can store radiative energy. How specifically that energy is packed will depend on the pdf, which is chosen.

A third realization is that gravity varies within the black hole. Surprisingly, it is radius dependent, a consequence of the pdf chosen. Thus, in 4-D space, the 4-D gravitational "constant", $G_{r}=G_{r}^{(4)}$, is not a constant. Also at the event horizon, its value changes abruptly. Upon entering the black hole from 3-D space, its value increases dramatically from, $G=G_{N}$, which is Newton's constant, to $G_{R}=G_{R}^{(4)}$. We can set up a ratio of $G_{R} / G$. This ratio is not dimensionless as the gravitational "constant" in 4-D space has units of, Newtons $\mathrm{m}^{3} / \mathrm{kg}^{2}$. We find for the three black holes under investigation, the following increases upon en- 
tering the black hole form 3-D space:

$$
\left(G_{R}^{A} / G, G_{R}^{B} / G, G_{R}^{C} / G\right)=\left(1.35 \times 10^{5}, 1.30 \times 10^{6}, 1.03 \times 10^{11}\right) \text { meters }
$$

The larger the black hole, the greater the increase upon entering 4-D space. For supermassive black holes, this increase is particularly impressive.

A fourth surprise is entropy. Contrary to current thinking, the entropy in our model suggests that a black hole may be a highly ordered state. Our entropy value, in relative terms, is small, very much smaller than the Bekenstein result. Also, our entropy scales with increasing radius, comparable to $R^{2}$, but not with a clean power law. Our expression for entropy is given by Equation, (5-45). At radius, $r=R$, which characterizes the event horizon, this quantity has been calculated in each of the tables. For the three black holes being analyzed, we find

$$
\left(S_{R}^{A}, S_{R}^{B}, S_{R}^{C}\right)=\left(1.63 \times 10^{37}, 6.59 \times 10^{40}, 7.24 \times 10^{46}\right)
$$

The units for 4-D entropy are, Joule/K, in the MKS system. According to Bekenstein, the sun has an entropy of roughly, $10^{35} \mathrm{~J} / \mathrm{K}$. For a black hole having the mass of the sun, he calculates a value equal to, $1.5 \times 10^{54} \mathrm{~J} / \mathrm{K}$. Our value for a black hole having the mass of the sun is different, $1.63 \times 10^{37} \mathrm{~J} / \mathrm{K}$, which is about one hundred times larger than that of the sun, but much, much lower than his estimate. See the first equality in Equations, (5-49). Moreover, his entropy scales as, $S_{\text {Bekenstein }} \sim R^{2}$. Our entropy also scales, but the scaling behavior is complicated. Upon inspection of the values indicated by Equations, (5-49), there is much variation between the various masses, and corresponding radii. If our result is to be believed, most of the entropy in the universe is, thus, not in the form of black holes. As with other calculated global quantities, it needs to be emphasized that our formula for entropy depended on our choice for distribution function. If we had chosen a different pdf, other than a truncated Gaussian, the results for entropy would be entirely different.

As with radiative mass, most of the entropy, is to be found within the very interior of the black hole. Resorting to the tables, we can calculate how much entropy we have, as a percentage of the total, at a radius of, $r=0.15 R$. We find,

$$
\left(S_{r=0.15 R}^{A}, S_{r=0.15 R}^{B}, S_{r=0.15 R}^{C}\right)=\left(91.2 \% S_{R}^{A}, 90.4 \% S_{R}^{B}, 84.9 \% S_{R}^{C}\right)
$$

We see that we have accounted for $85 \%+$ of the total entropy, within an inner radius, $15 \%$ of the total.

\section{Summary and Conclusions}

In this work, we analyzed what the internal structure of a black hole could look like, given our 4-D blackbody radiation model for a black hole. We treat the black hole as a self-contained, spherically symmetric, steady state, 4-D spatial ball filled with radiation. The blackbody radiation produces the radiative mass and we chose a particular pdf, the truncated Gaussian, to pack the mass a certain way within its interior. Any truncated pdf with support, $r \in[0, R]$, hypothetically, could have been chosen, but the truncated Gaussian is relatively simple, 
somewhat familiar, and leads to acceptable results. We fixed the parameters by assuming that we have maximum radiative energy at the center, which allowed us to set, $\mu=0$. We were also able to determine the shape parameter, $\sigma$, by using the temperature just inside the event horizon. This inner surface temperature was calculated using a generalized version of the Stefan-Boltzmann law, derived in a previous paper, and which allows for radiative transfers between spaces having different dimensions. In a very real sense, we can think of a black hole as an exotic type of capacitor, but one which stores energy in 4-D space, in a non-uniform way, and, in blackbody form. The distribution of radiative energy is not constant; rather it follows a pdf, which has to be specified and fulfill certain conditions. We treat the black hole as a 4-D construct, for reasons given in a previous work. If our model is correct, the rip or tear in the space-time continuum occurs not at the center of the black hole, but rather at its surface, because it is there that we are transitioning between 3-D and 4-D space. The event horizon is assumed infinitely thin.

In Section 2, we considered two stellar-like equations, conservation of mass and hydrostatic equilibrium. We generalized these equations to 4 -D space. We discovered that the radiative mass density cannot be uniformly distributed. First, the temperature at the surface of the black hole, on the inside, would be too high, if we want to accommodate the required mass. Second, and perhaps more importantly, we would have no gravitational forces within its interior. For that to happen, a temperature gradient is necessary. The only gravity which would then exist would be at the surface. We therefore introduced a generalized Gaussian distribution, but truncated so that only values of radii within the black hole are admissible. The 4-D generalization of the hydrostatic equilibrium Equation, (2-14), was also considered. We found that this had to be modified because it is not just pressure which is changing when one changes the radius. We also have to change the surface area upon which this pressure is acting. Our modification to this equation is relation, (2-30). In Section 2, we argued that the internal energy density, the heat density and the radiative pressure all contributed towards the total energy/radiative mass density. A temperature change will carry over to all these variables, and not selectively to a subset. All these variables are defined in terms of a specific temperature, and if the temperature changes, so, too, do all these variables change, simultaneously.

In Section 3, we looked at the specifics of the truncated Gaussian distribution. We specified the pdf, and the corresponding cdf, for the case where the mode is assumed to be at the center, $r=0$. This fixed our first parameter, $\mu$, associated with this distribution. In this case where we have maximum radiative mass density at the center, $\mu=0$. The second parameter, $\sigma$, called the shape parameter, was determined using the inside surface temperature of the black hole. We determined three values for sigma, for three specific black holes. The results are given by Equations, (3-20). The black hole masses considered were highlighted in Equations, (2-18). For this choice of distribution, Equations, (3-6), 
and, (3-8), tell us how the radiative mass is distributed (packed) within the black hole. In this paper, we considered, by and large, only black holes for which there is no radiative heat inflow. This was to keep the discussion simple. We did indicate, however, how to generalize these results for the situation where there is net heat inflow. Equations, (2-14), (2-15), and, (2-16) apply if there is no net heat inflow. This allowed us to find the pdf value, $f_{R}$, at $r=R$. We then used Equation, (2-18), to fix the $\sigma$ value for each black hole having a different mass, or alternatively, radius.

Section 4 concerned itself with determining the temperature profile, the various densities, the radiative pressures, and the radiative forces within the black hole. All these quantities are functions of the 4-D radius, $r$. After a review of the generalized Stefan-Boltzmann law for a 3-D/4-D interface, we found, $T_{1}$, which is the temperature just inside the event horizon, on the 4-D side. For the three black holes considered in this paper, these temperatures were evaluated. They are given by Equations, (4-10). The calculated temperatures, specified by Equations, (4-10), are much higher than the corresponding Hawking temperatures, but then again, they are defined within a different spatial dimension, and are obtained by different means. The three black holes we focused on, numerically, were those having masses, and radii, as specified under Equations, (3-18).

Once we have the temperatures at the surface, we can use our probability distribution function, to determine the temperature profiles, $T_{r}=T(r)$ within the interior. This will also allow us to calculate, within the interior, the internal energy density, $u_{r}=u(r)$, the radiative pressure, $p_{r}=p(r)$ the heat density, $q_{r}=q(r)$, the total radiative mass density, $\rho_{r}=\rho(r)$, the entropy density, $s_{r}=s(r)$ and the radiative force, $F_{r}=F(r)$. We presented our results in table form, one for each black hole under consideration. Table 1, our first table, gave the results for black hole, $B H_{A}$, which has a mass equal to that of the sun. Table 2 , holds for a black hole, $B H_{B}$, which has a mass, which is ten times that of the sun. And, Table 3 corresponds to a supermassive black hole, $B H_{C}$, which is a million times more massive than that of the sun. The equations that we used to calculate these values were given in the text.

In Section 5, we determined some other attributes associated with the three black holes, which were under investigation. Specifically, we focused on evaluating the total enclosed radiative mass, $M_{r}=M(r)$, up to and including radius, $r$. We also looked at the enclosed radiative internal energy, $U_{r}=U(r)$, the trapped heat, $Q_{r}=Q(r)$ and the work done against radiative pressure, $W_{r}=W(r)$, up to, and including, radius, $r$. These were given by Equations, (5-4), (5-5), and (5-6), respectively. The total radiative energy, $E_{r}=E(r)$, is the sum of these three components, and this is what produces the radiative mass in our model. For their individual evaluation, integration over 4-D space was necessary. Their values, as well as those for, $M_{r}$, are listed in Tables 4-6, for various radii within the black holes. Table 4 holds for black hole, $B H_{A}$, which has one solar mass. Table 5 and Table 6, apply for black holes, $B H_{B}$, and, $B H_{C}$, respectively, 
where black hole, $B H_{B}$, has a mass ten times that of the sun. And black hole, $B H_{C}$, refers to a black hole which is a million times more massive than the sun. We saw that these values were essentially proportional to the cumulative distribution function, $F_{r}^{C D F}$, which is indicated under column 2 in all these tables. Most of the radiative mass $(85 \%+)$, and therefore energy, is to be found within a core radius that is $15 \%$ of the total radius.

In this section, we also evaluated/calculated the gravitational acceleration, $g_{r}=g_{r}^{(4)}$, the gravitational strength, $G_{r}=G_{r}^{(4)}$, the gravitational force, $F_{G, r}=F_{G, r}^{(4)}$, and the gravitational potential, $\varphi_{r}=\varphi(r)$, within the black hole, as functions of radius, $r$. All these quantities were given by relatively simple expressions, a result of the pdf chosen, and they are also listed in the tables indicated above. We saw that upon entering the 4-D black hole, the gravitational "constant" is no longer a constant, but varies with respect to radius. Not only that, but its value increases abruptly upon entering $4-\mathrm{D}$ space. The increases in gravitational "constant" over the Newtonian value is given by Equations, (5-48), which are the increases, for the three black holes under consideration.

Finally, in this section, we evaluated the entropy, $S_{r}=S(r)$, from, $r=0$, up to and including radius, $r$. This was given by a rather complicated function, Equation, (5-45), which involves a lower incomplete gamma function. This result is a consequence of the pdf chosen, a truncated Normal distribution. From Equation, (5-45), we notice that the entropy depends on the full radius, $R$, the inside surface temperature of the black hole, $T_{R}=T_{1}$, the radiative force just inside the event horizon, $F_{R}=p_{1} A_{R}$, and the shape parameter, $\sigma$. We have each of these quantities for the three black holes being analyzed. Thus, we can tabulate the entropy, $S_{r}$, as a function of radius. The results are presented in each of the Tables $4-6$, under column 12 . The total entropy, $S_{R}$, at radius, $R$, is especially interesting. We find that it is very much less than the corresponding Bekenstein result, especially for very large black holes. See Equations, (5-49), where specific values are calculated. Our results also scale with the radius of the black hole, but in a somewhat complicated manner. See Equation, (5-45), where the $R$ dependency is indicated. According to Bekenstein, the entropy should scale as, $S_{\text {Bekenstein }} \sim R^{2}$, making it an intrinsic variable in 3-D space. In sharp contrast to current thinking, our conclusion is that the black hole may be a highly ordered state, if our model is to be taken seriously. Moreover, in Equations, (5-50), we observe that the entropy is pretty much determined within a radius, which is $15 \%$ of the full radius. Almost the entire entropy is contained within the core.

Our model suggests that black holes are, in fact, highly ordered states. In this context, it has been noted that black holes may be well-defined quantum mechanical systems, having ordered discrete quantum spectra [23] [24] [25]. Within this framework, black holes consist of quasi-normal modes (QNM's), which are to be considered normal quantum levels within the black hole. The external structure of a black hole can thus be approximated as a quantized system analogous to the excited electron states within the Bohr model. As a consequence, 
evaporative emissions in the form of Hawking radiation would consist of pure states. This interesting model supports the notion that black holes are, in reality, highly ordered quantized states in a semi-classical approximation. We considered here in this paper a classical picture for black holes, but a quantum mechanical treatment would certainly be an interesting extension of this work.

We focused in this paper on one pdf, and one scenario, an isolated, static black hole, with no heat inflow. Obvious extensions would be to consider net radiative heat inflows, where $\mathrm{d} Q / \mathrm{d} t>0$. There, we would have to specify the specific amount of temperature on the outside of the event horizon. Another extension would be to consider other truncated pdf's with the same support, $r \in[0, R]$, to model the interior radiative mass distribution inside the black hole. These may have more favorable attributes or characteristics. Our goal in this work was to develop a framework, for considering what could happen within the interior of a black hole. It was not, obviously, to play out all scenarios.

Another generalization of this work would be to add other forms of radiation, not just blackbody, and consider their effect within the interior of the black hole. We could also incorporate massive particles, obeying Fermi-Dirac statistics. If the temperature is above the so-called "freeze-out" temperature, these particles are in the form of radiation. Below the freeze-out temperature, we expect distinct massive particles. We have seen that the temperature within the black hole assume a distribution of temperatures. Thus, there could be regions within the black hole where we have particles, and other regions, where we have radiations. For a one solar mass black hole (see Table 1), the temperature decreases from, $T=4.14 \times 10^{12} \mathrm{~K}$, at a radius, $r=10^{-6} R$, down to, $T=1.09 \times 10^{9} \mathrm{~K}$, at a radius, $r=0.2 R$. These are high temperatures, which correspond to energies of, 357 $\mathrm{MeV}$, down to, $94 \mathrm{keV}$, respectively. Above, $214 \mathrm{MeV}$, most of the particles in the standard model [1] [2] [3] [4] are in thermodynamic equilibrium with the blackbody photons. To take into account both fermionic and bosonic relativistic species, we must specify a scale factor, $g_{\text {TотАL }}^{*}(T)$, which is temperature dependent. At about, $214 \mathrm{MeV}$, the scale factor, $g_{\text {TотаL }}^{*}=62.5$. We can multiply the internal energy density of blackbody photons by a factor, such as this, to estimate what the black hole is capable of holding energy-wise, if we wish to take into account other forms of radiation. This is another area for further study.

Above background energies of, $1 \mathrm{GeV}$, which corresponds to a temperature of, $1.16 \times 10^{13} \mathrm{~K}$, the scale factor becomes, $g_{\text {TОтАL }}^{*}=75.5$. And at background energies of approximately, $1 \mathrm{TeV}$, which corresponds to a temperature of, $1.16 \times 10^{16}$ $\mathrm{K}$, the scale factor increases to, $g_{\text {TOTAL }}^{*}=106.75$. Above, $1 \mathrm{TeV}$, all particles in the standard model are relativistic. Interestingly such temperatures are only exceeded for incredibly small black holes. We saw that the smaller the black hole, the greater the temperature within its very core. As mentioned in the text, in passing, this may have ramifications for particle physics, and the modeling of elementary particles as mini black holes. This is a further area of investigation. One can consider the modeling of elementary particles as mini-black holes.

It is difficult to see, at present, how this model can be tested observationally. 
We can estimate outside surface temperatures by the electromagnetic emissions being given off next to a black hole. We can also estimate the black hole mass, and size, by the trajectory of stars in its vicinity. This should allow us to estimate inside surface temperature. Net heat flow can then be calculated using our generalized Stefan-Boltzmann equation. We have assumed that the heat flowing in is a direct function of the surface temperature on the outside, versus that on the inside. For a temperature on the inside, we assumed a-priori, that, for a specific radius, it can be calculated as if the black hole were an isolated, static black hole. This is a working assumption, which may or may not hold true. With all these assumptions, we might observe how a black hole expands with time, and see if our relations make sense in predicting radiative heat inflow with corresponding expansion. We believe that 4-D space is probably beyond our observational reach. Probing this space to verify the radial properties within the black hole may well prove to be the ultimate challenge.

Finally, relating to probing 4-D space, Hawking radiation could be considered. Hawking radiation was largely ignored in this work as it was argued that evaporative processes were $2^{\text {nd }}$ order effects. Our inside surface black hole temperatures were shown to be much higher than the corresponding Hawking temperatures, and thus evaporative processes were largely ignored/discounted. We calculated and compared temperatures in the text for various massive black holes. We also showed that the inside temperature is much, much smaller than the outside 3-D temperatures, with or without radiative inflow. (In brane theory, one has a similar result... the temperatures are higher in higher dimensional space.) However, Hawking radiation and evaporative processes should be looked at, and studied, more carefully in light of the peculiarities of our 4-D spatial model. If the inside surface temperatures are higher, this actually may have important ramifications for leakage.

As is known [26], the spectrum of particle emissions in terms of energy flux is dependent on the type of black hole considered as well as type of particle emitted. For example, a non-rotating black hole emits spin zero particles pretty much like a perfect blackbody spectrum. A rotating black hole, on the other hand, emits a series of peaks, which is dependent on the rotation parameter and angular velocity. See, for example, Figure 3-3 and Figure 3-4, versus Figure 3-5, in the reference cited, where the latter figure refers to a spinning black hole. Furthermore, qualitatively, higher spin particles follow the same pattern. By considering evaporative processes, one might conceivably infer what is happing within the 4-D black hole. This is obviously a further line of inquiry, and research, which can, and should be pursued.

\section{Acknowledgements}

With these two papers, the author would like to thank his children, Kira, Juergen, and Henry, for their love and support. They have been a constant source of encouragement, inspiration, joy and goodwill, throughout my entire life. They are, 
first and foremost, my proudest achievement, and will always remain so.

\section{Conflicts of Interest}

The author declares no conflicts of interest regarding the publication of this paper.

\section{References}

[1] Kolb, E.W. and Turner, M.S. (1989) The Early Universe. Addison-Wesley, Reading.

[2] Baumann, D.D. (2015) Lecture Notes on Cosmology. http://theory.uchicago.edu/ liantaow/my-teaching/dark-matter-472/lectures.pdf

[3] Mather, J.C., et al. (1999) Calibrator Design for the COBE Far-Infrared Absolute Spectrophotometer (FIRAS). The Astrophysical Journal, 512, 511-520. https://doi.org/10.1086/306805

[4] Husdal, L. (2016) On Effective Degrees of Freedom in the Early Universe. Galaxies, 4, 78. https://doi.org/10.3390/galaxies4040078

[5] Christopher, P. (2019) Are Black Holes 4-D Spatial Spheres Filled with Black Body Radiation? Generalization of the Stefan-Boltzmann Law and Young-Laplace Relation for Spatial Radiative Transfers. Journal of High Energy Physics, Gravitation, and Cosmology, 5, 638-682. https://doi.org/10.4236/jhepgc.2019.53036

[6] Zeilik, M.A. and Gregory, S.A. (1998) Introductory Astronomy \& Astrophysics. 4th Edition, Saunders College Publishing, Philadelphia.

[7] Hansen, C.J., Kawaler, S.D. and Trimble, V. (2004) Stellar Interiors. 2nd Edition, Springer, Berlin.

[8] Landsberg, P.T. and De Vos, A. (1989) The Stefan Boltzmann Constant in an N-Dimensional Space. Journal of Physics A: Mathematics and General, 22, 1073-1084. https://doi.org/10.1088/0305-4470/22/8/021

[9] Menon, V.J. and Agrawal, D.C. (1998) Comment on "The Stefan-Boltzmann Constant in N-Dimensional Space". Journal of Physics A: Mathematics and General, 31, 1109-1110. https://doi.org/10.1088/0305-4470/31/3/021

[10] Barrow, J.D. and Hawthorne, W.S. (1990) Equilibrium Matter Fields in the Early Universe. Monthly Notices of the Royal Astronomical Society, 243, 608-609.

[11] Gonzalez-Ayala, J., Perez-Oregon, J., Cordero, R. and Angulo-Brown, F. (2015) A Possible Cosmological Application of Some Thermodynamic Properties of the Black Body Radiation in N-Dimensional Euclidean Spaces. Entropy, 17, 4563-4581. https://doi.org/10.3390/e17074563

[12] Gonzalez-Ayala, J., Cordero, R. and Angulo-Brown, F. (2016) Is the (3+1)-d Nature of the Universe a Thermodynamic Necessity? EPL (Europhysics Letters), 113, 4006. https://doi.org/10.1209/0295-5075/113/40006

[13] Johnson, N.L., Kotz, S. and Balakrishnan, N. (1994) Continuous Univariate Distributions. Volume 1, Wiley, Hoboken.

[14] Johnson, N.L. and Kotz, S. (1970) Continuous Univariate Distributions-1. Chapter 13, John Wiley \& Sons, Hoboken.

[15] Barr, D.R. and Sherrill, E.T. (1999) Mean and Variance of Truncated Normal Distributions. The American Statistician, 53, 357-361. https://doi.org/10.1080/00031305.1999.10474490

[16] Burkardt, J. (2014) The Truncated Normal Distribution. Department of Scientific 
Computing Website, Florida State University, Tallahassee.

[17] Lecture 4: Selection. web.ist.utl.pt. Instituto Superior Técnico, Lisboa, 1.

[18] Fernandez-de-Cossio-Diaz, J. (2017) Truncated Normal: Compute Mean and Variance of the Univariate Truncated Normal Distribution (Works Far from the Peak).

[19] Johnson, N.L., Kotz, S. and Balakrishnan, N. (1995) Beta Distributions. In: Johnson, N.L., Kotz, S. and Balakrishnan, N., Eds., Continuous Univariate Distributions, Vol. 2, 2nd Edition, Wiley, Hoboken, Chapter 21.

[20] Jones, M.C. (2009) Kumaraswamy's Distribution: A Beta-Type Distribution with Some Tractability Advantages. Statistical Methodology, 6, 70-81. https://doi.org/10.1016/j.stamet.2008.04.001

[21] Forge Project. Handbook on Probability Distributions: R-Forge Distributions Core Team University Year 2009-2010.

https://r-forge.r-project.org/scm/viewvc.php/* checkout*/pkg/inst/doc/probdistr-ma in.pdf? revision $=24 \&$ root $=$ distributions \&pathrev $=24$

[22] Another Useful Handbook of Probability Distribution Functions. http://www.stat.rice.edu/ dobelman/textfiles/DistributionsHandbook.pdf

[23] Corda, C., Hendi, S.H., Katebi, R. and Schmidt, N.O. (2013) Bohr-Like Black Holes. Journal of High Energy Physics, 06, 008.

[24] Corda, C. (2015) Time Dependent Schroedinger Equation for Black Hole Evaporation; No Information Loss. Annals of Physics, 353, 71-82. https://doi.org/10.1016/j.aop.2014.11.002

[25] Corda, C. (2015) Precise Model of Hawking Radiation from the Tunneling Mechanism. Classical and Quantum Gravity, 32, Article ID: 195007.

https://doi.org/10.1088/0264-9381/32/19/195007

[26] Calmet, X., Carr, B. and Winstanley, E. (2014) Quantum Black Holes. Springer Briefs in Physics, Springer Verlag, New York. 


\section{Appendix}

Here, we present certain formulae, as it relates to the truncated Gaussian distribution, and identities relating to the calculation of the entropy. These are given for reference purposes. The symbols are explained in the text.

The truncated Gaussian distribution function is given by,

$$
f_{r}=f(r, 0, \sigma ; 0, R)=\sqrt{(2 / \pi)} 1 / \sigma \exp \left(-r^{2} /\left(2 \sigma^{2}\right)\right) 1 / \operatorname{erf}(R /(\sqrt{2} \sigma))
$$

The corresponding cumulative distribution function reads,

$$
F_{r}^{C D F}=F(r, 0, \sigma ; 0, R)=\operatorname{erf}(r / \sqrt{2} \sigma) / \operatorname{erf}(R / \sqrt{2} \sigma)
$$

We have assumed that the radius has support, $r \in[a, b]=[0, R]$, and that the mode equals zero. Thus, $\mu=0$. The shape parameter, $\sigma$, is determined by the inside surface radiative force, $F_{R}=F_{R}^{(4)}$, within the 4-D black hole. The function, $\operatorname{erf}(x)$, is the error function.

$$
\begin{gathered}
\sqrt{(\pi / 2)} \sigma \mathrm{e}^{R^{2} /\left(2 \sigma^{2}\right)} \operatorname{erf}(r / \sqrt{2} \sigma)=\mathrm{e}^{\left(R^{2}-r^{2}\right) /\left(2 \sigma^{2}\right)} F_{r}^{C D F} / f_{r} \\
F_{R}^{(4)} / f_{R}=p_{1}^{(4)} A_{R}^{(4)} /\left(\rho_{1}^{(4)} A_{R}^{(4)}\right) M_{R}=0.1 M_{R} c^{2} \\
F_{r}^{(4)} / f_{r}=p_{r}^{(4)} A_{r}^{(4)} /\left(\rho_{r}^{(4)} A_{r}^{(4)}\right) M_{R}=0.1 e_{r} / e_{r} M_{R} c^{2}=0.1 M_{R} c^{2} \\
\int_{0}^{r} \mathrm{e}^{\left(R^{2}-r^{2}\right) /\left(2 \sigma^{2}\right)} \mathrm{d} r=\int_{0}^{r} f_{r} / f_{R} \mathrm{~d} r=1 / f_{R} \int_{0}^{r} f_{r} \mathrm{~d} r=F_{r}^{C D F} / f_{R} \\
F_{r}^{C D F} / f_{R}=M_{R} F_{r}^{C D F} /\left(M_{R} f_{R}\right)=M_{r} /\left(\rho_{R} A_{R}\right)=M_{r} c^{2} /\left(e_{R} A_{R}\right)=0.1 M_{r} c^{2} / F_{R}
\end{gathered}
$$

Some identities relating to the calculation of entropy follow. Let,

$$
\begin{gathered}
\gamma(s, x)=\text { lower incomplete gamma function }=\int_{0}^{x} t^{s-1} \mathrm{e}^{-t} \mathrm{~d} t \\
\Gamma(s, x)=\text { upper incomplete gamma function }=\int_{x}^{\infty} t^{s-1} \mathrm{e}^{-t} \mathrm{~d} t
\end{gathered}
$$

Then,

$$
\gamma(s, x)+\Gamma(s, x)=\Gamma(s)
$$

where, $\Gamma(s)$, is the gamma function. From this, it follows that

$$
\gamma(s, x)=\Gamma(s)-\Gamma(s, x)>0
$$

This inequality will always lead to a positive value for entropy.

$$
\begin{aligned}
\int_{0}^{r} \mathrm{e}^{0.4\left(R^{2}-r^{2}\right) / \sigma^{2}} r^{3 / 5} \mathrm{~d} r & =-\frac{5^{\frac{4}{5}}}{2^{\frac{9}{5}}}\left[\Gamma\left(\frac{4}{5}, \frac{2 r^{2}}{5 \sigma^{2}}\right)-\Gamma\left(\frac{4}{5}\right)\right] \sigma^{8 / 5} \mathrm{e}^{0.4 R^{2} / \sigma^{2}} \\
& =+\frac{5^{\frac{4}{5}}}{2^{\frac{9}{5}}} \gamma\left(\frac{4}{5}, \frac{2 r^{2}}{5 \sigma^{2}}\right) \sigma^{8 / 5} \mathrm{e}^{0.4 R^{2} / \sigma^{2}}
\end{aligned}
$$

The symbol, $r$, refers to the 4 -D radius within the black hole. Other identities are derived in the text. 